\title{
Terthiophene Radical Cations End-Capped by Bicyclo[2.2.2]octene Units: Formation of Bent $\pi$-Dimers Mutually Attracted at the Central Position
}

\author{
Daisuke Yamazaki, Nobuhide Tanino, Tohru Nishinaga, and Koichi Komatsu* \\ Institute for Chemical Research, Kyoto University, Uji, Kyoto 611-0011, Japan, and Department \\ of Chemistry, Graduate School of Science, Tokyo Metropolitan University, Hachioji, Tokyo \\ 192-0397, Japan
}

\section{Contents}

Experimental

4,5:4",5"-Bis(bicyclo[2.2.2] octeno)-2,2':5',2"'-terthiophene (2)

Hexafluoroantimonate Salt of Radical-Cation of Terthiophene $2\left(\mathbf{2}^{\cdot+} \mathrm{SbF}_{6}{ }^{-}\right)$.

Figure S1. ${ }^{1} \mathrm{H}$ NMR of 2

Figure S2. ${ }^{13} \mathrm{C}$ NMR of 2

X-Ray crystallography

Ortep drawing (50\% probability ellipsoids) and crystal data of $\mathbf{2}$

Ortep drawing (50\% probability ellipsoids), packing structure and crystal data of $\mathbf{2}^{\cdot+} \mathbf{S b F}_{\mathbf{6}}{ }^{-}$at $100 \mathrm{~K}$ 


\section{General}

${ }^{1} \mathrm{H}(300 \mathrm{MHz})$ and ${ }^{13} \mathrm{C}(75.4 \mathrm{MHz}) \mathrm{NMR}$ spectra were recorded on a Varian Mercury-300 spectrometer. Chemical shifts are reported in $\delta \mathrm{ppm}$ from TMS using the signals of the solvent $\left(\mathrm{CDCl}_{3} ; \delta 7.26\right.$ in ${ }^{1} \mathrm{H}\left(\mathrm{CHCl}_{3}\right)$ and $\delta 77.0$ in ${ }^{13} \mathrm{C} \mathrm{NMR}$, respectively) as internal references. Mass spectra (EI) were recorded on a JEOL JMS-HX110 or JMS700 spectrometer. ESR spectra were taken on a Bruker EMX spectrometer. Separation by gel-permeation chromatography (GPC) was performed with a JAI LC-908 chromatograph equipped with JAIGEL $1 \mathrm{H}$ and $2 \mathrm{H}$ columns. Cyclic voltammetry was performed on a BAS-50W electrochemical analyzer. The CV cell consisted of a glassy carbon working electrode, a Pt wire counter electrode, and an $\mathrm{Ag} / \mathrm{AgNO}_{3}$ reference electrode. The measurements were carried out with $1.0 \mathrm{mM}$ solutions of substrate using tetrabutylammonium perchlorate as a supporting electrolyte $(0.1 \mathrm{M})$, and the values of oxidation potential were calibrated using ferrocene as an internal standard.

All reactions were conducted under a dry argon atmosphere unless otherwise stated. THF was distilled from sodium benzophenone ketyl. Dichloromethane, hexane, carbon tetrachloride, and carbon disulfide were distilled over $\mathrm{CaH}_{2}$. The reagents were of the reagent grade obtained commercially unless otherwise stated.

4,5:4",5"-Bis(bicyclo[2.2.2] ]cteno)-2,2':5',2"-terthiophene (2). In a $50 \mathrm{~mL}$ flask, a mixture of 2-(trimethylstannyl)-4,5-bicyclo[2.2.2] octenothiophene $^{1}(0.640 \mathrm{~g}, 1.96 \mathrm{mmol})$, 2,5-dibromothiophene (0.215 g, $0.887 \mathrm{mmol}), \mathrm{Pd}\left(\mathrm{PPh}_{3}\right)_{4}(0.114 \mathrm{~g}, 0.0982 \mathrm{mmol})$ and $\mathrm{CuO}(0.156$ $\mathrm{g}, 1.96 \mathrm{mmol})$ in $20 \mathrm{~mL}$ of dry DMF was degassed with a vacuum pump for 1 minute and the reaction vessel was refilled with argon. The mixture was stirred at $100{ }^{\circ} \mathrm{C}$ for $5 \mathrm{~h}$ and then cooled to room temperature. To the mixture was added $50 \mathrm{~mL}$ of $\mathrm{CH}_{2} \mathrm{Cl}_{2}$ and the precipitates were filtered off. Removal of the volatiles of the filtrate under reduced pressure and GPC 
separation of the residue gave $0.350 \mathrm{~g}$ of $2(96 \%)$ as a yellow solid: $\mathrm{mp} 263.2-264.1{ }^{\circ} \mathrm{C}$; UV-vis $\left(\mathrm{CH}_{2} \mathrm{Cl}_{2}\right) \lambda_{\max }(\log \varepsilon) 379(4.42) 252(3.96) \mathrm{nm} ;{ }^{1} \mathrm{H} \mathrm{NMR}\left(300 \mathrm{MHz}, \mathrm{CDCl}_{3}\right) \delta 6.98(\mathrm{~s}, 2 \mathrm{H})$, $6.96(\mathrm{~s}, 2 \mathrm{H}), 3.25(\mathrm{~s}, 2 \mathrm{H}), 3.18(\mathrm{~s}, 2 \mathrm{H}), 1.85-1.72(\mathrm{~m}, 8 \mathrm{H}), 1.45-1.37(\mathrm{~m}, 8 \mathrm{H}) ;{ }^{13} \mathrm{C}$ NMR $(75$ $\left.\mathrm{MHz}, \mathrm{CDCl}_{3}\right) \delta 144.8,139.8,136.2,132.4,123.0,120.7,31.4,31.3,27.4,26.9$; HRMS calcd for $\mathrm{C}_{24} \mathrm{H}_{24} \mathrm{~S}_{3}$ 408.1040, found 408.1032. Anal. Calcd for $\mathrm{C}_{24} \mathrm{H}_{24} \mathrm{~S}_{3}: \mathrm{C}, 70.54 ; \mathrm{H}, 5.92$. Found: $\mathrm{C}$, 70.27; H, 5.82 .

\section{Hexafluoroantimonate Salt of Radical Cation of Terthiophene $2\left(2^{\circ+} \mathrm{SbF}_{6}{ }^{-}\right)$.} Terthiophene $2(20.6 \mathrm{mg}, 0.050 \mathrm{mmol})$ and $\mathrm{NO}^{+} \mathrm{SbF}_{6}{ }^{-}(13.4 \mathrm{mg}, 0.050 \mathrm{mmol})$ were placed in a Pyrex tube connectable to a vacuum line under an argon atmosphere and the tube was evacuated. On the other hand, $2 \mathrm{~mL}$ of $\mathrm{CH}_{2} \mathrm{Cl}_{2}$ was dried over $\mathrm{CaH}_{2}$, degassed by three freeze-pump-thaw cycles, and vapor-transferred directly into the cooled tube containing 2 and $\mathrm{NO}^{+} \mathrm{SbF}_{6}{ }^{-}$. Then the connection to a vacuum line was closed, and the Pyrex tube was warmed to room temperature. When 2 and $\mathrm{NO}^{+} \mathrm{SbF}_{6}^{-}$were completely dissolved in dry $\mathrm{CH}_{2} \mathrm{Cl}_{2}$ by mixing, the color of the solution turned to dark blue. After 5-minute mixing, the tube was connected to the vacuum line again, and $10 \mathrm{~mL}$ of hexane, which had been dried and degassed, was vapor-transferred onto the blue solution at $-78^{\circ} \mathrm{C}$. Slow diffusion of hexane under an argon atmosphere at room temperature for two weeks gave $28.5 \mathrm{mg}$ of $2^{\cdot+} \mathrm{SbF}_{6}{ }^{-}$salts $(88 \%)$ as a dark blue solid: $\mathrm{mp}(\mathrm{dec})$ 219.4-220.2 ${ }^{\circ} \mathrm{C}$; UV-vis-NIR $\left(\mathrm{CH}_{2} \mathrm{Cl}_{2}\right) \quad \lambda_{\max }(\log \varepsilon) \quad 618(4.73) \quad 931(4.51) \mathrm{nm}$. Anal. Calcd for $\mathrm{C}_{24} \mathrm{H}_{24} \mathrm{~F}_{6} \mathrm{~S}_{3} \mathrm{Sb}: \mathrm{C}, 44.73 ; \mathrm{H}, 3.75$. Found: $\mathrm{C}, 44.33 ; \mathrm{H}, 3.76$.

\section{Reference}

1. Wakamiya, A.; Yamazaki, D.; Nishinaga, T.; Kitagawa, T.; Komatsu, K. J. Org. Chem. 2003, $68,8305-8314$. 


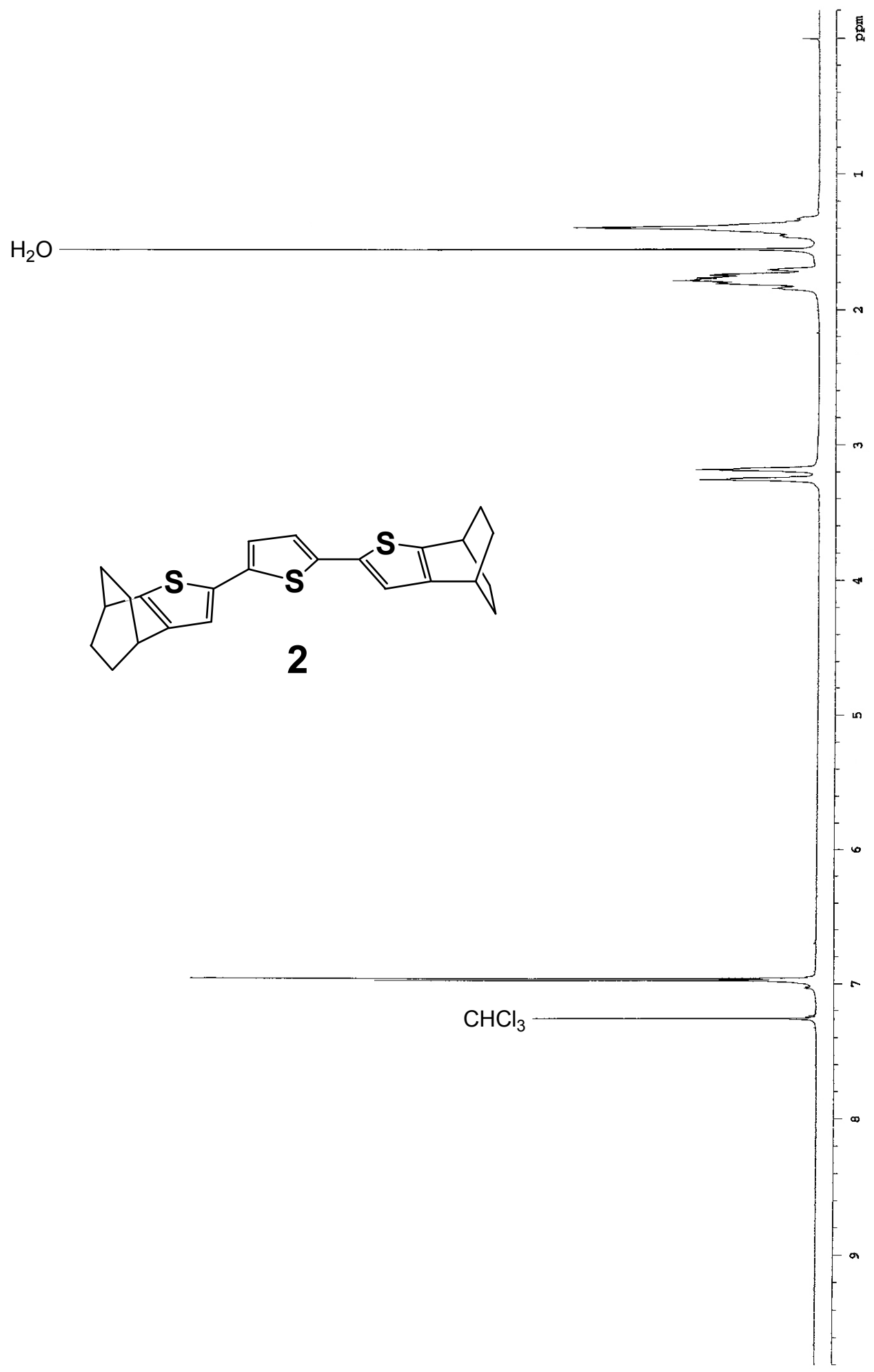

Figure S1. ${ }^{1} \mathrm{H}$ NMR (300 MHz) spectrum of 2 


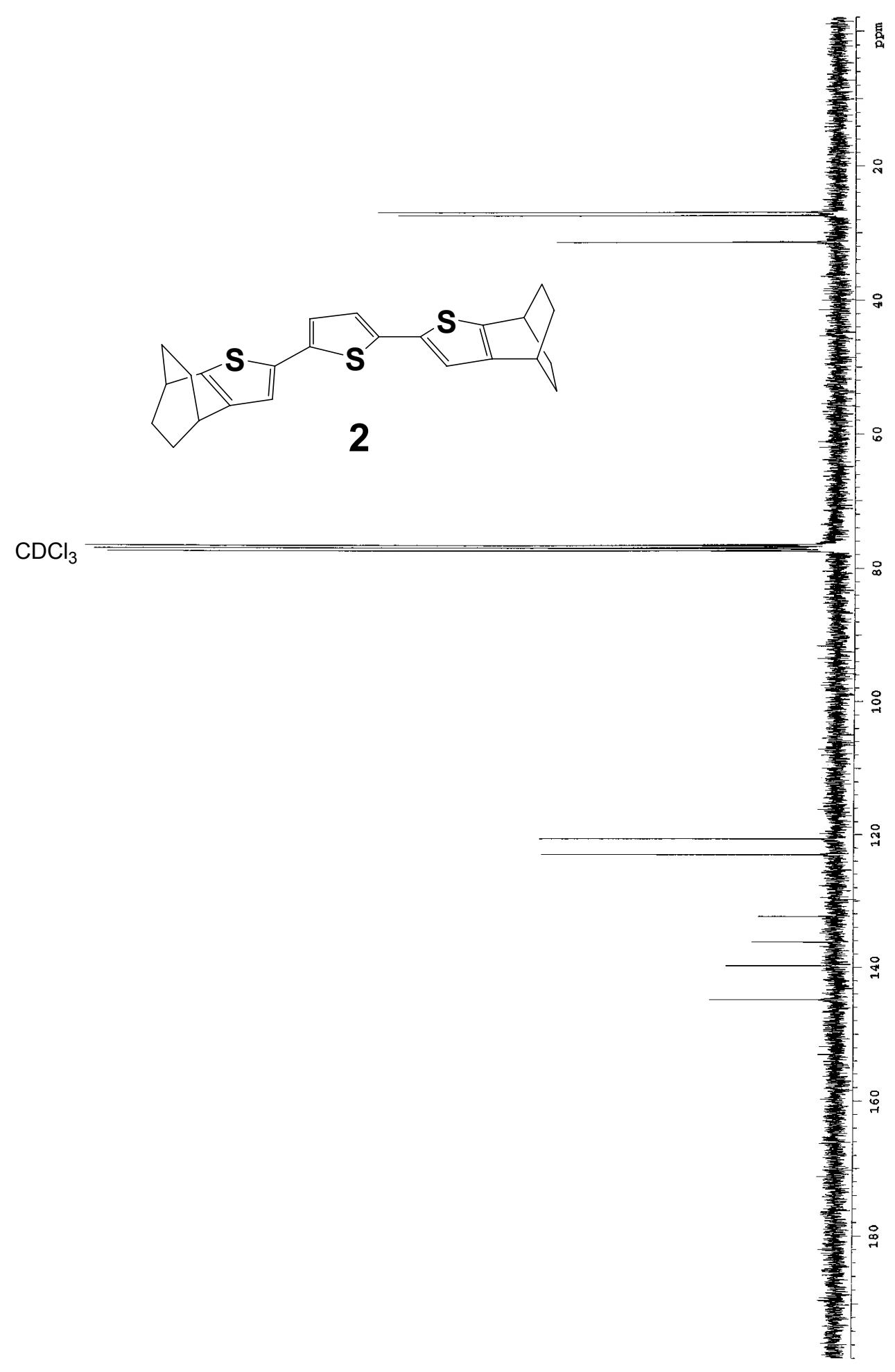

Figure S2. ${ }^{13} \mathrm{C}$ NMR (75.4 MHz) spectrum of 2 
X-Ray Structural Determination. The intensity data were collected on a Bruker SMART APEX equipped with a CCD area detector with graphite monochromated MoK $\alpha$ radiation. Frames corresponding to an arbitrary hemispher of data were collected at $-150{ }^{\circ} \mathrm{C}$ using $\omega$ scans of $0.3^{\circ}$ counted for a total of $10 \mathrm{~s}$ per frame. The structures were solved using Bruker SHELXTL program package. Crystallographic data for terthiophene 2, $2^{+} \mathrm{SbF}_{6}^{-} \cdot\left(\mathrm{CH}_{2} \mathrm{Cl}_{2}\right)_{2}$ are given in the CIF files.

\section{X-Ray Crystallography of 2.}
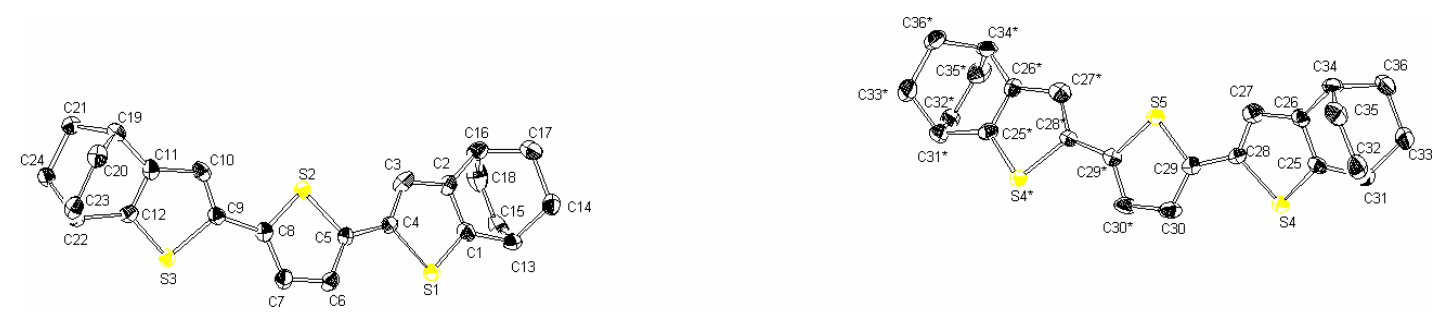
Table 1. Crystal data and structure refinement for $\mathbf{2}$.

Identification code

Empirical formula

Formula weight

Temperature

Wavelength

Crystal system

Space group

Unit cell dimensions

Volume

Z

Density (calculated)

Absorption coefficient

$\mathrm{F}(000)$

Crystal size

Theta range for data collection

Index ranges

Reflections collected

Independent reflections

Completeness to theta $=25.00^{\circ}$

Absorption correction

Max. and min. transmission

Refinement method

Data / restraints / parameters

Goodness-of-fit on $\mathrm{F}^{2}$

Final $\mathrm{R}$ indices $[\mathrm{I}>2 \operatorname{sigma}(\mathrm{I})]$

$\mathrm{R}$ indices (all data)

Largest diff. peak and hole
2

C24 H24 S3

408.61

100(2) K

$0.71073 \AA$

Monoclinic

$\mathrm{C} 2 / \mathrm{c}$

$$
\begin{array}{ll}
\mathrm{a}=40.384(4) \AA & \alpha=90^{\circ} . \\
\mathrm{b}=6.3078(6) \AA & \beta=124.144(2)^{\circ} . \\
\mathrm{c}=27.942(3) \AA & \gamma=90^{\circ} .
\end{array}
$$

5890.9(10) $\AA^{3}$

12

$1.382 \mathrm{Mg} / \mathrm{m}^{3}$

$0.384 \mathrm{~mm}^{-1}$

2592

$0.30 \times 0.30 \times 0.10 \mathrm{~mm}^{3}$

2.06 to $25.00^{\circ}$.

$-48<=\mathrm{h}<=47,-7<=\mathrm{k}<=7,-33<=1<=26$

14701

$5186[\mathrm{R}($ int $)=0.0370]$

$99.8 \%$

Empirical

0.9626 and 0.8934

Full-matrix least-squares on $\mathrm{F}^{2}$

$5186 / 2 / 423$

1.001

$\mathrm{R} 1=0.0375, \mathrm{wR} 2=0.0754$

$\mathrm{R} 1=0.0544, \mathrm{wR} 2=0.0792$

0.735 and -0.251 e. $\AA^{-3}$ 
Table 2. Atomic coordinates $\left(\times 10^{4}\right)$ and equivalent isotropic displacement parameters $\left(\AA^{2} \times 10^{3}\right)$ for 2. $U(e q)$ is defined as one third of the trace of the orthogonalized $U^{i j}$ tensor.

\begin{tabular}{|c|c|c|c|c|}
\hline & $\mathrm{x}$ & $\mathrm{y}$ & $\mathrm{z}$ & $\mathrm{U}(\mathrm{eq})$ \\
\hline $\mathrm{S}(2)$ & $7247(1)$ & $8767(1)$ & $1532(1)$ & $23(1)$ \\
\hline $\mathrm{S}(1)$ & $6655(1)$ & $11851(2)$ & $2259(1)$ & $27(1)$ \\
\hline $\mathrm{C}(3)$ & $6599(2)$ & $8157(9)$ & $1862(4)$ & $33(2)$ \\
\hline$C(1)$ & $6325(1)$ & $10079(4)$ & $2234(1)$ & $27(1)$ \\
\hline$C(2)$ & $6321(1)$ & $8166(3)$ & $2017(1)$ & $24(1)$ \\
\hline$C(103)$ & $6626(9)$ & $11120(30)$ & $2183(12)$ & $107(17)$ \\
\hline$S(101)$ & $6616(3)$ & $7489(13)$ & $1795(4)$ & $27(2)$ \\
\hline $\mathrm{S}(3)$ & $7812(1)$ & $12033(2)$ & $836(1)$ & $23(1)$ \\
\hline$C(10)$ & $7928(2)$ & $8234(8)$ & $1215(3)$ & $26(2)$ \\
\hline $\mathrm{C}(11)$ & $8107(1)$ & $8318(3)$ & $898(1)$ & $24(1)$ \\
\hline$C(12)$ & $8063(1)$ & $10287(4)$ & $678(1)$ & $24(1)$ \\
\hline $\mathrm{C}(110)$ & $7845(9)$ & $11240(30)$ & $885(12)$ & $125(18)$ \\
\hline$S(103)$ & $7959(3)$ & $7541(12)$ & $1324(4)$ & $23(2)$ \\
\hline$S(4)$ & $5318(1)$ & $6016(2)$ & $9095(1)$ & $23(1)$ \\
\hline$C(27)$ & $5460(2)$ & $2279(9)$ & $8899(3)$ & $29(2)$ \\
\hline $\mathrm{C}(127)$ & $5345(6)$ & $5160(40)$ & $9050(11)$ & $78(11)$ \\
\hline$S(104)$ & $5481(2)$ & $1587(11)$ & $8831(3)$ & $23(1)$ \\
\hline$S(5)$ & 5000 & $2869(1)$ & 7500 & $25(1)$ \\
\hline$C(7)$ & $7522(1)$ & $12531(3)$ & $1697(1)$ & $27(1)$ \\
\hline$C(4)$ & $6805(1)$ & $9989(3)$ & 1961(1) & $20(1)$ \\
\hline$C(6)$ & $7267(1)$ & $12503(3)$ & $1892(1)$ & $26(1)$ \\
\hline$C(19)$ & $8334(1)$ & $6840(3)$ & $758(1)$ & $26(1)$ \\
\hline$C(25)$ & $5536(1)$ & $4204(3)$ & $9636(1)$ & $25(1)$ \\
\hline$C(8)$ & $7545(1)$ & $10622(3)$ & $1489(1)$ & $22(1)$ \\
\hline$C(29)$ & $5143(1)$ & $4764(3)$ & $8030(1)$ & $22(1)$ \\
\hline$C(5)$ & $7095(1)$ & $10587(3)$ & $1835(1)$ & $20(1)$ \\
\hline$C(28)$ & $5302(1)$ & $4148(4)$ & $8619(1)$ & $20(1)$ \\
\hline$C(24)$ & $8066(1)$ & $9028(3)$ & $-145(1)$ & $25(1)$ \\
\hline$C(9)$ & $7761(1)$ & $10080(4)$ & 1231(1) & $22(1)$ \\
\hline$C(14)$ & $6122(1)$ & $8621(3)$ & $2838(1)$ & $31(1)$ \\
\hline$C(34)$ & $5816(1)$ & $779(3)$ & 9993(1) & $29(1)$ \\
\hline
\end{tabular}




\begin{tabular}{lrrrr}
$\mathrm{C}(22)$ & $8249(1)$ & $10651(3)$ & $352(1)$ & $25(1)$ \\
$\mathrm{C}(30)$ & $5081(1)$ & $6728(3)$ & $7797(1)$ & $32(1)$ \\
$\mathrm{C}(31)$ & $5686(1)$ & $4487(4)$ & $10259(1)$ & $34(1)$ \\
$\mathrm{C}(17)$ & $6096(1)$ & $6428(3)$ & $2576(1)$ & $32(1)$ \\
$\mathrm{C}(23)$ & $8696(1)$ & $10119(4)$ & $768(1)$ & $30(1)$ \\
$\mathrm{C}(35)$ & $6218(1)$ & $1854(4)$ & $10445(1)$ & $38(1)$ \\
$\mathrm{C}(16)$ & $6014(1)$ & $6681(4)$ & $1972(1)$ & $32(1)$ \\
$\mathrm{C}(26)$ & $5598(1)$ & $2277(3)$ & $9494(1)$ & $23(1)$ \\
$\mathrm{C}(20)$ & $8748(1)$ & $7842(4)$ & $1006(1)$ & $32(1)$ \\
$\mathrm{C}(15)$ & $5616(1)$ & $9940(4)$ & $1851(1)$ & $47(1)$ \\
$\mathrm{C}(21)$ & $8111(1)$ & $6763(3)$ & $98(1)$ & $27(1)$ \\
$\mathrm{C}(36)$ & $5575(1)$ & $556(4)$ & $10262(1)$ & $38(1)$ \\
$\mathrm{C}(13)$ & $6034(1)$ & $10370(4)$ & $2399(1)$ & $33(1)$ \\
$\mathrm{C}(18)$ & $5607(1)$ & $7777(4)$ & $1589(1)$ & $45(1)$ \\
$\mathrm{C}(32)$ & $6140(1)$ & $4017(4)$ & $10617(1)$ & $40(1)$ \\
$\mathrm{C}(33)$ & $5485(1)$ & $2766(4)$ & $10401(1)$ & $43(1)$ \\
& & & & \\
\hline
\end{tabular}


Table 3. Bond lengths $[\AA]$ and angles $\left[{ }^{\circ}\right]$ for $\mathbf{2}$.

\begin{tabular}{|c|c|}
\hline $\mathrm{S}(2)-\mathrm{C}(8)$ & $1.731(2)$ \\
\hline$S(2)-C(5)$ & $1.733(2)$ \\
\hline $\mathrm{S}(1)-\mathrm{C}(1)$ & $1.712(2)$ \\
\hline $\mathrm{S}(1)-\mathrm{C}(4)$ & $1.733(2)$ \\
\hline$C(3)-C(4)$ & $1.357(7)$ \\
\hline$C(3)-C(2)$ & $1.416(7)$ \\
\hline $\mathrm{C}(1)-\mathrm{C}(2)$ & $1.345(3)$ \\
\hline $\mathrm{C}(1)-\mathrm{C}(103)$ & $1.46(3)$ \\
\hline$C(1)-C(13)$ & $1.494(3)$ \\
\hline$C(2)-C(16)$ & $1.501(3)$ \\
\hline$C(2)-S(101)$ & $1.680(7)$ \\
\hline$C(103)-C(4)$ & $1.39(2)$ \\
\hline$S(101)-C(4)$ & $1.698(8)$ \\
\hline$S(3)-C(12)$ & $1.717(2)$ \\
\hline $\mathrm{S}(3)-\mathrm{C}(9)$ & $1.738(2)$ \\
\hline$C(10)-C(9)$ & $1.359(6)$ \\
\hline $\mathrm{C}(10)-\mathrm{C}(11)$ & $1.422(7)$ \\
\hline $\mathrm{C}(11)-\mathrm{C}(12)$ & $1.353(3)$ \\
\hline $\mathrm{C}(11)-\mathrm{C}(19)$ & $1.505(3)$ \\
\hline$C(11)-S(103)$ & $1.678(7)$ \\
\hline$C(12)-C(110)$ & $1.43(3)$ \\
\hline $\mathrm{C}(12)-\mathrm{C}(22)$ & $1.488(3)$ \\
\hline $\mathrm{C}(110)-\mathrm{C}(9)$ & $1.39(2)$ \\
\hline $\mathrm{S}(103)-\mathrm{C}(9)$ & $1.743(7)$ \\
\hline$S(4)-C(25)$ & $1.695(2)$ \\
\hline $\mathrm{S}(4)-\mathrm{C}(28)$ & $1.750(2)$ \\
\hline $\mathrm{C}(27)-\mathrm{C}(28)$ & $1.359(7)$ \\
\hline$C(27)-C(26)$ & $1.427(8)$ \\
\hline $\mathrm{C}(127)-\mathrm{C}(28)$ & $1.28(3)$ \\
\hline$C(127)-C(25)$ & $1.49(2)$ \\
\hline$S(104)-C(26)$ & $1.692(7)$ \\
\hline$S(104)-C(28)$ & $1.732(6)$ \\
\hline$S(5)-C(29)$ & $1.730(2)$ \\
\hline$S(5)-C(29) \# 1$ & $1.730(2)$ \\
\hline
\end{tabular}




\begin{tabular}{|c|c|}
\hline$C(7)-C(8)$ & $1.363(3)$ \\
\hline$C(7)-C(6)$ & $1.410(3)$ \\
\hline C(4)-C(5) & $1.450(3)$ \\
\hline$C(6)-C(5)$ & $1.359(3)$ \\
\hline$C(19)-C(21)$ & $1.535(3)$ \\
\hline C(19)-C(20) & $1.540(3)$ \\
\hline$C(25)-C(26)$ & $1.346(3)$ \\
\hline $\mathrm{C}(25)-\mathrm{C}(31)$ & $1.497(3)$ \\
\hline $\mathrm{C}(8)-\mathrm{C}(9)$ & $1.451(3)$ \\
\hline C(29)-C(30) & $1.355(3)$ \\
\hline C(29)-C(28) & $1.446(3)$ \\
\hline $\mathrm{C}(24)-\mathrm{C}(22)$ & $1.539(3)$ \\
\hline $\mathrm{C}(24)-\mathrm{C}(21)$ & $1.548(3)$ \\
\hline$C(14)-C(13)$ & $1.537(3)$ \\
\hline C(14)-C(17) & $1.541(3)$ \\
\hline$C(34)-C(26)$ & $1.494(3)$ \\
\hline$C(34)-C(36)$ & $1.536(3)$ \\
\hline$C(34)-C(35)$ & $1.544(3)$ \\
\hline$C(22)-C(23)$ & $1.540(3)$ \\
\hline$C(30)-C(30) \# 1$ & $1.404(4)$ \\
\hline$C(31)-C(33)$ & $1.534(3)$ \\
\hline$C(31)-C(32)$ & $1.547(3)$ \\
\hline C(17)-C(16) & $1.533(3)$ \\
\hline$C(23)-C(20)$ & $1.546(3)$ \\
\hline $\mathrm{C}(35)-\mathrm{C}(32)$ & $1.536(3)$ \\
\hline C(16)-C(18) & $1.534(3)$ \\
\hline$C(15)-C(18)$ & $1.538(3)$ \\
\hline$C(15)-C(13)$ & $1.539(3)$ \\
\hline$C(36)-C(33)$ & $1.543(3)$ \\
\hline $\mathrm{C}(8)-\mathrm{S}(2)-\mathrm{C}(5)$ & $92.18(10)$ \\
\hline$C(1)-S(1)-C(4)$ & $90.62(12)$ \\
\hline $\mathrm{C}(4)-\mathrm{C}(3)-\mathrm{C}(2)$ & $115.7(4)$ \\
\hline$C(2)-C(1)-C(103)$ & $99.3(6)$ \\
\hline$C(2)-C(1)-C(13)$ & $115.8(2)$ \\
\hline$C(103)-C(1)-C(13)$ & $144.9(6)$ \\
\hline
\end{tabular}




\begin{tabular}{|c|c|}
\hline $\mathrm{C}(2)-\mathrm{C}(1)-\mathrm{S}(1)$ & $114.62(17)$ \\
\hline$C(103)-C(1)-S(1)$ & $15.4(6)$ \\
\hline $\mathrm{C}(13)-\mathrm{C}(1)-\mathrm{S}(1)$ & $129.53(18)$ \\
\hline $\mathrm{C}(1)-\mathrm{C}(2)-\mathrm{C}(3)$ & 109.3(3) \\
\hline$C(1)-C(2)-C(16)$ & $113.46(19)$ \\
\hline$C(3)-C(2)-C(16)$ & 137.1(3) \\
\hline $\mathrm{C}(1)-\mathrm{C}(2)-\mathrm{S}(101)$ & $124.2(3)$ \\
\hline$C(3)-C(2)-S(101)$ & $14.9(4)$ \\
\hline$C(16)-C(2)-S(101)$ & $122.3(3)$ \\
\hline$C(4)-C(103)-C(1)$ & $119.0(11)$ \\
\hline$C(2)-S(101)-C(4)$ & $88.0(4)$ \\
\hline $\mathrm{C}(12)-\mathrm{S}(3)-\mathrm{C}(9)$ & $90.32(12)$ \\
\hline $\mathrm{C}(9)-\mathrm{C}(10)-\mathrm{C}(11)$ & $115.0(3)$ \\
\hline$C(12)-C(11)-C(10)$ & $109.6(3)$ \\
\hline $\mathrm{C}(12)-\mathrm{C}(11)-\mathrm{C}(19)$ & $113.17(19)$ \\
\hline $\mathrm{C}(10)-\mathrm{C}(11)-\mathrm{C}(19)$ & $137.3(3)$ \\
\hline C(12)-C(11)-S(103) & $125.7(3)$ \\
\hline C(10)-C(11)-S(103) & $16.3(4)$ \\
\hline C(19)-C(11)-S(103) & $121.0(3)$ \\
\hline $\mathrm{C}(11)-\mathrm{C}(12)-\mathrm{C}(110)$ & $98.8(6)$ \\
\hline $\mathrm{C}(11)-\mathrm{C}(12)-\mathrm{C}(22)$ & $116.0(2)$ \\
\hline $\mathrm{C}(110)-\mathrm{C}(12)-\mathrm{C}(22)$ & $145.2(6)$ \\
\hline $\mathrm{C}(11)-\mathrm{C}(12)-\mathrm{S}(3)$ & $114.61(18)$ \\
\hline $\mathrm{C}(110)-\mathrm{C}(12)-\mathrm{S}(3)$ & $15.8(6)$ \\
\hline$C(22)-C(12)-S(3)$ & $129.37(17)$ \\
\hline $\mathrm{C}(9)-\mathrm{C}(110)-\mathrm{C}(12)$ & $120.4(12)$ \\
\hline $\mathrm{C}(11)-\mathrm{S}(103)-\mathrm{C}(9)$ & $86.6(3)$ \\
\hline $\mathrm{C}(25)-\mathrm{S}(4)-\mathrm{C}(28)$ & $90.21(12)$ \\
\hline$C(28)-C(27)-C(26)$ & $114.9(4)$ \\
\hline $\mathrm{C}(28)-\mathrm{C}(127)-\mathrm{C}(25)$ & $123.1(16)$ \\
\hline $\mathrm{C}(26)-\mathrm{S}(104)-\mathrm{C}(28)$ & $86.6(3)$ \\
\hline $\mathrm{C}(29)-\mathrm{S}(5)-\mathrm{C}(29) \# 1$ & $92.60(15)$ \\
\hline$C(8)-C(7)-C(6)$ & $113.2(2)$ \\
\hline$C(3)-C(4)-C(103)$ & $96.8(10)$ \\
\hline$C(3)-C(4)-C(5)$ & $131.0(3)$ \\
\hline$C(103)-C(4)-C(5)$ & 132.1(10) \\
\hline
\end{tabular}




\begin{tabular}{|c|c|}
\hline$C(3)-C(4)-S(101)$ & $12.8(4)$ \\
\hline$C(103)-C(4)-S(101)$ & $109.5(10)$ \\
\hline$C(5)-C(4)-S(101)$ & $118.3(3)$ \\
\hline$C(3)-C(4)-S(1)$ & $109.8(3)$ \\
\hline$C(103)-C(4)-S(1)$ & $13.0(10)$ \\
\hline$C(5)-C(4)-S(1)$ & $119.15(16)$ \\
\hline$S(101)-C(4)-S(1)$ & $122.5(3)$ \\
\hline$C(5)-C(6)-C(7)$ & $113.8(2)$ \\
\hline$C(11)-C(19)-C(21)$ & $107.50(17)$ \\
\hline $\mathrm{C}(11)-\mathrm{C}(19)-\mathrm{C}(20)$ & $107.13(17)$ \\
\hline $\mathrm{C}(21)-\mathrm{C}(19)-\mathrm{C}(20)$ & $108.01(18)$ \\
\hline $\mathrm{C}(26)-\mathrm{C}(25)-\mathrm{C}(127)$ & $96.1(11)$ \\
\hline $\mathrm{C}(26)-\mathrm{C}(25)-\mathrm{C}(31)$ & $115.5(2)$ \\
\hline $\mathrm{C}(127)-\mathrm{C}(25)-\mathrm{C}(31)$ & $148.4(11)$ \\
\hline$C(26)-C(25)-S(4)$ & $115.74(18)$ \\
\hline$C(127)-C(25)-S(4)$ & $19.7(10)$ \\
\hline $\mathrm{C}(31)-\mathrm{C}(25)-\mathrm{S}(4)$ & $128.71(18)$ \\
\hline $\mathrm{C}(7)-\mathrm{C}(8)-\mathrm{C}(9)$ & $128.7(2)$ \\
\hline $\mathrm{C}(7)-\mathrm{C}(8)-\mathrm{S}(2)$ & $110.52(16)$ \\
\hline $\mathrm{C}(9)-\mathrm{C}(8)-\mathrm{S}(2)$ & $120.71(16)$ \\
\hline $\mathrm{C}(30)-\mathrm{C}(29)-\mathrm{C}(28)$ & $129.5(2)$ \\
\hline $\mathrm{C}(30)-\mathrm{C}(29)-\mathrm{S}(5)$ & $109.81(16)$ \\
\hline $\mathrm{C}(28)-\mathrm{C}(29)-\mathrm{S}(5)$ & $120.72(17)$ \\
\hline$C(6)-C(5)-C(4)$ & $129.3(2)$ \\
\hline$C(6)-C(5)-S(2)$ & $110.31(16)$ \\
\hline$C(4)-C(5)-S(2)$ & $120.29(16)$ \\
\hline $\mathrm{C}(127)-\mathrm{C}(28)-\mathrm{C}(27)$ & $96.7(9)$ \\
\hline $\mathrm{C}(127)-\mathrm{C}(28)-\mathrm{C}(29)$ & $132.5(9)$ \\
\hline $\mathrm{C}(27)-\mathrm{C}(28)-\mathrm{C}(29)$ & $130.8(3)$ \\
\hline $\mathrm{C}(127)-\mathrm{C}(28)-\mathrm{S}(104)$ & 109.3(9) \\
\hline $\mathrm{C}(27)-\mathrm{C}(28)-\mathrm{S}(104)$ & $12.5(4)$ \\
\hline C(29)-C(28)-S(104) & $118.2(3)$ \\
\hline $\mathrm{C}(127)-\mathrm{C}(28)-\mathrm{S}(4)$ & $13.3(9)$ \\
\hline$C(27)-C(28)-S(4)$ & $110.0(3)$ \\
\hline $\mathrm{C}(29)-\mathrm{C}(28)-\mathrm{S}(4)$ & $119.22(17)$ \\
\hline$S(104)-C(28)-S(4)$ & $122.5(2)$ \\
\hline
\end{tabular}




\begin{tabular}{|c|c|}
\hline$C(22)-C(24)-C(21)$ & $109.87(17)$ \\
\hline $\mathrm{C}(10)-\mathrm{C}(9)-\mathrm{C}(110)$ & $96.2(11)$ \\
\hline C(10)-C(9)-C(8) & $131.8(3)$ \\
\hline$C(110)-C(9)-C(8)$ & $131.9(11)$ \\
\hline$C(10)-C(9)-S(3)$ & $110.5(3)$ \\
\hline$C(110)-C(9)-S(3)$ & $14.3(11)$ \\
\hline $\mathrm{C}(8)-\mathrm{C}(9)-\mathrm{S}(3)$ & $117.67(17)$ \\
\hline C(10)-C(9)-S(103) & $12.4(4)$ \\
\hline C(110)-C(9)-S(103) & $108.4(11)$ \\
\hline$C(8)-C(9)-S(103)$ & $119.6(3)$ \\
\hline$S(3)-C(9)-S(103)$ & $122.7(3)$ \\
\hline$C(13)-C(14)-C(17)$ & 109.91(18) \\
\hline$C(26)-C(34)-C(36)$ & $108.13(18)$ \\
\hline$C(26)-C(34)-C(35)$ & $106.50(18)$ \\
\hline $\mathrm{C}(36)-\mathrm{C}(34)-\mathrm{C}(35)$ & $107.65(19)$ \\
\hline $\mathrm{C}(12)-\mathrm{C}(22)-\mathrm{C}(24)$ & $107.26(17)$ \\
\hline$C(12)-C(22)-C(23)$ & $106.50(17)$ \\
\hline$C(24)-C(22)-C(23)$ & $107.69(17)$ \\
\hline $\mathrm{C}(29)-\mathrm{C}(30)-\mathrm{C}(30) \# 1$ & $113.89(13)$ \\
\hline$C(25)-C(31)-C(33)$ & $106.74(18)$ \\
\hline $\mathrm{C}(25)-\mathrm{C}(31)-\mathrm{C}(32)$ & $106.78(18)$ \\
\hline $\mathrm{C}(33)-\mathrm{C}(31)-\mathrm{C}(32)$ & $107.7(2)$ \\
\hline$C(16)-C(17)-C(14)$ & $110.17(18)$ \\
\hline$C(22)-C(23)-C(20)$ & $110.19(17)$ \\
\hline$C(32)-C(35)-C(34)$ & 109.94(19) \\
\hline C(2)-C(16)-C(17) & $108.29(18)$ \\
\hline$C(2)-C(16)-C(18)$ & $106.74(19)$ \\
\hline$C(17)-C(16)-C(18)$ & $107.53(19)$ \\
\hline$C(25)-C(26)-C(27)$ & 109.1(3) \\
\hline$C(25)-C(26)-C(34)$ & $113.9(2)$ \\
\hline$C(27)-C(26)-C(34)$ & $136.7(3)$ \\
\hline$C(25)-C(26)-S(104)$ & $124.9(3)$ \\
\hline C(27)-C(26)-S(104) & $15.8(4)$ \\
\hline$C(34)-C(26)-S(104)$ & $121.0(3)$ \\
\hline $\mathrm{C}(19)-\mathrm{C}(20)-\mathrm{C}(23)$ & $109.74(17)$ \\
\hline$C(18)-C(15)-C(13)$ & $110.47(18)$ \\
\hline
\end{tabular}




$\begin{array}{ll}\mathrm{C}(19)-\mathrm{C}(21)-\mathrm{C}(24) & 110.08(17) \\ \mathrm{C}(34)-\mathrm{C}(36)-\mathrm{C}(33) & 110.04(18) \\ \mathrm{C}(1)-\mathrm{C}(13)-\mathrm{C}(14) & 106.78(18) \\ \mathrm{C}(1)-\mathrm{C}(13)-\mathrm{C}(15) & 106.58(19) \\ \mathrm{C}(14)-\mathrm{C}(13)-\mathrm{C}(15) & 107.53(18) \\ \mathrm{C}(16)-\mathrm{C}(18)-\mathrm{C}(15) & 109.7(2) \\ \mathrm{C}(35)-\mathrm{C}(32)-\mathrm{C}(31) & 110.10(19) \\ \mathrm{C}(31)-\mathrm{C}(33)-\mathrm{C}(36) & 110.01(19)\end{array}$

Symmetry transformations used to generate equivalent atoms: $\# 1-\mathrm{x}+1, \mathrm{y},-\mathrm{z}+3 / 2$ 
Table 4. Anisotropic displacement parameters $\left(\AA^{2} \times 10^{3}\right)$ for 2 . The anisotropic displacement factor exponent takes the form: $\quad-2 \pi^{2}\left[\mathrm{~h}^{2} \mathrm{a}^{* 2} \mathrm{U}^{11}+\ldots \quad+2 \mathrm{hk} \mathrm{a} \mathrm{b}^{*} \mathrm{U}^{12}\right]$

\begin{tabular}{|c|c|c|c|c|c|c|}
\hline & $\mathrm{U}^{11}$ & $\mathrm{U}^{22}$ & $\mathrm{U}^{33}$ & $\mathrm{U}^{23}$ & $\mathrm{U}^{13}$ & $\mathrm{U}^{12}$ \\
\hline$S(2)$ & $23(1)$ & $24(1)$ & $28(1)$ & $-2(1)$ & $17(1)$ & $-2(1)$ \\
\hline $\mathrm{S}(1)$ & $35(1)$ & 21(1) & $40(1)$ & $-2(1)$ & $30(1)$ & $-3(1)$ \\
\hline$C(3)$ & $33(3)$ & $36(4)$ & $31(3)$ & $-11(3)$ & $18(2)$ & $0(3)$ \\
\hline $\mathrm{C}(1)$ & $25(1)$ & $26(1)$ & $30(1)$ & 2(1) & $17(1)$ & $-2(1)$ \\
\hline $\mathrm{C}(2)$ & $18(1)$ & $31(1)$ & $25(1)$ & $-3(1)$ & $13(1)$ & $-1(1)$ \\
\hline$C(103)$ & $130(20)$ & $60(20)$ & $68(17)$ & $-32(15)$ & $18(15)$ & $-46(17)$ \\
\hline$S(101)$ & $25(2)$ & $31(4)$ & $38(3)$ & $-12(3)$ & $25(2)$ & $-14(2)$ \\
\hline $\mathrm{S}(3)$ & $25(1)$ & $25(1)$ & $26(1)$ & $-3(1)$ & $19(1)$ & $-4(1)$ \\
\hline $\mathrm{C}(10)$ & $24(2)$ & $27(4)$ & $23(3)$ & $2(2)$ & $11(2)$ & $-6(2)$ \\
\hline $\mathrm{C}(11)$ & 18(1) & $31(1)$ & $21(1)$ & $-3(1)$ & $10(1)$ & $-7(1)$ \\
\hline$C(12)$ & $20(1)$ & $24(1)$ & $28(1)$ & $-5(1)$ & $14(1)$ & $-3(1)$ \\
\hline$C(110)$ & $100(20)$ & $60(20)$ & $120(30)$ & $-39(18)$ & $4(19)$ & $-22(16)$ \\
\hline$S(103)$ & $25(2)$ & $27(4)$ & $27(3)$ & $-5(3)$ & $20(2)$ & $-5(2)$ \\
\hline $\mathrm{S}(4)$ & $27(1)$ & $20(1)$ & $23(1)$ & $1(1)$ & $14(1)$ & $1(1)$ \\
\hline$C(27)$ & $22(2)$ & $28(4)$ & $34(3)$ & $-8(3)$ & $13(2)$ & $-9(3)$ \\
\hline $\mathrm{C}(127)$ & $47(10)$ & $29(13)$ & $160(30)$ & $27(13)$ & $59(14)$ & $20(10)$ \\
\hline$S(104)$ & $19(2)$ & $25(3)$ & $16(2)$ & $6(2)$ & $5(2)$ & $-3(2)$ \\
\hline $\mathrm{S}(5)$ & $32(1)$ & $18(1)$ & $24(1)$ & 0 & $15(1)$ & 0 \\
\hline$C(7)$ & $27(1)$ & $30(1)$ & $26(1)$ & $-8(1)$ & $16(1)$ & $-10(1)$ \\
\hline $\mathrm{C}(4)$ & $17(1)$ & $21(1)$ & $17(1)$ & $-3(1)$ & $8(1)$ & $0(1)$ \\
\hline $\mathrm{C}(6)$ & $25(1)$ & $28(1)$ & $25(1)$ & $-7(1)$ & $14(1)$ & $-4(1)$ \\
\hline C(19) & $28(1)$ & $22(1)$ & $27(1)$ & $4(1)$ & $15(1)$ & $0(1)$ \\
\hline$C(25)$ & $23(1)$ & $22(1)$ & $32(1)$ & $2(1)$ & $16(1)$ & $-3(1)$ \\
\hline $\mathrm{C}(8)$ & $16(1)$ & $28(1)$ & 21(1) & $0(1)$ & $9(1)$ & $-3(1)$ \\
\hline$C(29)$ & $15(1)$ & $23(1)$ & $24(1)$ & $-2(1)$ & $9(1)$ & $-1(1)$ \\
\hline C(5) & $16(1)$ & $26(1)$ & $15(1)$ & $-1(1)$ & $7(1)$ & $0(1)$ \\
\hline$C(28)$ & $16(1)$ & $19(1)$ & $24(1)$ & $-3(1)$ & $11(1)$ & $-1(1)$ \\
\hline$C(24)$ & $26(1)$ & $27(1)$ & $23(1)$ & $1(1)$ & $14(1)$ & $-2(1)$ \\
\hline $\mathrm{C}(9)$ & $20(1)$ & $24(1)$ & $23(1)$ & $-2(1)$ & $12(1)$ & $-6(1)$ \\
\hline$C(14)$ & $35(1)$ & $35(2)$ & $32(1)$ & $-1(1)$ & $23(1)$ & $-2(1)$ \\
\hline$C(34)$ & $35(1)$ & $18(1)$ & $31(1)$ & $-1(1)$ & $18(1)$ & $-2(1)$ \\
\hline
\end{tabular}




\begin{tabular}{lllllll}
$\mathrm{C}(22)$ & $28(1)$ & $20(1)$ & $31(1)$ & $0(1)$ & $20(1)$ & $-2(1)$ \\
$\mathrm{C}(30)$ & $39(1)$ & $20(1)$ & $24(1)$ & $-2(1)$ & $9(1)$ & $-1(1)$ \\
$\mathrm{C}(31)$ & $47(2)$ & $25(1)$ & $39(2)$ & $-8(1)$ & $31(1)$ & $-8(1)$ \\
$\mathrm{C}(17)$ & $29(1)$ & $25(1)$ & $42(2)$ & $6(1)$ & $21(1)$ & $1(1)$ \\
$\mathrm{C}(23)$ & $24(1)$ & $39(2)$ & $34(1)$ & $-12(1)$ & $20(1)$ & $-12(1)$ \\
$\mathrm{C}(35)$ & $35(1)$ & $41(2)$ & $25(1)$ & $9(1)$ & $8(1)$ & $-1(1)$ \\
$\mathrm{C}(16)$ & $29(1)$ & $29(1)$ & $42(2)$ & $-13(1)$ & $23(1)$ & $-9(1)$ \\
$\mathrm{C}(26)$ & $20(1)$ & $24(1)$ & $24(1)$ & $-2(1)$ & $12(1)$ & $-7(1)$ \\
$\mathrm{C}(20)$ & $25(1)$ & $41(2)$ & $28(1)$ & $2(1)$ & $14(1)$ & $4(1)$ \\
$\mathrm{C}(15)$ & $31(1)$ & $63(2)$ & $58(2)$ & $37(2)$ & $33(2)$ & $23(1)$ \\
$\mathrm{C}(21)$ & $31(1)$ & $23(1)$ & $29(1)$ & $-3(1)$ & $18(1)$ & $-2(1)$ \\
$\mathrm{C}(36)$ & $53(2)$ & $29(1)$ & $38(2)$ & $3(1)$ & $28(1)$ & $-8(1)$ \\
$\mathrm{C}(13)$ & $46(2)$ & $19(1)$ & $51(2)$ & $3(1)$ & $37(2)$ & $5(1)$ \\
$\mathrm{C}(18)$ & $23(1)$ & $76(2)$ & $34(2)$ & $1(1)$ & $15(1)$ & $-13(1)$ \\
$\mathrm{C}(32)$ & $48(2)$ & $46(2)$ & $24(1)$ & $-8(1)$ & $19(1)$ & $-19(1)$ \\
$\mathrm{C}(33)$ & $63(2)$ & $38(2)$ & $47(2)$ & $-7(1)$ & $42(2)$ & $-14(1)$ \\
\hline
\end{tabular}


Table 5. Hydrogen coordinates ( $\left.\times 10^{4}\right)$ and isotropic displacement parameters $\left(\AA^{2} \times 10^{3}\right)$ for 2.

\begin{tabular}{|c|c|c|c|c|}
\hline & $\mathrm{x}$ & $\mathrm{y}$ & $\mathrm{z}$ & $\mathrm{U}(\mathrm{eq})$ \\
\hline $\mathrm{H}(3)$ & 6640 & 6946 & 1698 & 40 \\
\hline $\mathrm{H}(103)$ & 6699 & 12552 & 2301 & 128 \\
\hline $\mathrm{H}(10)$ & 7926 & 6981 & 1401 & 32 \\
\hline $\mathrm{H}(110)$ & 7757 & 12662 & 786 & 150 \\
\hline $\mathrm{H}(27)$ & 5477 & 1066 & 8712 & 35 \\
\hline $\mathrm{H}(127)$ & 5252 & 6575 & 8993 & 94 \\
\hline $\mathrm{H}(7)$ & 7663 & 13757 & 1709 & 32 \\
\hline $\mathrm{H}(6)$ & 7220 & 13711 & 2049 & 31 \\
\hline $\mathrm{H}(19)$ & 8359 & 5395 & 923 & 31 \\
\hline $\mathrm{H}(24 \mathrm{~A})$ & 8202 & 9120 & -348 & 30 \\
\hline $\mathrm{H}(24 \mathrm{~B})$ & 7779 & 9351 & -427 & 30 \\
\hline $\mathrm{H}(14 \mathrm{~A})$ & 5926 & 8702 & 2944 & 38 \\
\hline $\mathrm{H}(14 \mathrm{~B})$ & 6393 & 8827 & 3193 & 38 \\
\hline $\mathrm{H}(34)$ & 5859 & -626 & 9870 & 35 \\
\hline $\mathrm{H}(22)$ & 8208 & 12139 & 205 & 30 \\
\hline $\mathrm{H}(30)$ & 5140 & 7991 & 8017 & 39 \\
\hline $\mathrm{H}(31)$ & 5628 & 5941 & 10337 & 40 \\
\hline $\mathrm{H}(17 \mathrm{~A})$ & 6351 & 5655 & 2830 & 38 \\
\hline $\mathrm{H}(17 \mathrm{~B})$ & 5879 & 5590 & 2549 & 38 \\
\hline$H(23 A)$ & 8824 & 11141 & 1093 & 36 \\
\hline $\mathrm{H}(23 \mathrm{~B})$ & 8831 & 10240 & 562 & 36 \\
\hline $\mathrm{H}(35 \mathrm{~A})$ & 6371 & 939 & 10791 & 46 \\
\hline $\mathrm{H}(35 \mathrm{~B})$ & 6378 & 2049 & 10281 & 46 \\
\hline $\mathrm{H}(16)$ & 6018 & 5281 & 1808 & 38 \\
\hline $\mathrm{H}(20 \mathrm{~A})$ & 8899 & 6974 & 896 & 38 \\
\hline $\mathrm{H}(20 \mathrm{~B})$ & 8901 & 7881 & 1433 & 38 \\
\hline $\mathrm{H}(15 \mathrm{~A})$ & 5415 & 9955 & 1948 & 56 \\
\hline $\mathrm{H}(15 \mathrm{~B})$ & 5546 & 11073 & 1563 & 56 \\
\hline $\mathrm{H}(21 \mathrm{~A})$ & 7844 & 6123 & -73 & 32 \\
\hline $\mathrm{H}(21 \mathrm{~B})$ & 8262 & 5869 & -9 & 32 \\
\hline $\mathrm{H}(36 \mathrm{~A})$ & 5729 & -292 & 10622 & 46 \\
\hline
\end{tabular}




\begin{tabular}{lrrrr}
$\mathrm{H}(36 \mathrm{~B})$ & 5321 & -194 & 9990 & 46 \\
$\mathrm{H}(13)$ & 6053 & 11817 & 2559 & 40 \\
$\mathrm{H}(18 \mathrm{~A})$ & 5395 & 6878 & 1559 & 54 \\
$\mathrm{H}(18 \mathrm{~B})$ & 5545 & 7980 & 1195 & 54 \\
$\mathrm{H}(32 \mathrm{~A})$ & 6280 & 5140 & 10549 & 48 \\
$\mathrm{H}(32 \mathrm{~B})$ & 6246 & 4012 & 11034 & 48 \\
$\mathrm{H}(33 \mathrm{~A})$ & 5192 & 3003 & 10171 & 52 \\
$\mathrm{H}(33 \mathrm{~B})$ & 5587 & 2843 & 10815 & 52 \\
& & & & \\
\hline
\end{tabular}


Table 6. Torsion angles $\left[{ }^{\circ}\right]$ for 2.

\begin{tabular}{lc}
\hline $\mathrm{C}(4)-\mathrm{S}(1)-\mathrm{C}(1)-\mathrm{C}(2)$ & $0.26(19)$ \\
$\mathrm{C}(4)-\mathrm{S}(1)-\mathrm{C}(1)-\mathrm{C}(103)$ & $-2(5)$ \\
$\mathrm{C}(4)-\mathrm{S}(1)-\mathrm{C}(1)-\mathrm{C}(13)$ & $-177.6(2)$ \\
$\mathrm{C}(103)-\mathrm{C}(1)-\mathrm{C}(2)-\mathrm{C}(3)$ & $0.2(13)$ \\
$\mathrm{C}(13)-\mathrm{C}(1)-\mathrm{C}(2)-\mathrm{C}(3)$ & $177.8(4)$ \\
$\mathrm{S}(1)-\mathrm{C}(1)-\mathrm{C}(2)-\mathrm{C}(3)$ & $-0.4(4)$ \\
$\mathrm{C}(103)-\mathrm{C}(1)-\mathrm{C}(2)-\mathrm{C}(16)$ & $-176.6(12)$ \\
$\mathrm{C}(13)-\mathrm{C}(1)-\mathrm{C}(2)-\mathrm{C}(16)$ & $1.0(3)$ \\
$\mathrm{S}(1)-\mathrm{C}(1)-\mathrm{C}(2)-\mathrm{C}(16)$ & $-177.10(16)$ \\
$\mathrm{C}(103)-\mathrm{C}(1)-\mathrm{C}(2)-\mathrm{S}(101)$ & $1.6(13)$ \\
$\mathrm{C}(13)-\mathrm{C}(1)-\mathrm{C}(2)-\mathrm{S}(101)$ & $179.2(5)$ \\
$\mathrm{S}(1)-\mathrm{C}(1)-\mathrm{C}(2)-\mathrm{S}(101)$ & $1.1(5)$ \\
$\mathrm{C}(4)-\mathrm{C}(3)-\mathrm{C}(2)-\mathrm{C}(1)$ & $0.3(7)$ \\
$\mathrm{C}(4)-\mathrm{C}(3)-\mathrm{C}(2)-\mathrm{C}(16)$ & $175.9(3)$ \\
$\mathrm{C}(4)-\mathrm{C}(3)-\mathrm{C}(2)-\mathrm{S}(101)$ & $-175(3)$ \\
$\mathrm{C}(2)-\mathrm{C}(1)-\mathrm{C}(103)-\mathrm{C}(4)$ & $-1(2)$ \\
$\mathrm{C}(13)-\mathrm{C}(1)-\mathrm{C}(103)-\mathrm{C}(4)$ & $-176.9(5)$ \\
$\mathrm{S}(1)-\mathrm{C}(1)-\mathrm{C}(103)-\mathrm{C}(4)$ & $177(7)$ \\
$\mathrm{C}(1)-\mathrm{C}(2)-\mathrm{S}(101)-\mathrm{C}(4)$ & $-1.7(6)$ \\
$\mathrm{C}(3)-\mathrm{C}(2)-\mathrm{S}(101)-\mathrm{C}(4)$ & $4(2)$ \\
$\mathrm{C}(16)-\mathrm{C}(2)-\mathrm{S}(101)-\mathrm{C}(4)$ & $-3.1(5)$ \\
$\mathrm{C}(9)-\mathrm{C}(10)-\mathrm{C}(11)-\mathrm{C}(12)$ & $176.3(2)$ \\
$\mathrm{C}(9)-\mathrm{C}(10)-\mathrm{C}(11)-\mathrm{C}(19)$ & $-0.5(6)$ \\
$\mathrm{C}(9)-\mathrm{C}(10)-\mathrm{C}(11)-\mathrm{S}(103)$ & $177.9(3)$ \\
$\mathrm{C}(10)-\mathrm{C}(11)-\mathrm{C}(12)-\mathrm{C}(110)$ & $171(3)$ \\
$\mathrm{C}(19)-\mathrm{C}(11)-\mathrm{C}(12)-\mathrm{C}(110)$ & $-1.0(12)$ \\
$\mathrm{S}(103)-\mathrm{C}(11)-\mathrm{C}(12)-\mathrm{C}(110)$ & $-179.8(11)$ \\
$\mathrm{C}(10)-\mathrm{C}(11)-\mathrm{C}(12)-\mathrm{C}(22)$ & $-3.8(12)$ \\
$\mathrm{C}(19)-\mathrm{C}(11)-\mathrm{C}(12)-\mathrm{C}(22)$ & $177.8(4)$ \\
$\mathrm{S}(103)-\mathrm{C}(11)-\mathrm{C}(12)-\mathrm{C}(22)$ & \\
$\mathrm{C}(10)-\mathrm{C}(11)-\mathrm{C}(12)-\mathrm{S}(3)$ & $-15)$ \\
$\mathrm{C}(19)-\mathrm{C}(11)-\mathrm{C}(12)-\mathrm{S}(3)$ & \\
$\mathrm{S}(103)-\mathrm{C}(11)-\mathrm{C}(12)-\mathrm{S}(3)$ & \\
$\mathrm{C}(9)-\mathrm{S}(3)-\mathrm{C}(12)-\mathrm{C}(11)$ & \\
& \\
& \\
&
\end{tabular}




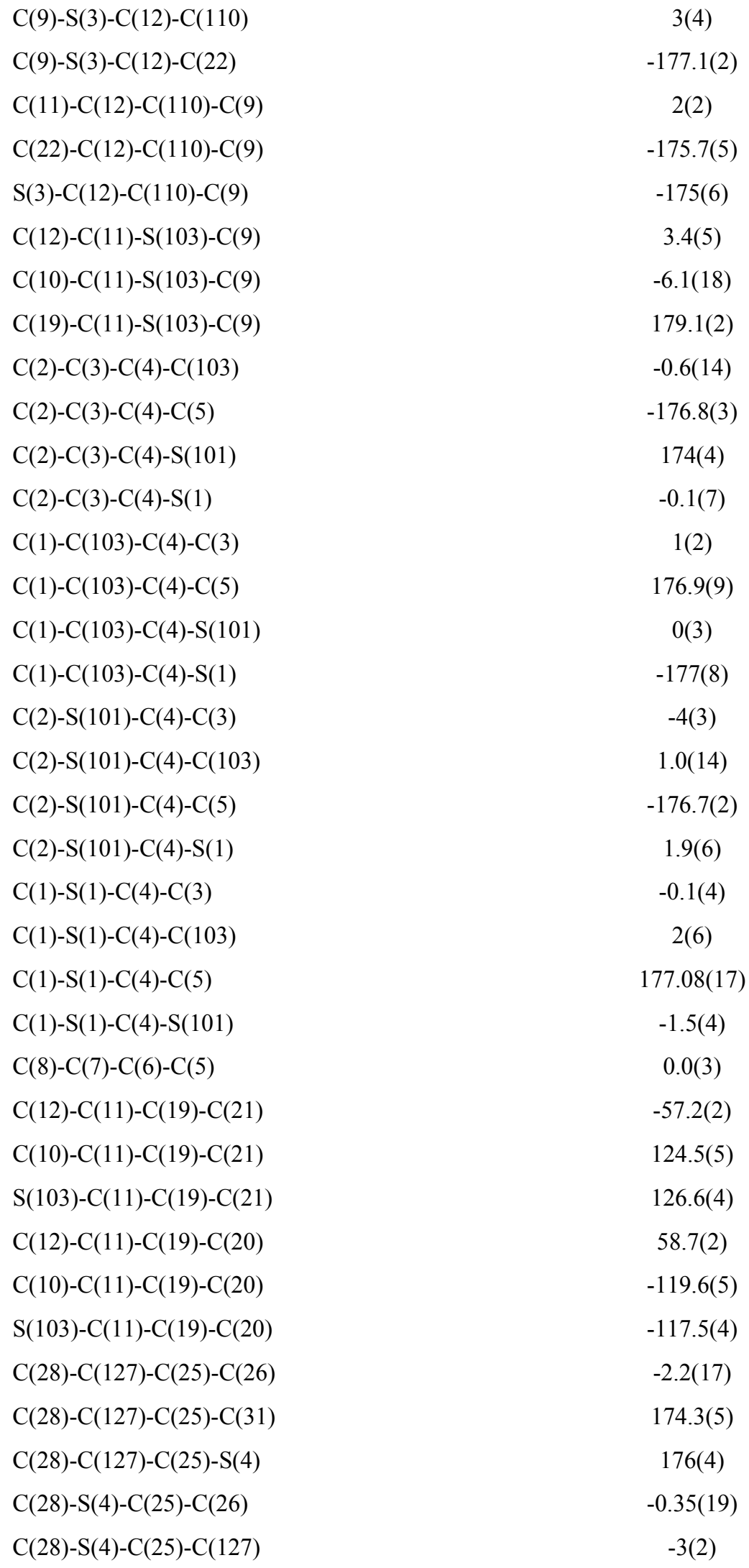




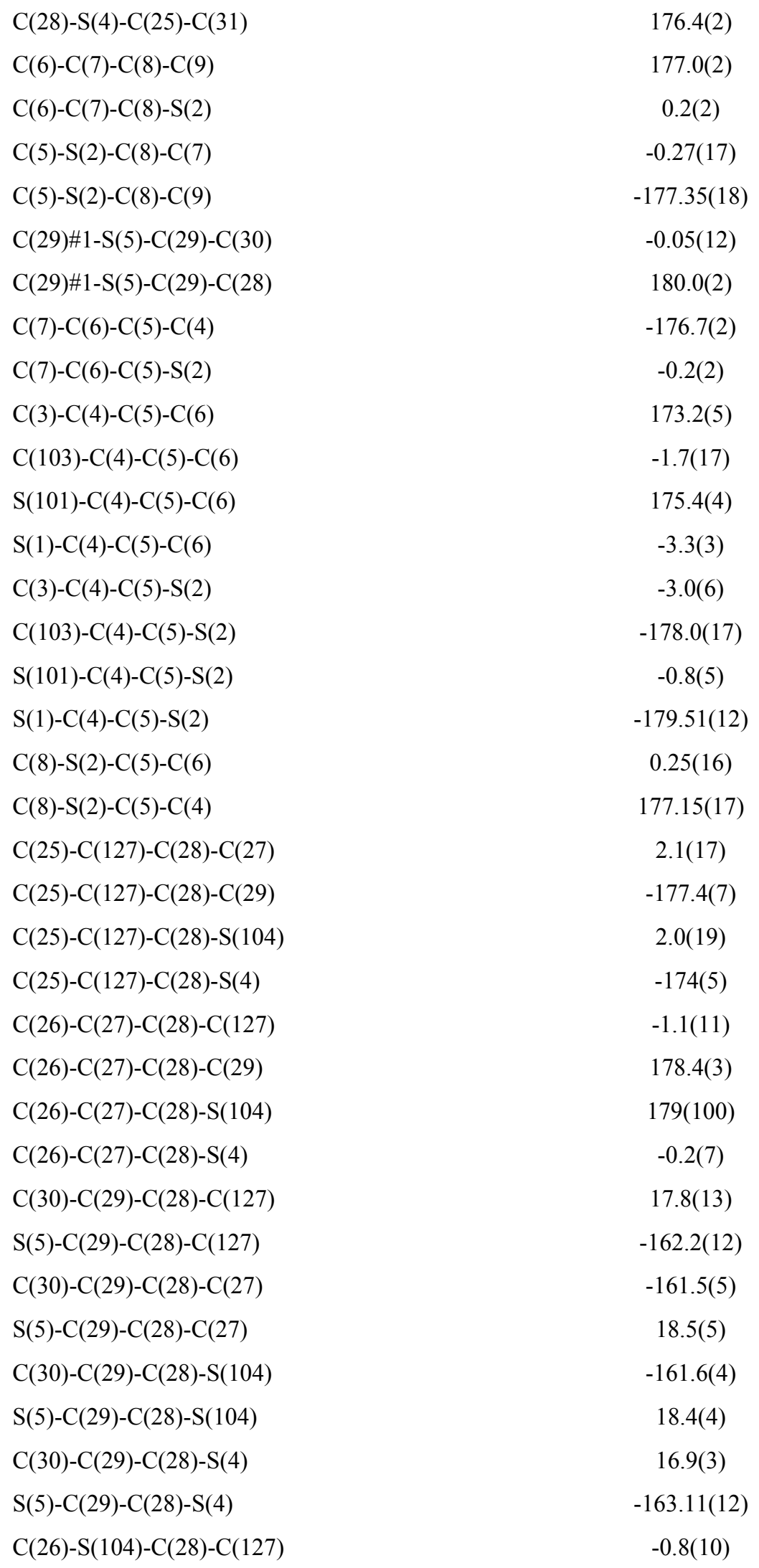




\begin{tabular}{|c|c|}
\hline$C(26)-S(104)-C(28)-C(27)$ & $-1(2)$ \\
\hline $\mathrm{C}(26)-\mathrm{S}(104)-\mathrm{C}(28)-\mathrm{C}(29)$ & $178.7(2)$ \\
\hline C(26)-S(104)-C(28)-S(4) & $0.3(4)$ \\
\hline $\mathrm{C}(25)-\mathrm{S}(4)-\mathrm{C}(28)-\mathrm{C}(127)$ & $4(4)$ \\
\hline$C(25)-S(4)-C(28)-C(27)$ & $0.3(4)$ \\
\hline C(25)-S(4)-C(28)-C(29) & $-178.45(17)$ \\
\hline $\mathrm{C}(25)-\mathrm{S}(4)-\mathrm{C}(28)-\mathrm{S}(104)$ & $0.0(3)$ \\
\hline $\mathrm{C}(11)-\mathrm{C}(10)-\mathrm{C}(9)-\mathrm{C}(110)$ & $1.7(13)$ \\
\hline $\mathrm{C}(11)-\mathrm{C}(10)-\mathrm{C}(9)-\mathrm{C}(8)$ & $178.1(3)$ \\
\hline $\mathrm{C}(11)-\mathrm{C}(10)-\mathrm{C}(9)-\mathrm{S}(3)$ & $1.0(6)$ \\
\hline $\mathrm{C}(11)-\mathrm{C}(10)-\mathrm{C}(9)-\mathrm{S}(103)$ & $-169(3)$ \\
\hline$C(12)-C(110)-C(9)-C(10)$ & $-3(2)$ \\
\hline $\mathrm{C}(12)-\mathrm{C}(110)-\mathrm{C}(9)-\mathrm{C}(8)$ & $-179.0(9)$ \\
\hline $\mathrm{C}(12)-\mathrm{C}(110)-\mathrm{C}(9)-\mathrm{S}(3)$ & $175(6)$ \\
\hline $\mathrm{C}(12)-\mathrm{C}(110)-\mathrm{C}(9)-\mathrm{S}(103)$ & $-1(2)$ \\
\hline $\mathrm{C}(7)-\mathrm{C}(8)-\mathrm{C}(9)-\mathrm{C}(10)$ & $150.9(5)$ \\
\hline $\mathrm{S}(2)-\mathrm{C}(8)-\mathrm{C}(9)-\mathrm{C}(10)$ & $-32.6(5)$ \\
\hline $\mathrm{C}(7)-\mathrm{C}(8)-\mathrm{C}(9)-\mathrm{C}(110)$ & $-33.8(15)$ \\
\hline $\mathrm{S}(2)-\mathrm{C}(8)-\mathrm{C}(9)-\mathrm{C}(110)$ & $142.7(15)$ \\
\hline $\mathrm{C}(7)-\mathrm{C}(8)-\mathrm{C}(9)-\mathrm{S}(3)$ & $-32.1(3)$ \\
\hline $\mathrm{S}(2)-\mathrm{C}(8)-\mathrm{C}(9)-\mathrm{S}(3)$ & $144.37(14)$ \\
\hline$C(7)-C(8)-C(9)-S(103)$ & $147.8(4)$ \\
\hline $\mathrm{S}(2)-\mathrm{C}(8)-\mathrm{C}(9)-\mathrm{S}(103)$ & $-35.7(4)$ \\
\hline$C(12)-S(3)-C(9)-C(10)$ & $-1.0(4)$ \\
\hline $\mathrm{C}(12)-\mathrm{S}(3)-\mathrm{C}(9)-\mathrm{C}(110)$ & $-4(5)$ \\
\hline $\mathrm{C}(12)-\mathrm{S}(3)-\mathrm{C}(9)-\mathrm{C}(8)$ & $-178.54(17)$ \\
\hline$C(12)-S(3)-C(9)-S(103)$ & $1.5(4)$ \\
\hline $\mathrm{C}(11)-\mathrm{S}(103)-\mathrm{C}(9)-\mathrm{C}(10)$ & $8(2)$ \\
\hline $\mathrm{C}(11)-\mathrm{S}(103)-\mathrm{C}(9)-\mathrm{C}(110)$ & $-1.3(12)$ \\
\hline $\mathrm{C}(11)-\mathrm{S}(103)-\mathrm{C}(9)-\mathrm{C}(8)$ & $177.4(2)$ \\
\hline $\mathrm{C}(11)-\mathrm{S}(103)-\mathrm{C}(9)-\mathrm{S}(3)$ & $-2.6(5)$ \\
\hline $\mathrm{C}(11)-\mathrm{C}(12)-\mathrm{C}(22)-\mathrm{C}(24)$ & $57.9(2)$ \\
\hline$C(110)-C(12)-C(22)-C(24)$ & $-124.1(19)$ \\
\hline $\mathrm{S}(3)-\mathrm{C}(12)-\mathrm{C}(22)-\mathrm{C}(24)$ & $-124.3(2)$ \\
\hline $\mathrm{C}(11)-\mathrm{C}(12)-\mathrm{C}(22)-\mathrm{C}(23)$ & $-57.2(2)$ \\
\hline$C(110)-C(12)-C(22)-C(23)$ & $120.8(19)$ \\
\hline
\end{tabular}




\begin{tabular}{|c|c|}
\hline$S(3)-C(12)-C(22)-C(23)$ & $120.6(2)$ \\
\hline$C(21)-C(24)-C(22)-C(12)$ & $-53.5(2)$ \\
\hline $\mathrm{C}(21)-\mathrm{C}(24)-\mathrm{C}(22)-\mathrm{C}(23)$ & $60.8(2)$ \\
\hline$C(28)-C(29)-C(30)-C(30) \# 1$ & $-179.9(2)$ \\
\hline$S(5)-C(29)-C(30)-C(30) \# 1$ & $0.1(3)$ \\
\hline $\mathrm{C}(26)-\mathrm{C}(25)-\mathrm{C}(31)-\mathrm{C}(33)$ & $-56.8(3)$ \\
\hline $\mathrm{C}(127)-\mathrm{C}(25)-\mathrm{C}(31)-\mathrm{C}(33)$ & $127.1(15)$ \\
\hline $\mathrm{S}(4)-\mathrm{C}(25)-\mathrm{C}(31)-\mathrm{C}(33)$ & $126.4(2)$ \\
\hline $\mathrm{C}(26)-\mathrm{C}(25)-\mathrm{C}(31)-\mathrm{C}(32)$ & $58.1(2)$ \\
\hline$C(127)-C(25)-C(31)-C(32)$ & $-118.0(15)$ \\
\hline $\mathrm{S}(4)-\mathrm{C}(25)-\mathrm{C}(31)-\mathrm{C}(32)$ & $-118.7(2)$ \\
\hline $\mathrm{C}(13)-\mathrm{C}(14)-\mathrm{C}(17)-\mathrm{C}(16)$ & $3.1(3)$ \\
\hline $\mathrm{C}(12)-\mathrm{C}(22)-\mathrm{C}(23)-\mathrm{C}(20)$ & $55.3(2)$ \\
\hline $\mathrm{C}(24)-\mathrm{C}(22)-\mathrm{C}(23)-\mathrm{C}(20)$ & $-59.5(2)$ \\
\hline $\mathrm{C}(26)-\mathrm{C}(34)-\mathrm{C}(35)-\mathrm{C}(32)$ & $57.6(2)$ \\
\hline$C(36)-C(34)-C(35)-C(32)$ & $-58.2(2)$ \\
\hline $\mathrm{C}(1)-\mathrm{C}(2)-\mathrm{C}(16)-\mathrm{C}(17)$ & $-57.6(3)$ \\
\hline $\mathrm{C}(3)-\mathrm{C}(2)-\mathrm{C}(16)-\mathrm{C}(17)$ & $126.9(6)$ \\
\hline$S(101)-C(2)-C(16)-C(17)$ & $124.2(5)$ \\
\hline $\mathrm{C}(1)-\mathrm{C}(2)-\mathrm{C}(16)-\mathrm{C}(18)$ & $57.9(3)$ \\
\hline$C(3)-C(2)-C(16)-C(18)$ & $-117.6(6)$ \\
\hline $\mathrm{S}(101)-\mathrm{C}(2)-\mathrm{C}(16)-\mathrm{C}(18)$ & $-120.3(5)$ \\
\hline $\mathrm{C}(14)-\mathrm{C}(17)-\mathrm{C}(16)-\mathrm{C}(2)$ & $52.7(2)$ \\
\hline $\mathrm{C}(14)-\mathrm{C}(17)-\mathrm{C}(16)-\mathrm{C}(18)$ & $-62.3(2)$ \\
\hline$C(127)-C(25)-C(26)-C(27)$ & $1.1(9)$ \\
\hline$C(31)-C(25)-C(26)-C(27)$ & $-176.9(4)$ \\
\hline $\mathrm{S}(4)-\mathrm{C}(25)-\mathrm{C}(26)-\mathrm{C}(27)$ & $0.3(4)$ \\
\hline $\mathrm{C}(127)-\mathrm{C}(25)-\mathrm{C}(26)-\mathrm{C}(34)$ & $176.6(8)$ \\
\hline$C(31)-C(25)-C(26)-C(34)$ & $-1.4(3)$ \\
\hline$S(4)-C(25)-C(26)-C(34)$ & $175.86(15)$ \\
\hline$C(127)-C(25)-C(26)-S(104)$ & $1.5(9)$ \\
\hline $\mathrm{C}(31)-\mathrm{C}(25)-\mathrm{C}(26)-\mathrm{S}(104)$ & $-176.5(4)$ \\
\hline$S(4)-C(25)-C(26)-S(104)$ & $0.7(4)$ \\
\hline $\mathrm{C}(28)-\mathrm{C}(27)-\mathrm{C}(26)-\mathrm{C}(25)$ & $-0.1(7)$ \\
\hline $\mathrm{C}(28)-\mathrm{C}(27)-\mathrm{C}(26)-\mathrm{C}(34)$ & $-174.1(3)$ \\
\hline $\mathrm{C}(28)-\mathrm{C}(27)-\mathrm{C}(26)-\mathrm{S}(104)$ & $-179(100)$ \\
\hline
\end{tabular}




\begin{tabular}{|c|c|}
\hline$C(36)-C(34)-C(26)-C(25)$ & $57.8(2)$ \\
\hline$C(35)-C(34)-C(26)-C(25)$ & $-57.7(2)$ \\
\hline$C(36)-C(34)-C(26)-C(27)$ & $-128.3(5)$ \\
\hline$C(35)-C(34)-C(26)-C(27)$ & $116.2(5)$ \\
\hline$C(36)-C(34)-C(26)-S(104)$ & $-126.9(4)$ \\
\hline$C(35)-C(34)-C(26)-S(104)$ & $117.7(4)$ \\
\hline $\mathrm{C}(28)-\mathrm{S}(104)-\mathrm{C}(26)-\mathrm{C}(25)$ & $-0.6(5)$ \\
\hline $\mathrm{C}(28)-\mathrm{S}(104)-\mathrm{C}(26)-\mathrm{C}(27)$ & $0.8(18)$ \\
\hline $\mathrm{C}(28)-\mathrm{S}(104)-\mathrm{C}(26)-\mathrm{C}(34)$ & $-175.4(2)$ \\
\hline$C(11)-C(19)-C(20)-C(23)$ & $-54.9(2)$ \\
\hline$C(21)-C(19)-C(20)-C(23)$ & $60.7(2)$ \\
\hline $\mathrm{C}(22)-\mathrm{C}(23)-\mathrm{C}(20)-\mathrm{C}(19)$ & $-1.0(3)$ \\
\hline $\mathrm{C}(11)-\mathrm{C}(19)-\mathrm{C}(21)-\mathrm{C}(24)$ & $56.0(2)$ \\
\hline $\mathrm{C}(20)-\mathrm{C}(19)-\mathrm{C}(21)-\mathrm{C}(24)$ & $-59.3(2)$ \\
\hline $\mathrm{C}(22)-\mathrm{C}(24)-\mathrm{C}(21)-\mathrm{C}(19)$ & $-1.4(2)$ \\
\hline$C(26)-C(34)-C(36)-C(33)$ & $-52.6(2)$ \\
\hline $\mathrm{C}(35)-\mathrm{C}(34)-\mathrm{C}(36)-\mathrm{C}(33)$ & $62.1(2)$ \\
\hline$C(2)-C(1)-C(13)-C(14)$ & $56.9(3)$ \\
\hline$C(103)-C(1)-C(13)-C(14)$ & $-127(2)$ \\
\hline $\mathrm{S}(1)-\mathrm{C}(1)-\mathrm{C}(13)-\mathrm{C}(14)$ & $-125.3(2)$ \\
\hline $\mathrm{C}(2)-\mathrm{C}(1)-\mathrm{C}(13)-\mathrm{C}(15)$ & $-57.8(3)$ \\
\hline$C(103)-C(1)-C(13)-C(15)$ & $118(2)$ \\
\hline$S(1)-C(1)-C(13)-C(15)$ & $120.0(2)$ \\
\hline$C(17)-C(14)-C(13)-C(1)$ & $-56.2(2)$ \\
\hline$C(17)-C(14)-C(13)-C(15)$ & $57.8(2)$ \\
\hline $\mathrm{C}(18)-\mathrm{C}(15)-\mathrm{C}(13)-\mathrm{C}(1)$ & $52.9(2)$ \\
\hline$C(18)-C(15)-C(13)-C(14)$ & $-61.3(2)$ \\
\hline$C(2)-C(16)-C(18)-C(15)$ & $-57.2(2)$ \\
\hline$C(17)-C(16)-C(18)-C(15)$ & $58.8(2)$ \\
\hline$C(13)-C(15)-C(18)-C(16)$ & $2.4(3)$ \\
\hline$C(34)-C(35)-C(32)-C(31)$ & $-3.2(3)$ \\
\hline$C(25)-C(31)-C(32)-C(35)$ & $-52.4(2)$ \\
\hline $\mathrm{C}(33)-\mathrm{C}(31)-\mathrm{C}(32)-\mathrm{C}(35)$ & $61.9(2)$ \\
\hline $\mathrm{C}(25)-\mathrm{C}(31)-\mathrm{C}(33)-\mathrm{C}(36)$ & $56.4(3)$ \\
\hline $\mathrm{C}(32)-\mathrm{C}(31)-\mathrm{C}(33)-\mathrm{C}(36)$ & $-58.0(3)$ \\
\hline $\mathrm{C}(34)-\mathrm{C}(36)-\mathrm{C}(33)-\mathrm{C}(31)$ & $-3.3(3)$ \\
\hline
\end{tabular}


Symmetry transformations used to generate equivalent atoms: $\# 1-\mathrm{x}+1, \mathrm{y},-\mathrm{z}+3 / 2$ 
X-Ray Crystallography of $2^{\circ+} \mathrm{SbF}_{6}^{-}(100 \mathrm{~K})$.
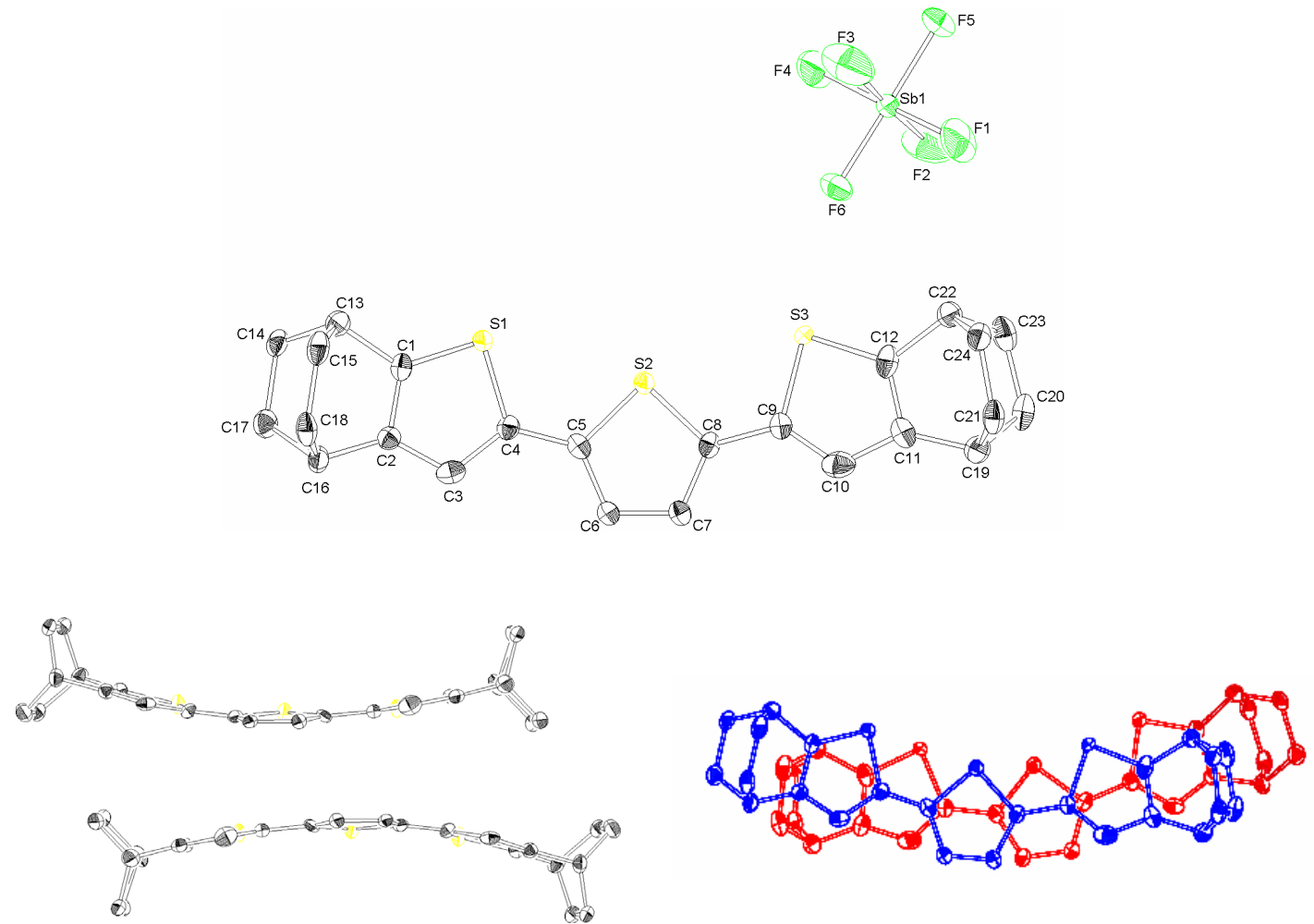

\section{Packing Structure}

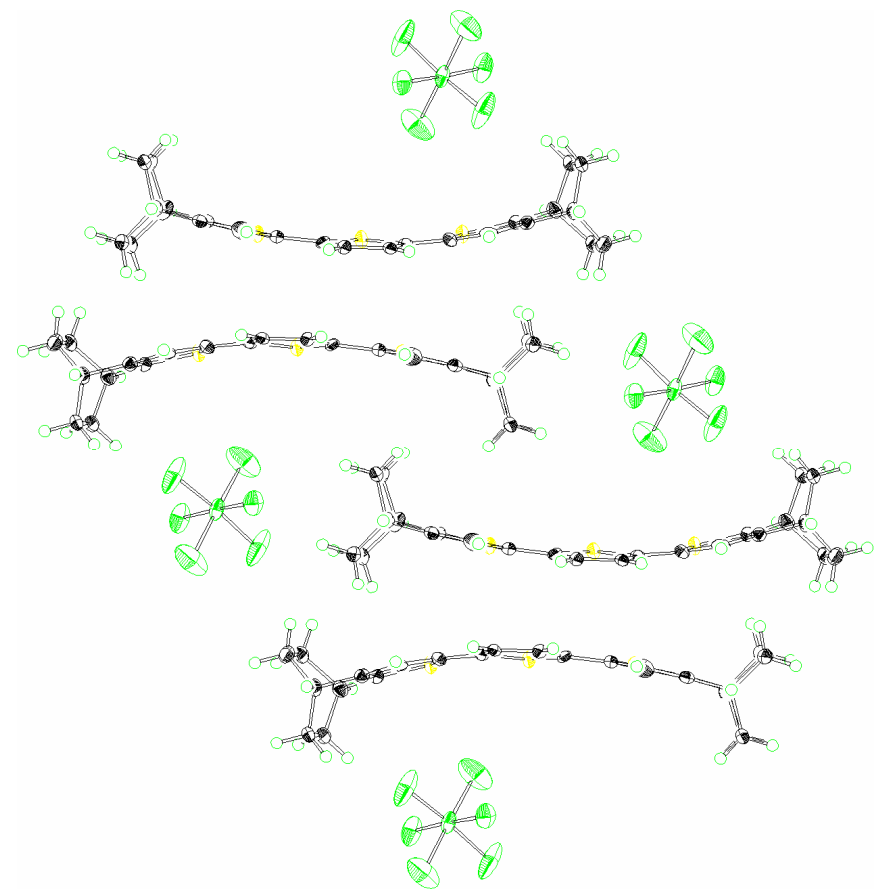


Table 1. Crystal data and structure refinement for ${ }^{\cdot+} \mathbf{S b F}_{6}{ }^{-}$.

Identification code

Empirical formula

Formula weight

Temperature

Wavelength

Crystal system

Space group

Unit cell dimensions

Volume

Z

Density (calculated)

Absorption coefficient

$\mathrm{F}(000)$

Crystal size

Theta range for data collection

Index ranges

Reflections collected

Independent reflections

Completeness to theta $=25.00^{\circ}$

Absorption correction

Max. and min. transmission

Refinement method

Data / restraints / parameters

Goodness-of-fit on $\mathrm{F}^{2}$

Final $\mathrm{R}$ indices $[\mathrm{I}>2 \operatorname{sigma}(\mathrm{I})]$

$\mathrm{R}$ indices (all data)

Largest diff. peak and hole
$100 \mathrm{KpD}$

C24 H24 F6 S3 Sb

644.36

$100(2) \mathrm{K}$

$0.71073 \AA$

Orthorhombic

Pben

$\begin{array}{ll}\mathrm{a}=11.6808(17) \AA & \alpha=90^{\circ} . \\ \mathrm{b}=21.565(3) \AA & \beta=90^{\circ} . \\ \mathrm{c}=19.163(3) \AA & \gamma=90^{\circ} .\end{array}$

$4826.9(12) \AA^{3}$

8

$1.773 \mathrm{Mg} / \mathrm{m}^{3}$

$1.461 \mathrm{~mm}^{-1}$

2568

$0.15 \times 0.15 \times 0.10 \mathrm{~mm}^{3}$

1.89 to $25.00^{\circ}$.

$-13<=\mathrm{h}<=13,-25<=\mathrm{k}<=20,-22<=1<=22$

23249

$4244[\mathrm{R}(\mathrm{int})=0.0586]$

$99.9 \%$

Empirical

0.8677 and 0.8106

Full-matrix least-squares on $\mathrm{F}^{2}$

4244 / 12 / 340

1.002

$\mathrm{R} 1=0.0490, \mathrm{wR} 2=0.1004$

$\mathrm{R} 1=0.0784, \mathrm{wR} 2=0.1092$

1.741 and -0.444 e. $\AA^{-3}$ 
Table 2. Atomic coordinates $\left(\mathrm{x} 10^{4}\right)$ and equivalent isotropic displacement parameters $\left(\AA^{2} \times 10^{3}\right)$ for $\mathbf{2}^{\cdot+} \mathbf{S b F}_{6}^{-}$. $\quad U(\mathrm{eq})$ is defined as one third of the trace of the orthogonalized $\mathrm{U}^{\mathrm{ij}}$ tensor.

\begin{tabular}{|c|c|c|c|c|}
\hline & $\mathrm{x}$ & $\mathrm{y}$ & z & $\mathrm{U}(\mathrm{eq})$ \\
\hline $\mathrm{C}(18)$ & 11211(5) & $6253(3)$ & 6881(3) & $28(1)$ \\
\hline$C(12)$ & $6647(4)$ & $6237(3)$ & $1628(3)$ & $27(1)$ \\
\hline$C(17)$ & $13196(5)$ & $6158(3)$ & $6410(3)$ & $27(1)$ \\
\hline$C(13)$ & $11702(5)$ & $5353(3)$ & $6085(3)$ & $25(1)$ \\
\hline $\mathrm{C}(11)$ & $6561(4)$ & $6879(2)$ & $1569(3)$ & $25(1)$ \\
\hline$C(15)$ & $10987(5)$ & $5554(3)$ & $6725(3)$ & $29(1)$ \\
\hline$C(16)$ & $12064(5)$ & $6528(3)$ & $6351(3)$ & $25(1)$ \\
\hline$C(20)$ & $5793(5)$ & $6816(3)$ & $388(3)$ & $30(1)$ \\
\hline$C(22)$ & $5760(5)$ & $5888(3)$ & $1203(3)$ & $29(1)$ \\
\hline$C(7)$ & $9334(4)$ & $7358(2)$ & $3162(3)$ & $20(1)$ \\
\hline$C(9)$ & $8052(4)$ & $6694(2)$ & $2385(3)$ & $20(1)$ \\
\hline$C(2)$ & $11589(4)$ & $6410(2)$ & $5653(3)$ & $20(1)$ \\
\hline$C(23)$ & $5935(5)$ & $6110(3)$ & $445(3)$ & $33(1)$ \\
\hline$C(6)$ & $10052(4)$ & $7287(2)$ & $3723(3)$ & $18(1)$ \\
\hline C(19) & $5614(5)$ & 7101(3) & $1119(3)$ & $27(1)$ \\
\hline$C(8)$ & $8870(4)$ & $6790(2)$ & 2919(3) & $19(1)$ \\
\hline$C(14)$ & $12988(5)$ & $5462(2)$ & $6248(3)$ & $24(1)$ \\
\hline$C(24)$ & $4562(5)$ & $6118(2)$ & $1454(3)$ & $27(1)$ \\
\hline$C(1)$ & $11378(5)$ & $5798(2)$ & $5496(3)$ & $22(1)$ \\
\hline$C(5)$ & $10176(5)$ & $6670(2)$ & $3939(3)$ & $20(1)$ \\
\hline$C(21)$ & $4494(5)$ & $6835(3)$ & $1421(3)$ & $28(1)$ \\
\hline$C(4)$ & $10760(5)$ & $6436(2)$ & $4527(3)$ & $21(1)$ \\
\hline $\mathrm{Sb}(1)$ & $7261(1)$ & $3978(1)$ & $942(1)$ & $30(1)$ \\
\hline $\mathrm{S}(2)$ & $9380(1)$ & $6178(1)$ & $3411(1)$ & $21(1)$ \\
\hline$F(6)$ & $7676(3)$ & $4641(2)$ & $1512(2)$ & $51(1)$ \\
\hline$F(5)$ & $6803(3)$ & $3298(2)$ & $400(2)$ & $44(1)$ \\
\hline $\mathrm{F}(1)$ & $5850(4)$ & $4357(2)$ & $795(3)$ & $84(2)$ \\
\hline $\mathrm{F}(4)$ & $8607(4)$ & $3582(2)$ & $1153(3)$ & $79(2)$ \\
\hline $\mathrm{F}(2)$ & $7859(5)$ & $4355(2)$ & $173(2)$ & $86(2)$ \\
\hline $\mathrm{F}(3)$ & $6595(5)$ & $3580(2)$ & $1724(2)$ & $87(2)$ \\
\hline$C(10)$ & $7407(12)$ & $7092(5)$ & $2023(7)$ & $34(4)$ \\
\hline
\end{tabular}




\begin{tabular}{lllll}
$\mathrm{S}(3)$ & $7670(3)$ & $5921(1)$ & $2148(2)$ & $18(1)$ \\
$\mathrm{S}(13)$ & $7429(6)$ & $7374(3)$ & $1998(4)$ & $26(2)$ \\
$\mathrm{C}(110)$ & $7531(17)$ & $6181(6)$ & $2131(12)$ & $26(7)$ \\
$\mathrm{S}(1)$ & $10797(2)$ & $5647(1)$ & $4712(1)$ & $21(1)$ \\
$\mathrm{C}(3)$ & $11244(16)$ & $6770(5)$ & $5084(6)$ & $23(2)$ \\
$\mathrm{C}(103)$ & $10810(100)$ & $5829(8)$ & $4830(30)$ & $70(60)$ \\
$\mathrm{S}(11)$ & $11220(40)$ & $6977(4)$ & $5125(12)$ & $26(7)$ \\
\hline
\end{tabular}


Table 3. Bond lengths $[\AA]$ and angles $\left[{ }^{\circ}\right]$ for $\mathbf{2}^{\mathbf{}} \mathbf{S b F}_{\mathbf{6}}{ }^{-}$.

\begin{tabular}{|c|c|}
\hline $\mathrm{C}(18)-\mathrm{C}(16)$ & $1.542(8)$ \\
\hline $\mathrm{C}(18)-\mathrm{C}(15)$ & $1.559(8)$ \\
\hline $\mathrm{C}(18)-\mathrm{H}(18 \mathrm{~A})$ & 0.9900 \\
\hline $\mathrm{C}(18)-\mathrm{H}(18 \mathrm{~B})$ & 0.9900 \\
\hline $\mathrm{C}(12)-\mathrm{C}(11)$ & $1.391(8)$ \\
\hline $\mathrm{C}(12)-\mathrm{C}(110)$ & $1.417(15)$ \\
\hline $\mathrm{C}(12)-\mathrm{C}(22)$ & $1.518(7)$ \\
\hline$C(12)-S(3)$ & $1.699(5)$ \\
\hline $\mathrm{C}(17)-\mathrm{C}(16)$ & $1.549(8)$ \\
\hline $\mathrm{C}(17)-\mathrm{C}(14)$ & $1.553(7)$ \\
\hline $\mathrm{C}(17)-\mathrm{H}(17 \mathrm{~A})$ & 0.9900 \\
\hline $\mathrm{C}(17)-\mathrm{H}(17 \mathrm{~B})$ & 0.9900 \\
\hline $\mathrm{C}(13)-\mathrm{C}(1)$ & $1.528(7)$ \\
\hline$C(13)-C(15)$ & $1.546(8)$ \\
\hline$C(13)-C(14)$ & $1.552(8)$ \\
\hline $\mathrm{C}(13)-\mathrm{H}(13)$ & 1.0000 \\
\hline $\mathrm{C}(11)-\mathrm{C}(10)$ & $1.395(13)$ \\
\hline$C(11)-C(19)$ & $1.483(7)$ \\
\hline $\mathrm{C}(11)-\mathrm{S}(13)$ & $1.687(7)$ \\
\hline $\mathrm{C}(15)-\mathrm{H}(15 \mathrm{~A})$ & 0.9900 \\
\hline $\mathrm{C}(15)-\mathrm{H}(15 \mathrm{~B})$ & 0.9900 \\
\hline$C(16)-C(2)$ & $1.470(7)$ \\
\hline $\mathrm{C}(16)-\mathrm{H}(16)$ & 1.0000 \\
\hline $\mathrm{C}(20)-\mathrm{C}(23)$ & $1.536(8)$ \\
\hline$C(20)-C(19)$ & $1.544(7)$ \\
\hline $\mathrm{C}(20)-\mathrm{H}(20 \mathrm{~A})$ & 0.9900 \\
\hline $\mathrm{C}(20)-\mathrm{H}(20 \mathrm{~B})$ & 0.9900 \\
\hline$C(22)-C(23)$ & $1.543(8)$ \\
\hline$C(22)-C(24)$ & $1.561(8)$ \\
\hline $\mathrm{C}(22)-\mathrm{H}(22)$ & 1.0000 \\
\hline$C(7)-C(6)$ & $1.372(7)$ \\
\hline$C(7)-C(8)$ & $1.419(7)$ \\
\hline $\mathrm{C}(7)-\mathrm{H}(7)$ & 0.9500 \\
\hline$C(9)-C(10)$ & $1.337(13)$ \\
\hline
\end{tabular}




\begin{tabular}{|c|c|}
\hline $\mathrm{C}(9)-\mathrm{C}(110)$ & $1.353(15)$ \\
\hline $\mathrm{C}(9)-\mathrm{C}(8)$ & $1.415(7)$ \\
\hline $\mathrm{C}(9)-\mathrm{S}(3)$ & $1.784(5)$ \\
\hline$C(9)-S(13)$ & $1.797(6)$ \\
\hline$C(2)-C(1)$ & $1.375(7)$ \\
\hline$C(2)-C(3)$ & $1.399(11)$ \\
\hline$C(2)-S(11)$ & $1.646(10)$ \\
\hline $\mathrm{C}(23)-\mathrm{H}(23 \mathrm{~A})$ & 0.9900 \\
\hline $\mathrm{C}(23)-\mathrm{H}(23 \mathrm{~B})$ & 0.9900 \\
\hline$C(6)-C(5)$ & $1.401(7)$ \\
\hline $\mathrm{C}(6)-\mathrm{H}(6)$ & 0.9500 \\
\hline$C(19)-C(21)$ & $1.541(8)$ \\
\hline C(19)-H(19) & 1.0000 \\
\hline $\mathrm{C}(8)-\mathrm{S}(2)$ & $1.727(5)$ \\
\hline $\mathrm{C}(14)-\mathrm{H}(14 \mathrm{~A})$ & 0.9900 \\
\hline $\mathrm{C}(14)-\mathrm{H}(14 \mathrm{~B})$ & 0.9900 \\
\hline $\mathrm{C}(24)-\mathrm{C}(21)$ & $1.549(8)$ \\
\hline $\mathrm{C}(24)-\mathrm{H}(24 \mathrm{~A})$ & 0.9900 \\
\hline $\mathrm{C}(24)-\mathrm{H}(24 \mathrm{~B})$ & 0.9900 \\
\hline $\mathrm{C}(1)-\mathrm{C}(103)$ & $1.443(17)$ \\
\hline $\mathrm{C}(1)-\mathrm{S}(1)$ & $1.682(5)$ \\
\hline$C(5)-C(4)$ & $1.411(7)$ \\
\hline$C(5)-S(2)$ & $1.736(5)$ \\
\hline $\mathrm{C}(21)-\mathrm{H}(21 \mathrm{~A})$ & 0.9900 \\
\hline $\mathrm{C}(21)-\mathrm{H}(21 \mathrm{~B})$ & 0.9900 \\
\hline$C(4)-C(3)$ & $1.405(12)$ \\
\hline$C(4)-C(103)$ & $1.433(18)$ \\
\hline$C(4)-S(11)$ & $1.720(11)$ \\
\hline$C(4)-S(1)$ & $1.738(5)$ \\
\hline $\mathrm{Sb}(1)-\mathrm{F}(2)$ & $1.821(4)$ \\
\hline $\mathrm{Sb}(1)-\mathrm{F}(4)$ & $1.834(4)$ \\
\hline $\mathrm{Sb}(1)-\mathrm{F}(1)$ & $1.861(4)$ \\
\hline $\mathrm{Sb}(1)-\mathrm{F}(6)$ & $1.864(3)$ \\
\hline $\mathrm{Sb}(1)-\mathrm{F}(5)$ & $1.876(3)$ \\
\hline $\mathrm{Sb}(1)-\mathrm{F}(3)$ & $1.894(4)$ \\
\hline $\mathrm{C}(10)-\mathrm{H}(10)$ & 0.9500 \\
\hline
\end{tabular}




\begin{tabular}{|c|c|}
\hline $\mathrm{C}(110)-\mathrm{H}(110)$ & 0.9500 \\
\hline $\mathrm{C}(3)-\mathrm{H}(3)$ & 0.9500 \\
\hline $\mathrm{C}(103)-\mathrm{H}(103)$ & 0.9500 \\
\hline$C(16)-C(18)-C(15)$ & $110.8(4)$ \\
\hline $\mathrm{C}(16)-\mathrm{C}(18)-\mathrm{H}(18 \mathrm{~A})$ & 109.5 \\
\hline $\mathrm{C}(15)-\mathrm{C}(18)-\mathrm{H}(18 \mathrm{~A})$ & 109.5 \\
\hline $\mathrm{C}(16)-\mathrm{C}(18)-\mathrm{H}(18 \mathrm{~B})$ & 109.5 \\
\hline $\mathrm{C}(15)-\mathrm{C}(18)-\mathrm{H}(18 \mathrm{~B})$ & 109.5 \\
\hline $\mathrm{H}(18 \mathrm{~A})-\mathrm{C}(18)-\mathrm{H}(18 \mathrm{~B})$ & 108.1 \\
\hline $\mathrm{C}(11)-\mathrm{C}(12)-\mathrm{C}(110)$ & $101.1(6)$ \\
\hline$C(11)-C(12)-C(22)$ & $113.6(4)$ \\
\hline $\mathrm{C}(110)-\mathrm{C}(12)-\mathrm{C}(22)$ & $145.0(7)$ \\
\hline$C(11)-C(12)-S(3)$ & $119.8(3)$ \\
\hline$C(110)-C(12)-S(3)$ & $19.0(6)$ \\
\hline $\mathrm{C}(22)-\mathrm{C}(12)-\mathrm{S}(3)$ & $126.5(4)$ \\
\hline$C(16)-C(17)-C(14)$ & $110.5(4)$ \\
\hline $\mathrm{C}(16)-\mathrm{C}(17)-\mathrm{H}(17 \mathrm{~A})$ & 109.6 \\
\hline $\mathrm{C}(14)-\mathrm{C}(17)-\mathrm{H}(17 \mathrm{~A})$ & 109.5 \\
\hline $\mathrm{C}(16)-\mathrm{C}(17)-\mathrm{H}(17 \mathrm{~B})$ & 109.6 \\
\hline $\mathrm{C}(14)-\mathrm{C}(17)-\mathrm{H}(17 \mathrm{~B})$ & 109.6 \\
\hline $\mathrm{H}(17 \mathrm{~A})-\mathrm{C}(17)-\mathrm{H}(17 \mathrm{~B})$ & 108.1 \\
\hline$C(1)-C(13)-C(15)$ & $106.1(4)$ \\
\hline$C(1)-C(13)-C(14)$ & $107.1(4)$ \\
\hline$C(15)-C(13)-C(14)$ & $108.7(4)$ \\
\hline $\mathrm{C}(1)-\mathrm{C}(13)-\mathrm{H}(13)$ & 111.6 \\
\hline$C(15)-C(13)-H(13)$ & 111.6 \\
\hline $\mathrm{C}(14)-\mathrm{C}(13)-\mathrm{H}(13)$ & 111.6 \\
\hline$C(12)-C(11)-C(10)$ & $103.1(5)$ \\
\hline$C(12)-C(11)-C(19)$ & $115.0(4)$ \\
\hline$C(10)-C(11)-C(19)$ & $141.7(6)$ \\
\hline $\mathrm{C}(12)-\mathrm{C}(11)-\mathrm{S}(13)$ & $123.1(4)$ \\
\hline$C(10)-C(11)-S(13)$ & $20.1(5)$ \\
\hline$C(19)-C(11)-S(13)$ & $121.8(4)$ \\
\hline$C(13)-C(15)-C(18)$ & $109.4(4)$ \\
\hline $\mathrm{C}(13)-\mathrm{C}(15)-\mathrm{H}(15 \mathrm{~A})$ & 109.8 \\
\hline
\end{tabular}




\begin{tabular}{|c|c|}
\hline $\mathrm{C}(18)-\mathrm{C}(15)-\mathrm{H}(15 \mathrm{~A})$ & 109.8 \\
\hline $\mathrm{C}(13)-\mathrm{C}(15)-\mathrm{H}(15 \mathrm{~B})$ & 109.8 \\
\hline $\mathrm{C}(18)-\mathrm{C}(15)-\mathrm{H}(15 \mathrm{~B})$ & 109.8 \\
\hline $\mathrm{H}(15 \mathrm{~A})-\mathrm{C}(15)-\mathrm{H}(15 \mathrm{~B})$ & 108.2 \\
\hline $\mathrm{C}(2)-\mathrm{C}(16)-\mathrm{C}(18)$ & $106.7(4)$ \\
\hline$C(2)-C(16)-C(17)$ & $107.5(5)$ \\
\hline $\mathrm{C}(18)-\mathrm{C}(16)-\mathrm{C}(17)$ & $107.8(5)$ \\
\hline$C(2)-C(16)-H(16)$ & 111.6 \\
\hline $\mathrm{C}(18)-\mathrm{C}(16)-\mathrm{H}(16)$ & 111.6 \\
\hline $\mathrm{C}(17)-\mathrm{C}(16)-\mathrm{H}(16)$ & 111.5 \\
\hline$C(23)-C(20)-C(19)$ & $110.2(4)$ \\
\hline $\mathrm{C}(23)-\mathrm{C}(20)-\mathrm{H}(20 \mathrm{~A})$ & 109.6 \\
\hline $\mathrm{C}(19)-\mathrm{C}(20)-\mathrm{H}(20 \mathrm{~A})$ & 109.6 \\
\hline $\mathrm{C}(23)-\mathrm{C}(20)-\mathrm{H}(20 \mathrm{~B})$ & 109.6 \\
\hline $\mathrm{C}(19)-\mathrm{C}(20)-\mathrm{H}(20 \mathrm{~B})$ & 109.6 \\
\hline $\mathrm{H}(20 \mathrm{~A})-\mathrm{C}(20)-\mathrm{H}(20 \mathrm{~B})$ & 108.1 \\
\hline $\mathrm{C}(12)-\mathrm{C}(22)-\mathrm{C}(23)$ & $105.1(5)$ \\
\hline$C(12)-C(22)-C(24)$ & $106.8(5)$ \\
\hline$C(23)-C(22)-C(24)$ & $108.1(5)$ \\
\hline $\mathrm{C}(12)-\mathrm{C}(22)-\mathrm{H}(22)$ & 112.2 \\
\hline $\mathrm{C}(23)-\mathrm{C}(22)-\mathrm{H}(22)$ & 112.2 \\
\hline $\mathrm{C}(24)-\mathrm{C}(22)-\mathrm{H}(22)$ & 112.2 \\
\hline$C(6)-C(7)-C(8)$ & $113.3(5)$ \\
\hline $\mathrm{C}(6)-\mathrm{C}(7)-\mathrm{H}(7)$ & 123.4 \\
\hline $\mathrm{C}(8)-\mathrm{C}(7)-\mathrm{H}(7)$ & 123.4 \\
\hline$C(10)-C(9)-C(110)$ & $94.8(10)$ \\
\hline$C(10)-C(9)-C(8)$ & $131.4(6)$ \\
\hline$C(110)-C(9)-C(8)$ & $133.1(7)$ \\
\hline $\mathrm{C}(10)-\mathrm{C}(9)-\mathrm{S}(3)$ & 109.1(6) \\
\hline$C(110)-C(9)-S(3)$ & $14.6(6)$ \\
\hline$C(8)-C(9)-S(3)$ & 119.3(4) \\
\hline$C(10)-C(9)-S(13)$ & $14.8(5)$ \\
\hline $\mathrm{C}(110)-\mathrm{C}(9)-\mathrm{S}(13)$ & $109.6(6)$ \\
\hline $\mathrm{C}(8)-\mathrm{C}(9)-\mathrm{S}(13)$ & $116.9(4)$ \\
\hline$S(3)-C(9)-S(13)$ & $123.8(4)$ \\
\hline$C(1)-C(2)-C(3)$ & $108.1(6)$ \\
\hline
\end{tabular}




\begin{tabular}{|c|c|}
\hline$C(1)-C(2)-C(16)$ & $115.7(4)$ \\
\hline$C(3)-C(2)-C(16)$ & $136.2(6)$ \\
\hline $\mathrm{C}(1)-\mathrm{C}(2)-\mathrm{S}(11)$ & $122.1(5)$ \\
\hline $\mathrm{C}(3)-\mathrm{C}(2)-\mathrm{S}(11)$ & $14.5(8)$ \\
\hline$C(16)-C(2)-S(11)$ & $122.0(6)$ \\
\hline$C(20)-C(23)-C(22)$ & $111.1(5)$ \\
\hline $\mathrm{C}(20)-\mathrm{C}(23)-\mathrm{H}(23 \mathrm{~A})$ & 109.4 \\
\hline $\mathrm{C}(22)-\mathrm{C}(23)-\mathrm{H}(23 \mathrm{~A})$ & 109.4 \\
\hline $\mathrm{C}(20)-\mathrm{C}(23)-\mathrm{H}(23 \mathrm{~B})$ & 109.4 \\
\hline $\mathrm{C}(22)-\mathrm{C}(23)-\mathrm{H}(23 \mathrm{~B})$ & 109.4 \\
\hline $\mathrm{H}(23 \mathrm{~A})-\mathrm{C}(23)-\mathrm{H}(23 \mathrm{~B})$ & 108.0 \\
\hline$C(7)-C(6)-C(5)$ & $113.6(5)$ \\
\hline $\mathrm{C}(7)-\mathrm{C}(6)-\mathrm{H}(6)$ & 123.2 \\
\hline $\mathrm{C}(5)-\mathrm{C}(6)-\mathrm{H}(6)$ & 123.2 \\
\hline$C(11)-C(19)-C(21)$ & $107.2(4)$ \\
\hline$C(11)-C(19)-C(20)$ & $107.3(5)$ \\
\hline$C(21)-C(19)-C(20)$ & $107.8(5)$ \\
\hline $\mathrm{C}(11)-\mathrm{C}(19)-\mathrm{H}(19)$ & 111.5 \\
\hline $\mathrm{C}(21)-\mathrm{C}(19)-\mathrm{H}(19)$ & 111.4 \\
\hline $\mathrm{C}(20)-\mathrm{C}(19)-\mathrm{H}(19)$ & 111.5 \\
\hline $\mathrm{C}(9)-\mathrm{C}(8)-\mathrm{C}(7)$ & $128.4(5)$ \\
\hline$C(9)-C(8)-S(2)$ & $121.1(4)$ \\
\hline$C(7)-C(8)-S(2)$ & $110.4(4)$ \\
\hline$C(13)-C(14)-C(17)$ & $109.8(4)$ \\
\hline $\mathrm{C}(13)-\mathrm{C}(14)-\mathrm{H}(14 \mathrm{~A})$ & 109.7 \\
\hline $\mathrm{C}(17)-\mathrm{C}(14)-\mathrm{H}(14 \mathrm{~A})$ & 109.7 \\
\hline $\mathrm{C}(13)-\mathrm{C}(14)-\mathrm{H}(14 \mathrm{~B})$ & 109.7 \\
\hline $\mathrm{C}(17)-\mathrm{C}(14)-\mathrm{H}(14 \mathrm{~B})$ & 109.7 \\
\hline $\mathrm{H}(14 \mathrm{~A})-\mathrm{C}(14)-\mathrm{H}(14 \mathrm{~B})$ & 108.2 \\
\hline$C(21)-C(24)-C(22)$ & $110.5(4)$ \\
\hline $\mathrm{C}(21)-\mathrm{C}(24)-\mathrm{H}(24 \mathrm{~A})$ & 109.5 \\
\hline $\mathrm{C}(22)-\mathrm{C}(24)-\mathrm{H}(24 \mathrm{~A})$ & 109.5 \\
\hline $\mathrm{C}(21)-\mathrm{C}(24)-\mathrm{H}(24 \mathrm{~B})$ & 109.6 \\
\hline $\mathrm{C}(22)-\mathrm{C}(24)-\mathrm{H}(24 \mathrm{~B})$ & 109.6 \\
\hline $\mathrm{H}(24 \mathrm{~A})-\mathrm{C}(24)-\mathrm{H}(24 \mathrm{~B})$ & 108.1 \\
\hline C(2)-C(1)-C(103) & $103.4(6)$ \\
\hline
\end{tabular}




\begin{tabular}{|c|c|}
\hline $\mathrm{C}(2)-\mathrm{C}(1)-\mathrm{C}(13)$ & $113.4(4)$ \\
\hline$C(103)-C(1)-C(13)$ & $142.9(11)$ \\
\hline$C(2)-C(1)-S(1)$ & $116.9(3)$ \\
\hline$C(103)-C(1)-S(1)$ & $14.1(14)$ \\
\hline $\mathrm{C}(13)-\mathrm{C}(1)-\mathrm{S}(1)$ & $129.7(4)$ \\
\hline$C(6)-C(5)-C(4)$ & $128.7(5)$ \\
\hline$C(6)-C(5)-S(2)$ & $110.6(4)$ \\
\hline$C(4)-C(5)-S(2)$ & $120.4(4)$ \\
\hline$C(19)-C(21)-C(24)$ & $110.2(5)$ \\
\hline $\mathrm{C}(19)-\mathrm{C}(21)-\mathrm{H}(21 \mathrm{~A})$ & 109.6 \\
\hline $\mathrm{C}(24)-\mathrm{C}(21)-\mathrm{H}(21 \mathrm{~A})$ & 109.6 \\
\hline $\mathrm{C}(19)-\mathrm{C}(21)-\mathrm{H}(21 \mathrm{~B})$ & 109.6 \\
\hline $\mathrm{C}(24)-\mathrm{C}(21)-\mathrm{H}(21 \mathrm{~B})$ & 109.6 \\
\hline $\mathrm{H}(21 \mathrm{~A})-\mathrm{C}(21)-\mathrm{H}(21 \mathrm{~B})$ & 108.1 \\
\hline$C(3)-C(4)-C(5)$ & $128.1(5)$ \\
\hline$C(3)-C(4)-C(103)$ & $98.4(10)$ \\
\hline$C(5)-C(4)-C(103)$ & $132.0(16)$ \\
\hline $\mathrm{C}(3)-\mathrm{C}(4)-\mathrm{S}(11)$ & $12.1(7)$ \\
\hline $\mathrm{C}(5)-\mathrm{C}(4)-\mathrm{S}(11)$ & $116.1(6)$ \\
\hline $\mathrm{C}(103)-\mathrm{C}(4)-\mathrm{S}(11)$ & $109.8(6)$ \\
\hline $\mathrm{C}(3)-\mathrm{C}(4)-\mathrm{S}(1)$ & $109.7(5)$ \\
\hline$C(5)-C(4)-S(1)$ & $121.6(4)$ \\
\hline $\mathrm{C}(103)-\mathrm{C}(4)-\mathrm{S}(1)$ & $12.0(16)$ \\
\hline $\mathrm{S}(11)-\mathrm{C}(4)-\mathrm{S}(1)$ & $121.4(5)$ \\
\hline $\mathrm{F}(2)-\mathrm{Sb}(1)-\mathrm{F}(4)$ & $93.3(3)$ \\
\hline $\mathrm{F}(2)-\mathrm{Sb}(1)-\mathrm{F}(1)$ & $91.2(3)$ \\
\hline $\mathrm{F}(4)-\mathrm{Sb}(1)-\mathrm{F}(1)$ & $175.5(3)$ \\
\hline $\mathrm{F}(2)-\mathrm{Sb}(1)-\mathrm{F}(6)$ & $91.9(2)$ \\
\hline $\mathrm{F}(4)-\mathrm{Sb}(1)-\mathrm{F}(6)$ & $90.31(17)$ \\
\hline $\mathrm{F}(1)-\mathrm{Sb}(1)-\mathrm{F}(6)$ & $88.97(18)$ \\
\hline $\mathrm{F}(2)-\mathrm{Sb}(1)-\mathrm{F}(5)$ & $90.56(18)$ \\
\hline $\mathrm{F}(4)-\mathrm{Sb}(1)-\mathrm{F}(5)$ & $90.14(17)$ \\
\hline $\mathrm{F}(1)-\mathrm{Sb}(1)-\mathrm{F}(5)$ & $90.39(18)$ \\
\hline $\mathrm{F}(6)-\mathrm{Sb}(1)-\mathrm{F}(5)$ & $177.51(18)$ \\
\hline $\mathrm{F}(2)-\mathrm{Sb}(1)-\mathrm{F}(3)$ & $178.0(2)$ \\
\hline $\mathrm{F}(4)-\mathrm{Sb}(1)-\mathrm{F}(3)$ & $88.1(3)$ \\
\hline
\end{tabular}




$\begin{array}{lc}\mathrm{F}(1)-\mathrm{Sb}(1)-\mathrm{F}(3) & 87.4(3) \\ \mathrm{F}(6)-\mathrm{Sb}(1)-\mathrm{F}(3) & 89.50(19) \\ \mathrm{F}(5)-\mathrm{Sb}(1)-\mathrm{F}(3) & 88.07(18) \\ \mathrm{C}(8)-\mathrm{S}(2)-\mathrm{C}(5) & 92.1(3) \\ \mathrm{C}(9)-\mathrm{C}(10)-\mathrm{C}(11) & 120.7(8) \\ \mathrm{C}(9)-\mathrm{C}(10)-\mathrm{H}(10) & 119.6 \\ \mathrm{C}(11)-\mathrm{C}(10)-\mathrm{H}(10) & 119.6 \\ \mathrm{C}(12)-\mathrm{S}(3)-\mathrm{C}(9) & 87.1(3) \\ \mathrm{C}(11)-\mathrm{S}(13)-\mathrm{C}(9) & 85.8(3) \\ \mathrm{C}(9)-\mathrm{C}(110)-\mathrm{C}(12) & 120.2(9) \\ \mathrm{C}(9)-\mathrm{C}(110)-\mathrm{H}(110) & 119.9 \\ \mathrm{C}(12)-\mathrm{C}(110)-\mathrm{H}(110) & 119.9 \\ \mathrm{C}(1)-\mathrm{S}(1)-\mathrm{C}(4) & 90.1(3) \\ \mathrm{C}(2)-\mathrm{C}(3)-\mathrm{C}(4) & 115.0(8) \\ \mathrm{C}(2)-\mathrm{C}(3)-\mathrm{H}(3) & 122.5 \\ \mathrm{C}(4)-\mathrm{C}(3)-\mathrm{H}(3) & 122.5 \\ \mathrm{C}(4)-\mathrm{C}(103)-\mathrm{C}(1) & 114.7(16) \\ \mathrm{C}(4)-\mathrm{C}(103)-\mathrm{H}(103) & 122.0 \\ \mathrm{C}(1)-\mathrm{C}(103)-\mathrm{H}(103) & 122.0 \\ \mathrm{C}(2)-\mathrm{S}(11)-\mathrm{C}(4) & 89.2(5)\end{array}$

Symmetry transformations used to generate equivalent atoms: 
Table 4. Anisotropic displacement parameters $\left(\AA^{2} \times 10^{3}\right)$ for $\mathbf{2}^{\cdot+} \mathbf{S b F}_{6}{ }^{-}$. The anisotropic displacement factor exponent takes the form: $\quad-2 \pi^{2}\left[\mathrm{~h}^{2} \mathrm{a}^{* 2} \mathrm{U}^{11}+\ldots \quad+2 \mathrm{~h} \mathrm{k} \mathrm{a}^{*} \mathrm{~b}^{*} \mathrm{U}^{12}\right]$

\begin{tabular}{|c|c|c|c|c|c|c|}
\hline & $\mathrm{U}^{11}$ & $\mathrm{U}^{22}$ & $\mathrm{U}^{33}$ & $\mathrm{U}^{23}$ & $\mathrm{U}^{13}$ & $\mathrm{U}^{12}$ \\
\hline C(18) & 24(3) & 44(4) & $15(3)$ & $-2(3)$ & $-2(2)$ & 4(3) \\
\hline $\mathrm{C}(12)$ & $17(3)$ & $41(4)$ & $22(3)$ & $7(3)$ & 1(2) & $2(3)$ \\
\hline $\mathrm{C}(17)$ & $21(3)$ & $31(3)$ & $30(3)$ & 2(3) & $-4(3)$ & $-1(2)$ \\
\hline $\mathrm{C}(13)$ & 29(3) & 24(3) & 21(3) & $4(2)$ & $0(3)$ & $-2(2)$ \\
\hline $\mathrm{C}(11)$ & $20(3)$ & $36(3)$ & $18(3)$ & $-3(3)$ & $4(2)$ & $-5(2)$ \\
\hline $\mathrm{C}(15)$ & $25(3)$ & $43(4)$ & $19(3)$ & 11(3) & $-1(3)$ & $-2(3)$ \\
\hline$C(16)$ & $26(3)$ & 27(3) & 20(3) & $-1(2)$ & $-6(3)$ & $6(2)$ \\
\hline $\mathrm{C}(20)$ & 21(3) & 49(4) & $22(3)$ & 7(3) & $-1(3)$ & $5(3)$ \\
\hline$C(22)$ & $22(3)$ & $25(3)$ & $39(4)$ & 2(3) & $-8(3)$ & $-3(2)$ \\
\hline$C(7)$ & $18(3)$ & 24(3) & 19(3) & $-2(2)$ & $0(2)$ & $-1(2)$ \\
\hline $\mathrm{C}(9)$ & $17(3)$ & 27(3) & 17(3) & $0(2)$ & 2(2) & $0(2)$ \\
\hline$C(2)$ & 19(3) & 20(3) & 21(3) & 2(2) & $1(2)$ & $-1(2)$ \\
\hline$C(23)$ & $28(3)$ & $50(4)$ & $21(3)$ & $-8(3)$ & $-2(3)$ & 2(3) \\
\hline$C(6)$ & $17(3)$ & $22(3)$ & $15(3)$ & $-2(2)$ & $3(2)$ & $-1(2)$ \\
\hline C(19) & $25(3)$ & $28(3)$ & 27(3) & $1(2)$ & $0(2)$ & $2(2)$ \\
\hline $\mathrm{C}(8)$ & $10(3)$ & $25(3)$ & 22(3) & $3(2)$ & $1(2)$ & $1(2)$ \\
\hline$C(14)$ & $27(4)$ & 27(3) & 18(3) & $8(2)$ & $-3(2)$ & $0(2)$ \\
\hline C(24) & $20(3)$ & $30(3)$ & $30(3)$ & 10(3) & $-4(3)$ & $-8(2)$ \\
\hline $\mathrm{C}(1)$ & $18(3)$ & $29(3)$ & 19(3) & $2(2)$ & $-1(2)$ & $2(2)$ \\
\hline$C(5)$ & $17(3)$ & $26(3)$ & $16(3)$ & $-3(2)$ & $4(2)$ & $-4(2)$ \\
\hline $\mathrm{C}(21)$ & $23(3)$ & $45(4)$ & $16(3)$ & $0(3)$ & $-1(2)$ & $6(3)$ \\
\hline $\mathrm{C}(4)$ & $22(3)$ & 23(3) & 16(3) & $4(2)$ & 2(2) & $-1(2)$ \\
\hline $\mathrm{Sb}(1)$ & $30(1)$ & $21(1)$ & $38(1)$ & $1(1)$ & $-15(1)$ & $-3(1)$ \\
\hline $\mathrm{S}(2)$ & $25(1)$ & $19(1)$ & 19(1) & $3(1)$ & $-6(1)$ & $-5(1)$ \\
\hline $\mathrm{F}(6)$ & $55(3)$ & $30(2)$ & $69(3)$ & $-16(2)$ & $-26(2)$ & $2(2)$ \\
\hline $\mathrm{F}(5)$ & $49(2)$ & $36(2)$ & $48(2)$ & $-9(2)$ & $-11(2)$ & $-13(2)$ \\
\hline $\mathrm{F}(1)$ & 49(3) & $77(3)$ & $127(5)$ & $-38(3)$ & $-50(3)$ & $26(2)$ \\
\hline $\mathrm{F}(4)$ & $54(3)$ & $56(3)$ & $127(4)$ & $-32(3)$ & $-50(3)$ & $23(2)$ \\
\hline $\mathrm{F}(2)$ & $143(5)$ & $57(3)$ & $57(3)$ & $6(2)$ & 21(3) & $-47(3)$ \\
\hline $\mathrm{F}(3)$ & $136(5)$ & 74(3) & $50(3)$ & $-8(2)$ & $17(3)$ & $-45(3)$ \\
\hline $\mathrm{C}(10)$ & $42(7)$ & $26(7)$ & $34(6)$ & $4(6)$ & $7(5)$ & $-6(6)$ \\
\hline
\end{tabular}




\begin{tabular}{lcccccc}
$\mathrm{S}(3)$ & $21(1)$ & $13(2)$ & $19(1)$ & $2(1)$ & $-6(1)$ & $1(1)$ \\
$\mathrm{S}(13)$ & $13(3)$ & $43(5)$ & $23(3)$ & $3(3)$ & $-5(2)$ & $-3(3)$ \\
$\mathrm{C}(110)$ & $0(11)$ & $42(18)$ & $37(14)$ & $41(15)$ & $6(8)$ & $2(12)$ \\
$\mathrm{S}(1)$ & $27(1)$ & $17(1)$ & $20(1)$ & $2(1)$ & $-7(1)$ & $-3(1)$ \\
$\mathrm{C}(3)$ & $22(5)$ & $20(5)$ & $26(5)$ & $-1(4)$ & $9(3)$ & $4(6)$ \\
$\mathrm{S}(11)$ & $21(11)$ & $35(15)$ & $20(11)$ & $7(10)$ & $2(8)$ & $4(13)$ \\
\hline
\end{tabular}


Table 5. Hydrogen coordinates ( $\left.\mathrm{x} 10^{4}\right)$ and isotropic displacement parameters $\left(\AA^{2} \mathrm{x} 10^{3}\right)$ for $\mathbf{2}^{\cdot+} \mathrm{SbF}_{6}^{-}$.

\begin{tabular}{|c|c|c|c|c|}
\hline & $\mathrm{x}$ & $\mathrm{y}$ & $\mathrm{z}$ & $\mathrm{U}(\mathrm{eq})$ \\
\hline $\mathrm{H}(18 \mathrm{~A})$ & 10480 & 6484 & 6857 & 33 \\
\hline $\mathrm{H}(18 \mathrm{~B})$ & 11522 & 6298 & 7359 & 33 \\
\hline $\mathrm{H}(17 \mathrm{~A})$ & 13510 & 6202 & 6888 & 33 \\
\hline $\mathrm{H}(17 \mathrm{~B})$ & 13765 & 6328 & 6078 & 33 \\
\hline $\mathrm{H}(13)$ & 11548 & 4912 & 5954 & 30 \\
\hline $\mathrm{H}(15 \mathrm{~A})$ & 11204 & 5301 & 7136 & 34 \\
\hline $\mathrm{H}(15 \mathrm{~B})$ & 10163 & 5487 & 6631 & 34 \\
\hline $\mathrm{H}(16)$ & 12189 & 6981 & 6432 & 29 \\
\hline $\mathrm{H}(20 \mathrm{~A})$ & 5126 & 6913 & 89 & 36 \\
\hline $\mathrm{H}(20 \mathrm{~B})$ & 6483 & 6998 & 170 & 36 \\
\hline $\mathrm{H}(22)$ & 5845 & 5428 & 1249 & 34 \\
\hline $\mathrm{H}(7)$ & 9165 & 7748 & 2956 & 24 \\
\hline $\mathrm{H}(23 \mathrm{~A})$ & 6711 & 5991 & 286 & 40 \\
\hline $\mathrm{H}(23 \mathrm{~B})$ & 5371 & 5903 & 138 & 40 \\
\hline $\mathrm{H}(6)$ & 10430 & 7625 & 3944 & 22 \\
\hline $\mathrm{H}(19)$ & 5592 & 7565 & 1098 & 32 \\
\hline $\mathrm{H}(14 \mathrm{~A})$ & 13458 & 5335 & 5843 & 29 \\
\hline $\mathrm{H}(14 \mathrm{~B})$ & 13218 & 5207 & 6655 & 29 \\
\hline $\mathrm{H}(24 \mathrm{~A})$ & 3958 & 5936 & 1155 & 32 \\
\hline H(24B) & 4428 & 5978 & 1940 & 32 \\
\hline $\mathrm{H}(21 \mathrm{~A})$ & 3841 & 6960 & 1123 & 34 \\
\hline $\mathrm{H}(21 \mathrm{~B})$ & 4365 & 7004 & 1895 & 34 \\
\hline $\mathrm{H}(10)$ & 7526 & 7526 & 2076 & 41 \\
\hline $\mathrm{H}(110)$ & 7757 & 5782 & 2289 & 32 \\
\hline $\mathrm{H}(3)$ & 11330 & 7208 & 5074 & 27 \\
\hline $\mathrm{H}(103)$ & 10497 & 5474 & 4605 & 86 \\
\hline
\end{tabular}


Table 6. Torsion angles $\left[{ }^{\circ}\right]$ for $\mathbf{2}^{\mathbf{}} \mathbf{S b F}_{\mathbf{6}}{ }^{-}$.

\begin{tabular}{|c|c|}
\hline $\mathrm{C}(110)-\mathrm{C}(12)-\mathrm{C}(11)-\mathrm{C}(10)$ & $1.3(14)$ \\
\hline$C(22)-C(12)-C(11)-C(10)$ & $177.1(8)$ \\
\hline$S(3)-C(12)-C(11)-C(10)$ & $-2.3(9)$ \\
\hline $\mathrm{C}(110)-\mathrm{C}(12)-\mathrm{C}(11)-\mathrm{C}(19)$ & $-174.5(12)$ \\
\hline $\mathrm{C}(22)-\mathrm{C}(12)-\mathrm{C}(11)-\mathrm{C}(19)$ & $1.3(7)$ \\
\hline $\mathrm{S}(3)-\mathrm{C}(12)-\mathrm{C}(11)-\mathrm{C}(19)$ & $-178.1(4)$ \\
\hline $\mathrm{C}(110)-\mathrm{C}(12)-\mathrm{C}(11)-\mathrm{S}(13)$ & $3.1(13)$ \\
\hline $\mathrm{C}(22)-\mathrm{C}(12)-\mathrm{C}(11)-\mathrm{S}(13)$ & $178.9(5)$ \\
\hline $\mathrm{S}(3)-\mathrm{C}(12)-\mathrm{C}(11)-\mathrm{S}(13)$ & $-0.5(8)$ \\
\hline $\mathrm{C}(1)-\mathrm{C}(13)-\mathrm{C}(15)-\mathrm{C}(18)$ & $-55.3(6)$ \\
\hline $\mathrm{C}(14)-\mathrm{C}(13)-\mathrm{C}(15)-\mathrm{C}(18)$ & $59.6(5)$ \\
\hline$C(16)-C(18)-C(15)-C(13)$ & $0.3(6)$ \\
\hline$C(15)-C(18)-C(16)-C(2)$ & $55.5(6)$ \\
\hline$C(15)-C(18)-C(16)-C(17)$ & $-59.7(6)$ \\
\hline$C(14)-C(17)-C(16)-C(2)$ & $-54.9(6)$ \\
\hline$C(14)-C(17)-C(16)-C(18)$ & $59.8(6)$ \\
\hline$C(11)-C(12)-C(22)-C(23)$ & $57.2(6)$ \\
\hline $\mathrm{C}(110)-\mathrm{C}(12)-\mathrm{C}(22)-\mathrm{C}(23)$ & $-130(2)$ \\
\hline$S(3)-C(12)-C(22)-C(23)$ & $-123.5(5)$ \\
\hline $\mathrm{C}(11)-\mathrm{C}(12)-\mathrm{C}(22)-\mathrm{C}(24)$ & $-57.5(6)$ \\
\hline $\mathrm{C}(110)-\mathrm{C}(12)-\mathrm{C}(22)-\mathrm{C}(24)$ & $115(2)$ \\
\hline$S(3)-C(12)-C(22)-C(24)$ & $121.9(5)$ \\
\hline $\mathrm{C}(18)-\mathrm{C}(16)-\mathrm{C}(2)-\mathrm{C}(1)$ & $-57.4(6)$ \\
\hline$C(17)-C(16)-C(2)-C(1)$ & $58.0(6)$ \\
\hline$C(18)-C(16)-C(2)-C(3)$ & $120.3(13)$ \\
\hline$C(17)-C(16)-C(2)-C(3)$ & $-124.4(13)$ \\
\hline $\mathrm{C}(18)-\mathrm{C}(16)-\mathrm{C}(2)-\mathrm{S}(11)$ & $117(2)$ \\
\hline $\mathrm{C}(17)-\mathrm{C}(16)-\mathrm{C}(2)-\mathrm{S}(11)$ & $-128(2)$ \\
\hline$C(19)-C(20)-C(23)-C(22)$ & $4.6(7)$ \\
\hline $\mathrm{C}(12)-\mathrm{C}(22)-\mathrm{C}(23)-\mathrm{C}(20)$ & $-58.2(6)$ \\
\hline$C(24)-C(22)-C(23)-C(20)$ & $55.5(6)$ \\
\hline$C(8)-C(7)-C(6)-C(5)$ & $0.0(7)$ \\
\hline $\mathrm{C}(12)-\mathrm{C}(11)-\mathrm{C}(19)-\mathrm{C}(21)$ & $57.5(6)$ \\
\hline$C(10)-C(11)-C(19)-C(21)$ & $-115.9(12)$ \\
\hline
\end{tabular}




\begin{tabular}{|c|c|}
\hline$S(13)-C(11)-C(19)-C(21)$ & $-120.1(6)$ \\
\hline$C(12)-C(11)-C(19)-C(20)$ & $-58.1(6)$ \\
\hline$C(10)-C(11)-C(19)-C(20)$ & $128.5(12)$ \\
\hline$S(13)-C(11)-C(19)-C(20)$ & $124.3(6)$ \\
\hline$C(23)-C(20)-C(19)-C(11)$ & $52.5(6)$ \\
\hline$C(23)-C(20)-C(19)-C(21)$ & $-62.6(6)$ \\
\hline $\mathrm{C}(10)-\mathrm{C}(9)-\mathrm{C}(8)-\mathrm{C}(7)$ & $-8.6(12)$ \\
\hline $\mathrm{C}(110)-\mathrm{C}(9)-\mathrm{C}(8)-\mathrm{C}(7)$ & $-177.5(17)$ \\
\hline $\mathrm{S}(3)-\mathrm{C}(9)-\mathrm{C}(8)-\mathrm{C}(7)$ & $176.5(5)$ \\
\hline$S(13)-C(9)-C(8)-C(7)$ & $-5.1(8)$ \\
\hline $\mathrm{C}(10)-\mathrm{C}(9)-\mathrm{C}(8)-\mathrm{S}(2)$ & $167.5(9)$ \\
\hline $\mathrm{C}(110)-\mathrm{C}(9)-\mathrm{C}(8)-\mathrm{S}(2)$ & $-1.4(18)$ \\
\hline $\mathrm{S}(3)-\mathrm{C}(9)-\mathrm{C}(8)-\mathrm{S}(2)$ & $-7.4(6)$ \\
\hline $\mathrm{S}(13)-\mathrm{C}(9)-\mathrm{C}(8)-\mathrm{S}(2)$ & $171.0(4)$ \\
\hline $\mathrm{C}(6)-\mathrm{C}(7)-\mathrm{C}(8)-\mathrm{C}(9)$ & $175.7(5)$ \\
\hline $\mathrm{C}(6)-\mathrm{C}(7)-\mathrm{C}(8)-\mathrm{S}(2)$ & $-0.7(6)$ \\
\hline $\mathrm{C}(1)-\mathrm{C}(13)-\mathrm{C}(14)-\mathrm{C}(17)$ & $54.7(6)$ \\
\hline $\mathrm{C}(15)-\mathrm{C}(13)-\mathrm{C}(14)-\mathrm{C}(17)$ & $-59.5(6)$ \\
\hline$C(16)-C(17)-C(14)-C(13)$ & $-0.6(6)$ \\
\hline$C(12)-C(22)-C(24)-C(21)$ & $53.0(6)$ \\
\hline $\mathrm{C}(23)-\mathrm{C}(22)-\mathrm{C}(24)-\mathrm{C}(21)$ & $-59.6(6)$ \\
\hline $\mathrm{C}(3)-\mathrm{C}(2)-\mathrm{C}(1)-\mathrm{C}(103)$ & $-4(5)$ \\
\hline $\mathrm{C}(16)-\mathrm{C}(2)-\mathrm{C}(1)-\mathrm{C}(103)$ & $174(5)$ \\
\hline$S(11)-C(2)-C(1)-C(103)$ & $0(6)$ \\
\hline$C(3)-C(2)-C(1)-C(13)$ & $-179.1(9)$ \\
\hline $\mathrm{C}(16)-\mathrm{C}(2)-\mathrm{C}(1)-\mathrm{C}(13)$ & $-0.8(7)$ \\
\hline $\mathrm{S}(11)-\mathrm{C}(2)-\mathrm{C}(1)-\mathrm{C}(13)$ & $-175(2)$ \\
\hline $\mathrm{C}(3)-\mathrm{C}(2)-\mathrm{C}(1)-\mathrm{S}(1)$ & $0.0(10)$ \\
\hline$C(16)-C(2)-C(1)-S(1)$ & $178.2(4)$ \\
\hline$S(11)-C(2)-C(1)-S(1)$ & $4(2)$ \\
\hline$C(15)-C(13)-C(1)-C(2)$ & $59.0(6)$ \\
\hline $\mathrm{C}(14)-\mathrm{C}(13)-\mathrm{C}(1)-\mathrm{C}(2)$ & $-57.0(6)$ \\
\hline$C(15)-C(13)-C(1)-C(103)$ & $-113(8)$ \\
\hline $\mathrm{C}(14)-\mathrm{C}(13)-\mathrm{C}(1)-\mathrm{C}(103)$ & $131(8)$ \\
\hline $\mathrm{C}(15)-\mathrm{C}(13)-\mathrm{C}(1)-\mathrm{S}(1)$ & $-120.0(5)$ \\
\hline$C(14)-C(13)-C(1)-S(1)$ & $124.1(5)$ \\
\hline
\end{tabular}




\begin{tabular}{|c|c|}
\hline$C(7)-C(6)-C(5)-C(4)$ & $-174.0(5)$ \\
\hline$C(7)-C(6)-C(5)-S(2)$ & $0.8(6)$ \\
\hline$C(11)-C(19)-C(21)-C(24)$ & $-57.1(6)$ \\
\hline$C(20)-C(19)-C(21)-C(24)$ & $58.1(6)$ \\
\hline$C(22)-C(24)-C(21)-C(19)$ & $2.4(6)$ \\
\hline$C(6)-C(5)-C(4)-C(3)$ & $8.3(14)$ \\
\hline $\mathrm{S}(2)-\mathrm{C}(5)-\mathrm{C}(4)-\mathrm{C}(3)$ & $-166.0(11)$ \\
\hline$C(6)-C(5)-C(4)-C(103)$ & 171(7) \\
\hline$S(2)-C(5)-C(4)-C(103)$ & $-3(7)$ \\
\hline$C(6)-C(5)-C(4)-S(11)$ & $10(2)$ \\
\hline$S(2)-C(5)-C(4)-S(11)$ & $-164.5(18)$ \\
\hline$C(6)-C(5)-C(4)-S(1)$ & $179.0(4)$ \\
\hline$S(2)-C(5)-C(4)-S(1)$ & $4.8(7)$ \\
\hline $\mathrm{C}(9)-\mathrm{C}(8)-\mathrm{S}(2)-\mathrm{C}(5)$ & $-175.8(4)$ \\
\hline $\mathrm{C}(7)-\mathrm{C}(8)-\mathrm{S}(2)-\mathrm{C}(5)$ & $1.0(4)$ \\
\hline$C(6)-C(5)-S(2)-C(8)$ & $-1.0(4)$ \\
\hline$C(4)-C(5)-S(2)-C(8)$ & $174.2(5)$ \\
\hline$C(110)-C(9)-C(10)-C(11)$ & $-0.9(17)$ \\
\hline$C(8)-C(9)-C(10)-C(11)$ & $-172.9(7)$ \\
\hline$S(3)-C(9)-C(10)-C(11)$ & $2.5(14)$ \\
\hline$S(13)-C(9)-C(10)-C(11)$ & $175(4)$ \\
\hline $\mathrm{C}(12)-\mathrm{C}(11)-\mathrm{C}(10)-\mathrm{C}(9)$ & $-0.3(14)$ \\
\hline $\mathrm{C}(19)-\mathrm{C}(11)-\mathrm{C}(10)-\mathrm{C}(9)$ & $173.6(7)$ \\
\hline$S(13)-C(11)-C(10)-C(9)$ & $-176(3)$ \\
\hline $\mathrm{C}(11)-\mathrm{C}(12)-\mathrm{S}(3)-\mathrm{C}(9)$ & $3.1(5)$ \\
\hline$C(110)-C(12)-S(3)-C(9)$ & $-8(4)$ \\
\hline $\mathrm{C}(22)-\mathrm{C}(12)-\mathrm{S}(3)-\mathrm{C}(9)$ & $-176.2(5)$ \\
\hline $\mathrm{C}(10)-\mathrm{C}(9)-\mathrm{S}(3)-\mathrm{C}(12)$ & $-2.9(8)$ \\
\hline $\mathrm{C}(110)-\mathrm{C}(9)-\mathrm{S}(3)-\mathrm{C}(12)$ & $11(5)$ \\
\hline $\mathrm{C}(8)-\mathrm{C}(9)-\mathrm{S}(3)-\mathrm{C}(12)$ & $173.1(5)$ \\
\hline$S(13)-C(9)-S(3)-C(12)$ & $-5.2(5)$ \\
\hline $\mathrm{C}(12)-\mathrm{C}(11)-\mathrm{S}(13)-\mathrm{C}(9)$ & $-2.5(7)$ \\
\hline $\mathrm{C}(10)-\mathrm{C}(11)-\mathrm{S}(13)-\mathrm{C}(9)$ & $3(2)$ \\
\hline $\mathrm{C}(19)-\mathrm{C}(11)-\mathrm{S}(13)-\mathrm{C}(9)$ & $175.0(5)$ \\
\hline $\mathrm{C}(10)-\mathrm{C}(9)-\mathrm{S}(13)-\mathrm{C}(11)$ & $-4(3)$ \\
\hline $\mathrm{C}(110)-\mathrm{C}(9)-\mathrm{S}(13)-\mathrm{C}(11)$ & $0.8(14)$ \\
\hline
\end{tabular}




$\begin{array}{lc}\mathrm{C}(8)-\mathrm{C}(9)-\mathrm{S}(13)-\mathrm{C}(11) & -173.4(5) \\ \mathrm{S}(3)-\mathrm{C}(9)-\mathrm{S}(13)-\mathrm{C}(11) & 5.0(6) \\ \mathrm{C}(10)-\mathrm{C}(9)-\mathrm{C}(110)-\mathrm{C}(12) & 2(2) \\ \mathrm{C}(8)-\mathrm{C}(9)-\mathrm{C}(110)-\mathrm{C}(12) & 173.7(10) \\ \mathrm{S}(3)-\mathrm{C}(9)-\mathrm{C}(110)-\mathrm{C}(12) & -165(7) \\ \mathrm{S}(13)-\mathrm{C}(9)-\mathrm{C}(110)-\mathrm{C}(12) & 1(2) \\ \mathrm{C}(11)-\mathrm{C}(12)-\mathrm{C}(110)-\mathrm{C}(9) & -2(2) \\ \mathrm{C}(22)-\mathrm{C}(12)-\mathrm{C}(110)-\mathrm{C}(9) & -175.5(8) \\ \mathrm{S}(3)-\mathrm{C}(12)-\mathrm{C}(110)-\mathrm{C}(9) & 168(6) \\ \mathrm{C}(2)-\mathrm{C}(1)-\mathrm{S}(1)-\mathrm{C}(4) & -1.2(5) \\ \mathrm{C}(103)-\mathrm{C}(1)-\mathrm{S}(1)-\mathrm{C}(4) & 16(20) \\ \mathrm{C}(13)-\mathrm{C}(1)-\mathrm{S}(1)-\mathrm{C}(4) & 177.7(5) \\ \mathrm{C}(3)-\mathrm{C}(4)-\mathrm{S}(1)-\mathrm{C}(1) & 2.0(9) \\ \mathrm{C}(5)-\mathrm{C}(4)-\mathrm{S}(1)-\mathrm{C}(1) & -170.3(5) \\ \mathrm{C}(103)-\mathrm{C}(4)-\mathrm{S}(1)-\mathrm{C}(1) & -19(23) \\ \mathrm{S}(11)-\mathrm{C}(4)-\mathrm{S}(1)-\mathrm{C}(1) & -2(2) \\ \mathrm{C}(1)-\mathrm{C}(2)-\mathrm{C}(3)-\mathrm{C}(4) & 1.7(16) \\ \mathrm{C}(16)-\mathrm{C}(2)-\mathrm{C}(3)-\mathrm{C}(4) & -176.1(7) \\ \mathrm{S}(11)-\mathrm{C}(2)-\mathrm{C}(3)-\mathrm{C}(4) & -165(11) \\ \mathrm{C}(5)-\mathrm{C}(4)-\mathrm{C}(3)-\mathrm{C}(2) & 169.1(8) \\ \mathrm{C}(103)-\mathrm{C}(4)-\mathrm{C}(3)-\mathrm{C}(2) & 2(5) \\ \mathrm{S}(11)-\mathrm{C}(4)-\mathrm{C}(3)-\mathrm{C}(2) & 163(12) \\ \mathrm{S}(1)-\mathrm{C}(4)-\mathrm{C}(3)-\mathrm{C}(2) & -13(9) \\ \mathrm{C}(3)-\mathrm{C}(4)-\mathrm{C}(103)-\mathrm{C}(1) & -2.5(16) \\ \mathrm{C}(5)-\mathrm{C}(4)-\mathrm{C}(103)-\mathrm{C}(1) & -5(9) \\ \mathrm{S}(11)-\mathrm{C}(4)-\mathrm{C}(103)-\mathrm{C}(1) & -171(3) \\ \mathrm{S}(1)-\mathrm{C}(4)-\mathrm{C}(103)-\mathrm{C}(1) & -9(10) \\ \mathrm{C}(2)-\mathrm{C}(1)-\mathrm{C}(103)-\mathrm{C}(4) & 155(31) \\ \mathrm{C}(13)-\mathrm{C}(1)-\mathrm{C}(103)-\mathrm{C}(4) & 6(9) \\ \mathrm{S}(1)-\mathrm{C}(1)-\mathrm{C}(103)-\mathrm{C}(4) & -4(3) \\ \mathrm{C}(1)-\mathrm{C}(2)-\mathrm{S}(11)-\mathrm{C}(4) & 11(8) \\ \mathrm{C}(3)-\mathrm{C}(2)-\mathrm{S}(11)-\mathrm{C}(4) & \\ \mathrm{C}(16)-\mathrm{C}(2)-\mathrm{S}(11)-\mathrm{C}(4) & \\ \mathrm{C}(3)-\mathrm{C}(4)-\mathrm{S}(11)-\mathrm{C}(2) & \\ \mathrm{C}(5)-\mathrm{C}(4)-\mathrm{S}(11)-\mathrm{C}(2) & (14) \\ \mathrm{C}(103)-\mathrm{C}(4)-\mathrm{S}(11)-\mathrm{C}(2) & \\ & \\ & \end{array}$


$\mathrm{S}(1)-\mathrm{C}(4)-\mathrm{S}(11)-\mathrm{C}(2)$

$3(3)$

$\mathrm{C}(3)-\mathrm{C}(4)-\mathrm{C}(5)-\mathrm{C}(6)$

$8.3(14)$

$\mathrm{C}(7)-\mathrm{C}(8)-\mathrm{C}(9)-\mathrm{C}(10)$

$-8.6(12)$

$\mathrm{S}(1)-\mathrm{C}(4)-\mathrm{C}(5)-\mathrm{S}(2)$

$4.8(7)$

$\mathrm{S}(2)-\mathrm{C}(8)-\mathrm{C}(9)-\mathrm{S}(3)$

$-7.4(6)$

Symmetry transformations used to generate equivalent atoms: 
X-Ray Crystallography of $2^{\circ+} \mathrm{SbF}_{6}^{-}$(298K).
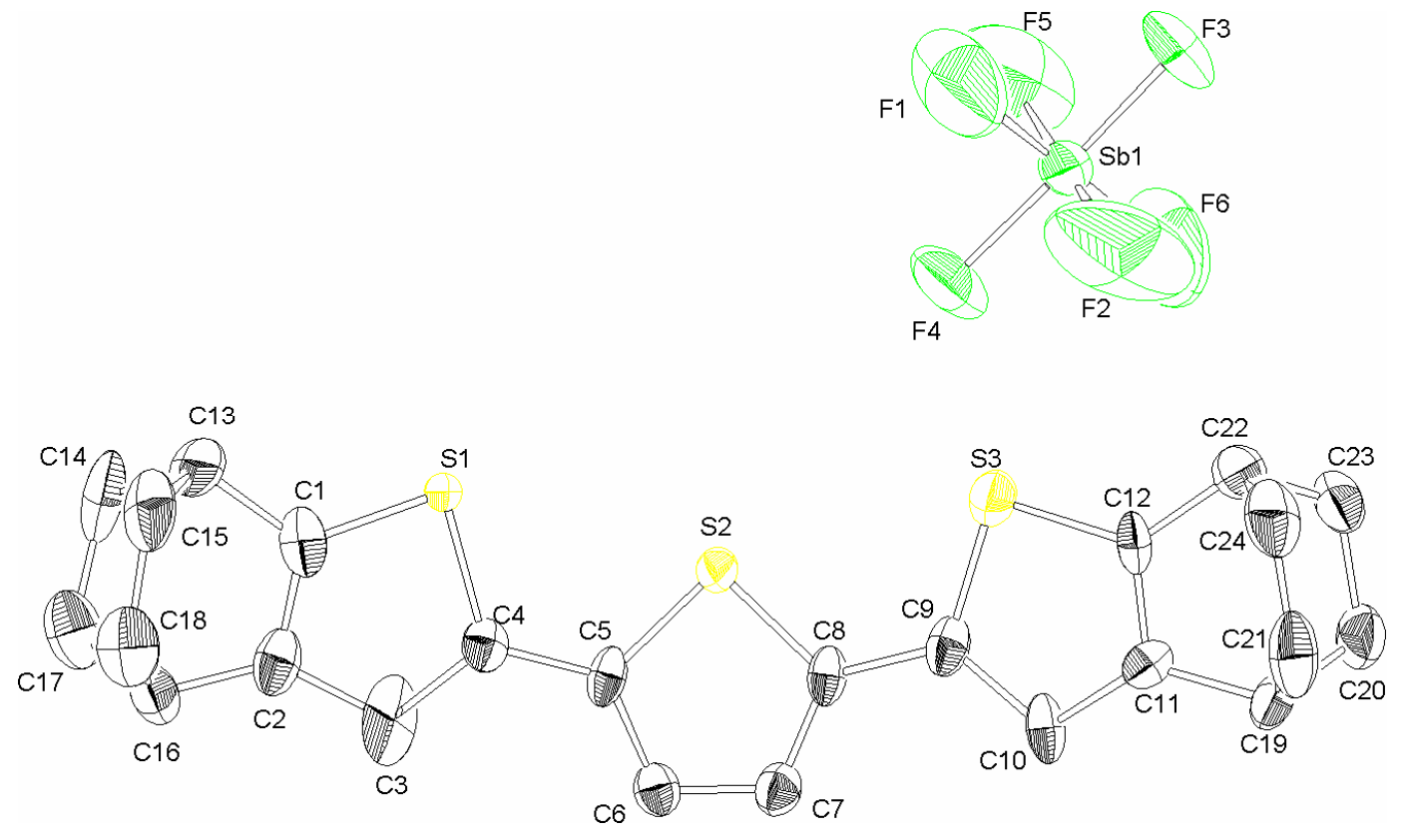
Table 1. Crystal data and structure refinement for $\mathbf{2}^{\cdot+} \mathbf{S b F}_{6}^{-}$.

Identification code

Empirical formula

Formula weight

Temperature

Wavelength

Crystal system

Space group

Unit cell dimensions

Volume

Z

Density (calculated)

Absorption coefficient

$\mathrm{F}(000)$

Crystal size

Theta range for data collection

Index ranges

Reflections collected

Independent reflections

Completeness to theta $=25.00^{\circ}$

Absorption correction

Max. and min. transmission

Refinement method

Data / restraints / parameters

Goodness-of-fit on $\mathrm{F}^{2}$

Final $\mathrm{R}$ indices $[\mathrm{I}>2 \operatorname{sigma}(\mathrm{I})]$

$\mathrm{R}$ indices (all data)

Largest diff. peak and hole
$\mathrm{RTpD}$

C24 H24 F6 S3 Sb

644.36

298(2) K

$0.71073 \AA$

Orthorhombic

Pbcn

$\begin{array}{ll}\mathrm{a}=11.7896(13) \AA & \alpha=90^{\circ} . \\ \mathrm{b}=21.747(2) \AA & \beta=90^{\circ} . \\ \mathrm{c}=19.296(2) \AA & \gamma=90^{\circ} .\end{array}$

4947.1(10) $\AA^{3}$

8

$1.730 \mathrm{Mg} / \mathrm{m}^{3}$

$1.426 \mathrm{~mm}^{-1}$

2568

$0.15 \times 0.15 \times 0.10 \mathrm{~mm}^{3}$

1.87 to $25.00^{\circ}$.

$-12<=\mathrm{h}<=14,-25<=\mathrm{k}<=23,-22<=\mathrm{l}<=22$

23652

$4357[\mathrm{R}(\mathrm{int})=0.0695]$

$100.0 \%$

Empirical

0.8706 and 0.8146

Full-matrix least-squares on $\mathrm{F}^{2}$

4357 / 6 / 326

1.412

$\mathrm{R} 1=0.1277, \mathrm{wR} 2=0.2476$

$\mathrm{R} 1=0.1468, \mathrm{wR} 2=0.2559$

1.790 and -0.496 e. $\AA^{-3}$ 
Table 2. Atomic coordinates (x 10 $)$ and equivalent isotropic displacement parameters $\left(\AA^{2} \times 10^{3}\right)$ for $\mathbf{2}^{+} \mathbf{S b F}_{\mathbf{6}}{ }^{-}$. $\quad \mathrm{U}(\mathrm{eq})$ is defined as one third of the trace of the orthogonalized $\mathrm{U}^{\mathrm{ij}}$ tensor.

\begin{tabular}{|c|c|c|c|c|}
\hline & $\mathrm{x}$ & $\mathrm{y}$ & $\mathrm{z}$ & $\mathrm{U}(\mathrm{eq})$ \\
\hline $\mathrm{Sb}(1)$ & $2247(1)$ & $1046(1)$ & $9077(1)$ & $58(1)$ \\
\hline $\mathrm{S}(2)$ & $4376(3)$ & $1162(1)$ & $1589(2)$ & $34(1)$ \\
\hline $\mathrm{S}(3)$ & $5794(3)$ & $648(2)$ & $288(2)$ & $47(1)$ \\
\hline $\mathrm{S}(1)$ & $2653(7)$ & $902(3)$ & $2841(4)$ & $31(2)$ \\
\hline$C(3)$ & $2430(30)$ & $2098(15)$ & $2964(16)$ & $69(12)$ \\
\hline$S(101)$ & $2436(15)$ & $2338(7)$ & 2993(13) & $63(7)$ \\
\hline$C(103)$ & $2560(40)$ & $1137(17)$ & $2850(30)$ & $50(20)$ \\
\hline $\mathrm{F}(1)$ & $3519(14)$ & $1444(7)$ & $8838(12)$ & $204(10)$ \\
\hline$C(4)$ & $3054(9)$ & $1668(4)$ & $2611(6)$ & $31(3)$ \\
\hline $\mathrm{C}(11)$ & $6538(10)$ & $1415(5)$ & $-618(6)$ & $33(3)$ \\
\hline$C(5)$ & $3865(9)$ & $1769(5)$ & $2076(5)$ & $30(3)$ \\
\hline$F(3)$ & $1767(10)$ & $1716(5)$ & $9595(6)$ & $109(4)$ \\
\hline$C(12)$ & $6372(10)$ & $798(6)$ & $-509(6)$ & $38(3)$ \\
\hline$C(10)$ & $6186(9)$ & $1803(6)$ & $-81(5)$ & $34(3)$ \\
\hline $\mathrm{C}(13)$ & $787(11)$ & $887(6)$ & $3781(8)$ & $50(4)$ \\
\hline$C(7)$ & $5020(10)$ & $2262(5)$ & $1284(6)$ & $34(3)$ \\
\hline$F(4)$ & $2647(11)$ & $391(5)$ & $8496(7)$ & $124(5)$ \\
\hline$C(8)$ & $5145(9)$ & $1652(5)$ & $1068(5)$ & $29(2)$ \\
\hline$C(22)$ & $6701(12)$ & $371(6)$ & $-1072(6)$ & $44(3)$ \\
\hline$C(19)$ & $7019(11)$ & $1531(6)$ & $-1335(6)$ & $42(3)$ \\
\hline$C(6)$ & $4307(9)$ & $2326(5)$ & $1837(6)$ & $32(3)$ \\
\hline$C(2)$ & $1563(9)$ & $1853(6)$ & $3421(6)$ & $42(3)$ \\
\hline $\mathrm{C}(21)$ & $6173(13)$ & $1254(8)$ & $-1854(7)$ & $60(4)$ \\
\hline$C(16)$ & $652(11)$ & $2074(6)$ & $3865(6)$ & $44(3)$ \\
\hline$C(20)$ & $8128(11)$ & $1174(6)$ & $-1410(7)$ & $48(3)$ \\
\hline$C(17)$ & $818(12)$ & $1805(7)$ & $4586(7)$ & $58(4)$ \\
\hline$C(9)$ & $5724(10)$ & $1433(5)$ & $476(6)$ & $35(3)$ \\
\hline $\mathrm{C}(1)$ & $1662(10)$ & $1223(6)$ & $3365(6)$ & $46(3)$ \\
\hline$C(23)$ & $7934(11)$ & 493(6) & $-1244(7)$ & $46(3)$ \\
\hline$C(24)$ & $5977(13)$ & $567(7)$ & $-1692(7)$ & $57(4)$ \\
\hline $\mathrm{F}(2)$ & $2820(20)$ & $676(8)$ & $9803(9)$ & $232(12)$ \\
\hline
\end{tabular}




$\begin{array}{lrrrr}\mathrm{F}(6) & 885(13) & 664(8) & 9226(11) & 200(10) \\ \mathrm{C}(18) & -450(11) & 1818(7) & 3575(8) & 57(4) \\ \mathrm{C}(15) & -408(11) & 1107(7) & 3534(8) & 60(4) \\ \mathrm{C}(14) & 956(12) & 1108(8) & 4532(7) & 66(5) \\ \mathrm{F}(5) & 1600(20) & 1442(8) & 8303(8) & 194(9)\end{array}$


Table 3. Bond lengths $[\AA]$ and angles $\left[{ }^{\circ}\right]$ for $\mathbf{2}^{\mathbf{}} \mathbf{S b F}_{\mathbf{6}}^{-}$.

\begin{tabular}{|c|c|}
\hline $\mathrm{Sb}(1)-\mathrm{F}(2)$ & $1.749(15)$ \\
\hline $\mathrm{Sb}(1)-\mathrm{F}(1)$ & $1.792(13)$ \\
\hline $\mathrm{Sb}(1)-\mathrm{F}(6)$ & $1.830(12)$ \\
\hline $\mathrm{Sb}(1)-\mathrm{F}(3)$ & $1.856(9)$ \\
\hline $\mathrm{Sb}(1)-\mathrm{F}(4)$ & $1.871(9)$ \\
\hline $\mathrm{Sb}(1)-\mathrm{F}(5)$ & $1.888(16)$ \\
\hline $\mathrm{S}(2)-\mathrm{C}(8)$ & $1.723(11)$ \\
\hline$S(2)-C(5)$ & $1.729(11)$ \\
\hline$S(3)-C(12)$ & $1.715(12)$ \\
\hline$S(3)-C(9)$ & $1.746(12)$ \\
\hline $\mathrm{S}(1)-\mathrm{C}(1)$ & $1.696(12)$ \\
\hline $\mathrm{S}(1)-\mathrm{C}(4)$ & $1.788(10)$ \\
\hline$C(3)-C(4)$ & $1.37(3)$ \\
\hline$C(3)-C(2)$ & $1.45(3)$ \\
\hline$S(101)-C(2)$ & $1.689(14)$ \\
\hline$S(101)-C(4)$ & $1.787(12)$ \\
\hline$C(103)-C(4)$ & $1.38(3)$ \\
\hline $\mathrm{C}(103)-\mathrm{C}(1)$ & $1.46(4)$ \\
\hline$C(4)-C(5)$ & $1.424(15)$ \\
\hline $\mathrm{C}(11)-\mathrm{C}(12)$ & $1.371(16)$ \\
\hline $\mathrm{C}(11)-\mathrm{C}(10)$ & $1.399(16)$ \\
\hline $\mathrm{C}(11)-\mathrm{C}(19)$ & $1.518(16)$ \\
\hline$C(5)-C(6)$ & $1.396(15)$ \\
\hline $\mathrm{C}(12)-\mathrm{C}(22)$ & $1.481(16)$ \\
\hline$C(10)-C(9)$ & $1.449(16)$ \\
\hline$C(13)-C(1)$ & $1.498(15)$ \\
\hline $\mathrm{C}(13)-\mathrm{C}(14)$ & $1.54(2)$ \\
\hline$C(13)-C(15)$ & $1.563(19)$ \\
\hline$C(7)-C(6)$ & $1.365(15)$ \\
\hline$C(7)-C(8)$ & $1.398(15)$ \\
\hline $\mathrm{C}(8)-\mathrm{C}(9)$ & $1.414(15)$ \\
\hline $\mathrm{C}(22)-\mathrm{C}(23)$ & $1.514(18)$ \\
\hline$C(22)-C(24)$ & $1.530(19)$ \\
\hline$C(19)-C(20)$ & $1.527(18)$ \\
\hline
\end{tabular}




\begin{tabular}{|c|c|}
\hline $\mathrm{C}(19)-\mathrm{C}(21)$ & $1.536(19)$ \\
\hline $\mathrm{C}(2)-\mathrm{C}(1)$ & $1.378(19)$ \\
\hline$C(2)-C(16)$ & $1.457(15)$ \\
\hline$C(21)-C(24)$ & $1.54(2)$ \\
\hline $\mathrm{C}(16)-\mathrm{C}(18)$ & $1.520(18)$ \\
\hline$C(16)-C(17)$ & $1.521(18)$ \\
\hline $\mathrm{C}(20)-\mathrm{C}(23)$ & $1.532(18)$ \\
\hline$C(17)-C(14)$ & $1.53(2)$ \\
\hline$C(18)-C(15)$ & $1.55(2)$ \\
\hline $\mathrm{F}(2)-\mathrm{Sb}(1)-\mathrm{F}(1)$ & $96.1(12)$ \\
\hline $\mathrm{F}(2)-\mathrm{Sb}(1)-\mathrm{F}(6)$ & $90.3(12)$ \\
\hline $\mathrm{F}(1)-\mathrm{Sb}(1)-\mathrm{F}(6)$ & $173.5(11)$ \\
\hline $\mathrm{F}(2)-\mathrm{Sb}(1)-\mathrm{F}(3)$ & $92.7(7)$ \\
\hline $\mathrm{F}(1)-\mathrm{Sb}(1)-\mathrm{F}(3)$ & $90.8(6)$ \\
\hline $\mathrm{F}(6)-\mathrm{Sb}(1)-\mathrm{F}(3)$ & $90.2(6)$ \\
\hline $\mathrm{F}(2)-\mathrm{Sb}(1)-\mathrm{F}(4)$ & $91.9(7)$ \\
\hline $\mathrm{F}(1)-\mathrm{Sb}(1)-\mathrm{F}(4)$ & $90.1(6)$ \\
\hline $\mathrm{F}(6)-\mathrm{Sb}(1)-\mathrm{F}(4)$ & $88.3(6)$ \\
\hline $\mathrm{F}(3)-\mathrm{Sb}(1)-\mathrm{F}(4)$ & $175.1(6)$ \\
\hline $\mathrm{F}(2)-\mathrm{Sb}(1)-\mathrm{F}(5)$ & $178.7(12)$ \\
\hline $\mathrm{F}(1)-\mathrm{Sb}(1)-\mathrm{F}(5)$ & $85.1(11)$ \\
\hline $\mathrm{F}(6)-\mathrm{Sb}(1)-\mathrm{F}(5)$ & $88.5(11)$ \\
\hline $\mathrm{F}(3)-\mathrm{Sb}(1)-\mathrm{F}(5)$ & $86.8(6)$ \\
\hline $\mathrm{F}(4)-\mathrm{Sb}(1)-\mathrm{F}(5)$ & $88.6(7)$ \\
\hline$C(8)-S(2)-C(5)$ & $91.6(5)$ \\
\hline$C(12)-S(3)-C(9)$ & $91.1(6)$ \\
\hline$C(1)-S(1)-C(4)$ & $86.9(6)$ \\
\hline$C(4)-C(3)-C(2)$ & $115(2)$ \\
\hline $\mathrm{C}(2)-\mathrm{S}(101)-\mathrm{C}(4)$ & $86.6(7)$ \\
\hline $\mathrm{C}(4)-\mathrm{C}(103)-\mathrm{C}(1)$ & $115(2)$ \\
\hline $\mathrm{C}(3)-\mathrm{C}(4)-\mathrm{C}(103)$ & $100(3)$ \\
\hline$C(3)-C(4)-C(5)$ & $128.0(15)$ \\
\hline$C(103)-C(4)-C(5)$ & $131.3(18)$ \\
\hline$C(3)-C(4)-S(101)$ & $11.7(16)$ \\
\hline$C(103)-C(4)-S(101)$ & $111.8(15)$ \\
\hline
\end{tabular}




\begin{tabular}{|c|c|}
\hline$C(5)-C(4)-S(101)$ & $116.6(9)$ \\
\hline$C(3)-C(4)-S(1)$ & $111.7(15)$ \\
\hline $\mathrm{C}(103)-\mathrm{C}(4)-\mathrm{S}(1)$ & $11.8(17)$ \\
\hline$C(5)-C(4)-S(1)$ & $120.1(8)$ \\
\hline$S(101)-C(4)-S(1)$ & $123.3(8)$ \\
\hline$C(12)-C(11)-C(10)$ & $115.8(11)$ \\
\hline$C(12)-C(11)-C(19)$ & $110.8(10)$ \\
\hline $\mathrm{C}(10)-\mathrm{C}(11)-\mathrm{C}(19)$ & $133.3(11)$ \\
\hline$C(6)-C(5)-C(4)$ & $128.5(10)$ \\
\hline$C(6)-C(5)-S(2)$ & $110.7(8)$ \\
\hline $\mathrm{C}(4)-\mathrm{C}(5)-\mathrm{S}(2)$ & $120.7(8)$ \\
\hline $\mathrm{C}(11)-\mathrm{C}(12)-\mathrm{C}(22)$ & $117.7(11)$ \\
\hline$C(11)-C(12)-S(3)$ & $112.3(9)$ \\
\hline $\mathrm{C}(22)-\mathrm{C}(12)-\mathrm{S}(3)$ & $130.0(10)$ \\
\hline $\mathrm{C}(11)-\mathrm{C}(10)-\mathrm{C}(9)$ & $109.0(11)$ \\
\hline $\mathrm{C}(1)-\mathrm{C}(13)-\mathrm{C}(14)$ & $105.3(11)$ \\
\hline $\mathrm{C}(1)-\mathrm{C}(13)-\mathrm{C}(15)$ & $107.9(11)$ \\
\hline $\mathrm{C}(14)-\mathrm{C}(13)-\mathrm{C}(15)$ & $108.0(11)$ \\
\hline$C(6)-C(7)-C(8)$ & $113.2(10)$ \\
\hline $\mathrm{C}(7)-\mathrm{C}(8)-\mathrm{C}(9)$ & $127.8(10)$ \\
\hline $\mathrm{C}(7)-\mathrm{C}(8)-\mathrm{S}(2)$ & $110.9(8)$ \\
\hline $\mathrm{C}(9)-\mathrm{C}(8)-\mathrm{S}(2)$ & $121.0(9)$ \\
\hline$C(12)-C(22)-C(23)$ & $107.6(10)$ \\
\hline$C(12)-C(22)-C(24)$ & $104.6(11)$ \\
\hline$C(23)-C(22)-C(24)$ & $108.4(11)$ \\
\hline$C(11)-C(19)-C(20)$ & $108.8(10)$ \\
\hline $\mathrm{C}(11)-\mathrm{C}(19)-\mathrm{C}(21)$ & $106.6(10)$ \\
\hline $\mathrm{C}(20)-\mathrm{C}(19)-\mathrm{C}(21)$ & $107.2(11)$ \\
\hline$C(7)-C(6)-C(5)$ & $113.5(10)$ \\
\hline$C(1)-C(2)-C(3)$ & $105.0(14)$ \\
\hline$C(1)-C(2)-C(16)$ & $115.8(9)$ \\
\hline$C(3)-C(2)-C(16)$ & 139.1(17) \\
\hline$C(1)-C(2)-S(101)$ & $122.0(6)$ \\
\hline $\mathrm{C}(3)-\mathrm{C}(2)-\mathrm{S}(101)$ & $17.1(14)$ \\
\hline$C(16)-C(2)-S(101)$ & $122.1(10)$ \\
\hline$C(19)-C(21)-C(24)$ & $110.1(11)$ \\
\hline
\end{tabular}




$\begin{array}{lc}\mathrm{C}(2)-\mathrm{C}(16)-\mathrm{C}(18) & 107.0(10) \\ \mathrm{C}(2)-\mathrm{C}(16)-\mathrm{C}(17) & 108.5(11) \\ \mathrm{C}(18)-\mathrm{C}(16)-\mathrm{C}(17) & 107.8(12) \\ \mathrm{C}(19)-\mathrm{C}(20)-\mathrm{C}(23) & 110.1(10) \\ \mathrm{C}(16)-\mathrm{C}(17)-\mathrm{C}(14) & 109.4(11) \\ \mathrm{C}(8)-\mathrm{C}(9)-\mathrm{C}(10) & 126.3(11) \\ \mathrm{C}(8)-\mathrm{C}(9)-\mathrm{S}(3) & 121.4(9) \\ \mathrm{C}(10)-\mathrm{C}(9)-\mathrm{S}(3) & 111.8(8) \\ \mathrm{C}(2)-\mathrm{C}(1)-\mathrm{C}(103) & 104.1(14) \\ \mathrm{C}(2)-\mathrm{C}(1)-\mathrm{C}(13) & 112.6(9) \\ \mathrm{C}(103)-\mathrm{C}(1)-\mathrm{C}(13) & 143.0(16) \\ \mathrm{C}(2)-\mathrm{C}(1)-\mathrm{S}(1) & 121.0(5) \\ \mathrm{C}(103)-\mathrm{C}(1)-\mathrm{S}(1) & 17.0(15) \\ \mathrm{C}(13)-\mathrm{C}(1)-\mathrm{S}(1) & 126.4(10) \\ \mathrm{C}(22)-\mathrm{C}(23)-\mathrm{C}(20) & 111.0(11) \\ \mathrm{C}(22)-\mathrm{C}(24)-\mathrm{C}(21) & 110.2(11) \\ \mathrm{C}(16)-\mathrm{C}(18)-\mathrm{C}(15) & 111.0(11) \\ \mathrm{C}(18)-\mathrm{C}(15)-\mathrm{C}(13) & 108.5(10) \\ \mathrm{C}(17)-\mathrm{C}(14)-\mathrm{C}(13) & 111.1(12)\end{array}$

Symmetry transformations used to generate equivalent atoms: 
Table 4. Anisotropic displacement parameters $\left(\AA^{2} \times 10^{3}\right)$ for $\mathbf{2}^{\cdot+} \mathbf{S b F}_{6}{ }^{-}$. The anisotropic displacement factor exponent takes the form: $\quad-2 \pi^{2}\left[\mathrm{~h}^{2} \mathrm{a}^{* 2} \mathrm{U}^{11}+\ldots \quad+2 \mathrm{hka} \mathrm{a}^{*} \mathrm{U}^{12}\right]$

\begin{tabular}{|c|c|c|c|c|c|c|}
\hline & $\mathrm{U}^{11}$ & $\mathrm{U}^{22}$ & $\mathrm{U}^{33}$ & $\mathrm{U}^{23}$ & $\mathrm{U}^{13}$ & $\mathrm{U}^{12}$ \\
\hline $\mathrm{Sb}(1)$ & $54(1)$ & $40(1)$ & $81(1)$ & $4(1)$ & $31(1)$ & $9(1)$ \\
\hline $\mathrm{S}(2)$ & $42(2)$ & $27(1)$ & $34(2)$ & $-6(1)$ & $15(1)$ & $-9(1)$ \\
\hline $\mathrm{S}(3)$ & $62(2)$ & $42(2)$ & $38(2)$ & $-4(1)$ & $18(2)$ & $-10(2)$ \\
\hline$S(1)$ & $34(3)$ & $20(4)$ & $38(3)$ & 2(3) & $18(2)$ & 2(3) \\
\hline $\mathrm{C}(3)$ & $90(30)$ & $110(30)$ & $3(11)$ & $-8(15)$ & $-4(12)$ & $-10(20)$ \\
\hline$S(101)$ & $24(7)$ & $108(15)$ & $58(11)$ & $-20(9)$ & $10(6)$ & 2(7) \\
\hline$C(103)$ & $40(30)$ & $40(40)$ & $60(30)$ & $-50(30)$ & $0(30)$ & $-30(30)$ \\
\hline $\mathrm{F}(1)$ & $136(13)$ & $138(13)$ & $340(30)$ & $-99(15)$ & $137(16)$ & $-60(10)$ \\
\hline $\mathrm{C}(4)$ & $31(6)$ & $38(6)$ & $23(6)$ & $-5(5)$ & $-3(5)$ & $-5(5)$ \\
\hline $\mathrm{C}(11)$ & $34(7)$ & $32(6)$ & $33(6)$ & $5(5)$ & $0(5)$ & $-6(5)$ \\
\hline$C(5)$ & $20(6)$ & 49(7) & $20(5)$ & $0(5)$ & $0(4)$ & $-9(5)$ \\
\hline $\mathrm{F}(3)$ & $129(10)$ & $71(6)$ & $127(9)$ & $-32(6)$ & $52(8)$ & $34(6)$ \\
\hline$C(12)$ & $32(7)$ & $52(7)$ & $31(7)$ & $-5(6)$ & $15(5)$ & $6(6)$ \\
\hline $\mathrm{C}(10)$ & $23(6)$ & $64(8)$ & $15(5)$ & $-11(5)$ & $-4(4)$ & $2(5)$ \\
\hline$C(13)$ & $31(7)$ & $47(8)$ & $73(10)$ & $6(7)$ & $18(7)$ & $-8(6)$ \\
\hline$C(7)$ & $37(7)$ & $38(6)$ & $27(6)$ & $0(5)$ & $3(5)$ & $-4(5)$ \\
\hline $\mathrm{F}(4)$ & $139(10)$ & $60(6)$ & $173(12)$ & $-50(7)$ & $75(9)$ & $-5(6)$ \\
\hline$C(8)$ & $27(6)$ & $48(7)$ & $14(5)$ & $-1(5)$ & $-1(4)$ & $-2(5)$ \\
\hline $\mathrm{C}(22)$ & $62(9)$ & $38(7)$ & $33(7)$ & $-6(5)$ & $5(6)$ & $-6(6)$ \\
\hline $\mathrm{C}(19)$ & $43(8)$ & $46(7)$ & $36(7)$ & $10(6)$ & $14(6)$ & $-1(6)$ \\
\hline$C(6)$ & $30(6)$ & $37(6)$ & $28(6)$ & $-5(5)$ & $-1(5)$ & $0(5)$ \\
\hline$C(2)$ & $40(7)$ & $62(9)$ & $23(6)$ & $0(6)$ & $0(5)$ & $-9(6)$ \\
\hline $\mathrm{C}(21)$ & $53(9)$ & $99(12)$ & $27(7)$ & $-6(7)$ & $3(6)$ & $9(8)$ \\
\hline$C(16)$ & $48(8)$ & $47(7)$ & $38(7)$ & $-8(6)$ & $7(6)$ & $7(6)$ \\
\hline$C(20)$ & $38(7)$ & $55(8)$ & $52(8)$ & $-6(7)$ & $15(6)$ & $-7(6)$ \\
\hline $\mathrm{C}(17)$ & $39(8)$ & $80(11)$ & $53(9)$ & $-11(8)$ & $-2(7)$ & 13(7) \\
\hline $\mathrm{C}(9)$ & $33(7)$ & $50(7)$ & $23(6)$ & $-1(5)$ & $2(5)$ & $-9(5)$ \\
\hline $\mathrm{C}(1)$ & $41(8)$ & 64(9) & $34(7)$ & $-8(6)$ & $6(6)$ & $3(6)$ \\
\hline$C(23)$ & $44(8)$ & $49(8)$ & $44(7)$ & $-14(6)$ & $7(6)$ & $2(6)$ \\
\hline$C(24)$ & $60(9)$ & $77(10)$ & $33(7)$ & $-22(7)$ & $-1(7)$ & $-6(8)$ \\
\hline $\mathrm{F}(2)$ & $410(30)$ & $132(13)$ & 151(15) & $23(11)$ & $-72(18)$ & $128(17)$ \\
\hline
\end{tabular}




$\begin{array}{lcccccc}\mathrm{F}(6) & 118(12) & 174(14) & 310(20) & -98(15) & 129(14) & -74(11) \\ \mathrm{C}(18) & 34(8) & 84(11) & 54(9) & -3(8) & -7(6) & 5(7) \\ \mathrm{C}(15) & 32(7) & 88(11) & 60(9) & -21(8) & 14(6) & -27(7) \\ \mathrm{C}(14) & 41(8) & 120(14) & 35(8) & 19(9) & 15(6) & 6(9) \\ \mathrm{F}(5) & 310(30) & 152(14) & 120(12) & -13(10) & -40(15) & 63(16)\end{array}$


Table 5. Hydrogen coordinates ( $\left.\mathrm{x} 10^{4}\right)$ and isotropic displacement parameters $\left(\AA^{2} \mathrm{x} 10^{3}\right)$ for $2^{++} \mathrm{SbF}_{6}^{-}$.

\begin{tabular}{|c|c|c|c|c|}
\hline & $\mathrm{x}$ & $\mathrm{y}$ & $\mathrm{z}$ & $\mathrm{U}(\mathrm{eq})$ \\
\hline $\mathrm{H}(3)$ & 2552 & 2519 & 2914 & 83 \\
\hline $\mathrm{H}(103)$ & 2782 & 749 & 2703 & 55 \\
\hline $\mathrm{H}(10)$ & 6241 & 2230 & -81 & 41 \\
\hline $\mathrm{H}(13)$ & 872 & 440 & 3741 & 60 \\
\hline $\mathrm{H}(7)$ & 5385 & 2592 & 1073 & 41 \\
\hline $\mathrm{H}(22)$ & 6570 & -59 & -945 & 53 \\
\hline $\mathrm{H}(19)$ & 7133 & 1971 & -1419 & 50 \\
\hline $\mathrm{H}(6)$ & 4132 & 2704 & 2036 & 38 \\
\hline $\mathrm{H}(21 \mathrm{~A})$ & 6466 & 1298 & -2321 & 71 \\
\hline $\mathrm{H}(21 \mathrm{~B})$ & 5459 & 1474 & -1827 & 71 \\
\hline $\mathrm{H}(16)$ & 636 & 2524 & 3880 & 53 \\
\hline $\mathrm{H}(20 \mathrm{~A})$ & 8692 & 1342 & -1097 & 58 \\
\hline $\mathrm{H}(20 \mathrm{~B})$ & 8411 & 1215 & -1880 & 58 \\
\hline $\mathrm{H}(17 \mathrm{~A})$ & 168 & 1901 & 4874 & 69 \\
\hline $\mathrm{H}(17 \mathrm{~B})$ & 1487 & 1983 & 4799 & 69 \\
\hline $\mathrm{H}(23 \mathrm{~A})$ & 8155 & 245 & -1640 & 55 \\
\hline $\mathrm{H}(23 \mathrm{~B})$ & 8405 & 375 & -854 & 55 \\
\hline $\mathrm{H}(24 \mathrm{~A})$ & 5182 & 498 & -1589 & 68 \\
\hline $\mathrm{H}(24 \mathrm{~B})$ & 6176 & 321 & -2093 & 68 \\
\hline $\mathrm{H}(18 \mathrm{~A})$ & -579 & 1985 & 3116 & 69 \\
\hline $\mathrm{H}(18 \mathrm{~B})$ & -1076 & 1942 & 3870 & 69 \\
\hline $\mathrm{H}(15 \mathrm{~A})$ & -541 & 973 & 3061 & 72 \\
\hline $\mathrm{H}(15 \mathrm{~B})$ & -993 & 930 & 3827 & 72 \\
\hline $\mathrm{H}(14 \mathrm{~A})$ & 406 & 908 & 4830 & 79 \\
\hline $\mathrm{H}(14 \mathrm{~B})$ & 1708 & 993 & 4689 & 79 \\
\hline
\end{tabular}


Table 6. Torsion angles $\left[{ }^{\circ}\right]$ for $\mathbf{2}^{\bullet+} \mathbf{S b F}_{\mathbf{6}}{ }^{-}$.

\begin{tabular}{|c|c|}
\hline$C(2)-C(3)-C(4)-C(103)$ & $0(4)$ \\
\hline$C(2)-C(3)-C(4)-C(5)$ & $172.4(16)$ \\
\hline $\mathrm{C}(2)-\mathrm{C}(3)-\mathrm{C}(4)-\mathrm{S}(101)$ & $-175(14)$ \\
\hline$C(2)-C(3)-C(4)-S(1)$ & $-2(3)$ \\
\hline $\mathrm{C}(1)-\mathrm{C}(103)-\mathrm{C}(4)-\mathrm{C}(3)$ & $-2(5)$ \\
\hline$C(1)-C(103)-C(4)-C(5)$ & $-173(2)$ \\
\hline $\mathrm{C}(1)-\mathrm{C}(103)-\mathrm{C}(4)-\mathrm{S}(101)$ & $-1(6)$ \\
\hline $\mathrm{C}(1)-\mathrm{C}(103)-\mathrm{C}(4)-\mathrm{S}(1)$ & $168(18)$ \\
\hline $\mathrm{C}(2)-\mathrm{S}(101)-\mathrm{C}(4)-\mathrm{C}(3)$ & $4(11)$ \\
\hline$C(2)-S(101)-C(4)-C(103)$ & $-1(3)$ \\
\hline$C(2)-S(101)-C(4)-C(5)$ & $172.7(10)$ \\
\hline $\mathrm{C}(2)-\mathrm{S}(101)-\mathrm{C}(4)-\mathrm{S}(1)$ & $-4.3(16)$ \\
\hline $\mathrm{C}(1)-\mathrm{S}(1)-\mathrm{C}(4)-\mathrm{C}(3)$ & $2(2)$ \\
\hline$C(1)-S(1)-C(4)-C(103)$ & $-10(14)$ \\
\hline$C(1)-S(1)-C(4)-C(5)$ & $-173.3(10)$ \\
\hline $\mathrm{C}(1)-\mathrm{S}(1)-\mathrm{C}(4)-\mathrm{S}(101)$ & $3.6(13)$ \\
\hline $\mathrm{C}(3)-\mathrm{C}(4)-\mathrm{C}(5)-\mathrm{C}(6)$ & $8(3)$ \\
\hline$C(103)-C(4)-C(5)-C(6)$ & $178(4)$ \\
\hline $\mathrm{S}(101)-\mathrm{C}(4)-\mathrm{C}(5)-\mathrm{C}(6)$ & $4.8(18)$ \\
\hline $\mathrm{S}(1)-\mathrm{C}(4)-\mathrm{C}(5)-\mathrm{C}(6)$ & $-178.1(10)$ \\
\hline $\mathrm{C}(3)-\mathrm{C}(4)-\mathrm{C}(5)-\mathrm{S}(2)$ & $-168(2)$ \\
\hline $\mathrm{C}(103)-\mathrm{C}(4)-\mathrm{C}(5)-\mathrm{S}(2)$ & 2(4) \\
\hline$S(101)-C(4)-C(5)-S(2)$ & $-171.2(11)$ \\
\hline $\mathrm{S}(1)-\mathrm{C}(4)-\mathrm{C}(5)-\mathrm{S}(2)$ & $5.9(13)$ \\
\hline$C(8)-S(2)-C(5)-C(6)$ & $-0.9(9)$ \\
\hline $\mathrm{C}(8)-\mathrm{S}(2)-\mathrm{C}(5)-\mathrm{C}(4)$ & $175.7(9)$ \\
\hline $\mathrm{C}(10)-\mathrm{C}(11)-\mathrm{C}(12)-\mathrm{C}(22)$ & $178.3(11)$ \\
\hline $\mathrm{C}(19)-\mathrm{C}(11)-\mathrm{C}(12)-\mathrm{C}(22)$ & $1.5(16)$ \\
\hline $\mathrm{C}(10)-\mathrm{C}(11)-\mathrm{C}(12)-\mathrm{S}(3)$ & $-1.6(15)$ \\
\hline $\mathrm{C}(19)-\mathrm{C}(11)-\mathrm{C}(12)-\mathrm{S}(3)$ & $-178.5(8)$ \\
\hline $\mathrm{C}(9)-\mathrm{S}(3)-\mathrm{C}(12)-\mathrm{C}(11)$ & $1.9(10)$ \\
\hline$C(9)-S(3)-C(12)-C(22)$ & $-178.0(13)$ \\
\hline $\mathrm{C}(12)-\mathrm{C}(11)-\mathrm{C}(10)-\mathrm{C}(9)$ & $0.3(15)$ \\
\hline$C(19)-C(11)-C(10)-C(9)$ & $176.2(12)$ \\
\hline
\end{tabular}




\begin{tabular}{|c|c|}
\hline $\mathrm{C}(6)-\mathrm{C}(7)-\mathrm{C}(8)-\mathrm{C}(9)$ & $173.2(11)$ \\
\hline$C(6)-C(7)-C(8)-S(2)$ & $-1.5(13)$ \\
\hline $\mathrm{C}(5)-\mathrm{S}(2)-\mathrm{C}(8)-\mathrm{C}(7)$ & $1.4(9)$ \\
\hline $\mathrm{C}(5)-\mathrm{S}(2)-\mathrm{C}(8)-\mathrm{C}(9)$ & $-173.7(10)$ \\
\hline$C(11)-C(12)-C(22)-C(23)$ & $55.2(15)$ \\
\hline$S(3)-C(12)-C(22)-C(23)$ & $-124.9(12)$ \\
\hline$C(11)-C(12)-C(22)-C(24)$ & $-59.9(15)$ \\
\hline $\mathrm{S}(3)-\mathrm{C}(12)-\mathrm{C}(22)-\mathrm{C}(24)$ & $120.0(12)$ \\
\hline$C(12)-C(11)-C(19)-C(20)$ & $-57.5(14)$ \\
\hline$C(10)-C(11)-C(19)-C(20)$ & $126.4(14)$ \\
\hline$C(12)-C(11)-C(19)-C(21)$ & $57.8(14)$ \\
\hline$C(10)-C(11)-C(19)-C(21)$ & $-118.3(15)$ \\
\hline $\mathrm{C}(8)-\mathrm{C}(7)-\mathrm{C}(6)-\mathrm{C}(5)$ & $0.8(15)$ \\
\hline $\mathrm{C}(4)-\mathrm{C}(5)-\mathrm{C}(6)-\mathrm{C}(7)$ & $-176.0(11)$ \\
\hline$S(2)-C(5)-C(6)-C(7)$ & $0.3(12)$ \\
\hline $\mathrm{C}(4)-\mathrm{C}(3)-\mathrm{C}(2)-\mathrm{C}(1)$ & 1(3) \\
\hline$C(4)-C(3)-C(2)-C(16)$ & $-175.3(16)$ \\
\hline$C(4)-C(3)-C(2)-S(101)$ & $176(11)$ \\
\hline$C(4)-S(101)-C(2)-C(1)$ & $3.3(18)$ \\
\hline C(4)-S(101)-C(2)-C(3) & $-3(7)$ \\
\hline $\mathrm{C}(4)-\mathrm{S}(101)-\mathrm{C}(2)-\mathrm{C}(16)$ & $-176.1(11)$ \\
\hline$C(11)-C(19)-C(21)-C(24)$ & $-56.2(14)$ \\
\hline$C(20)-C(19)-C(21)-C(24)$ & $60.2(13)$ \\
\hline$C(1)-C(2)-C(16)-C(18)$ & $-58.7(15)$ \\
\hline $\mathrm{C}(3)-\mathrm{C}(2)-\mathrm{C}(16)-\mathrm{C}(18)$ & $118(3)$ \\
\hline$S(101)-C(2)-C(16)-C(18)$ & $120.8(15)$ \\
\hline$C(1)-C(2)-C(16)-C(17)$ & $57.4(15)$ \\
\hline$C(3)-C(2)-C(16)-C(17)$ & $-126(3)$ \\
\hline$S(101)-C(2)-C(16)-C(17)$ & $-123.2(15)$ \\
\hline$C(11)-C(19)-C(20)-C(23)$ & $54.3(14)$ \\
\hline$C(21)-C(19)-C(20)-C(23)$ & $-60.6(14)$ \\
\hline $\mathrm{C}(2)-\mathrm{C}(16)-\mathrm{C}(17)-\mathrm{C}(14)$ & $-51.9(15)$ \\
\hline$C(18)-C(16)-C(17)-C(14)$ & $63.6(14)$ \\
\hline $\mathrm{C}(7)-\mathrm{C}(8)-\mathrm{C}(9)-\mathrm{C}(10)$ & $-8.6(19)$ \\
\hline$S(2)-C(8)-C(9)-C(10)$ & $165.6(9)$ \\
\hline $\mathrm{C}(7)-\mathrm{C}(8)-\mathrm{C}(9)-\mathrm{S}(3)$ & $179.9(10)$ \\
\hline
\end{tabular}




\begin{tabular}{|c|c|}
\hline$S(2)-C(8)-C(9)-S(3)$ & $-5.9(14)$ \\
\hline$C(11)-C(10)-C(9)-C(8)$ & $-171.1(11)$ \\
\hline $\mathrm{C}(11)-\mathrm{C}(10)-\mathrm{C}(9)-\mathrm{S}(3)$ & $1.1(12)$ \\
\hline $\mathrm{C}(12)-\mathrm{S}(3)-\mathrm{C}(9)-\mathrm{C}(8)$ & $170.9(10)$ \\
\hline$C(12)-S(3)-C(9)-C(10)$ & $-1.7(9)$ \\
\hline$C(3)-C(2)-C(1)-C(103)$ & $-2(3)$ \\
\hline $\mathrm{C}(16)-\mathrm{C}(2)-\mathrm{C}(1)-\mathrm{C}(103)$ & $175(3)$ \\
\hline$S(101)-C(2)-C(1)-C(103)$ & $-4(3)$ \\
\hline $\mathrm{C}(3)-\mathrm{C}(2)-\mathrm{C}(1)-\mathrm{C}(13)$ & $-177.3(19)$ \\
\hline $\mathrm{C}(16)-\mathrm{C}(2)-\mathrm{C}(1)-\mathrm{C}(13)$ & $0.2(17)$ \\
\hline$S(101)-C(2)-C(1)-C(13)$ & $-179.2(14)$ \\
\hline $\mathrm{C}(3)-\mathrm{C}(2)-\mathrm{C}(1)-\mathrm{S}(1)$ & $0(2)$ \\
\hline$C(16)-C(2)-C(1)-S(1)$ & $177.7(10)$ \\
\hline$S(101)-C(2)-C(1)-S(1)$ & $-2(2)$ \\
\hline$C(4)-C(103)-C(1)-C(2)$ & $3(5)$ \\
\hline C(4)-C(103)-C(1)-C(13) & $175.2(19)$ \\
\hline$C(4)-C(103)-C(1)-S(1)$ & $-171(14)$ \\
\hline$C(14)-C(13)-C(1)-C(2)$ & $-57.9(15)$ \\
\hline$C(15)-C(13)-C(1)-C(2)$ & $57.3(15)$ \\
\hline$C(14)-C(13)-C(1)-C(103)$ & $130(5)$ \\
\hline$C(15)-C(13)-C(1)-C(103)$ & $-115(5)$ \\
\hline $\mathrm{C}(14)-\mathrm{C}(13)-\mathrm{C}(1)-\mathrm{S}(1)$ & $124.9(13)$ \\
\hline $\mathrm{C}(15)-\mathrm{C}(13)-\mathrm{C}(1)-\mathrm{S}(1)$ & $-120.0(13)$ \\
\hline$C(4)-S(1)-C(1)-C(2)$ & $-1.1(12)$ \\
\hline C(4)-S(1)-C(1)-C(103) & $6(9)$ \\
\hline $\mathrm{C}(4)-\mathrm{S}(1)-\mathrm{C}(1)-\mathrm{C}(13)$ & $176.0(12)$ \\
\hline$C(12)-C(22)-C(23)-C(20)$ & $-54.1(14)$ \\
\hline $\mathrm{C}(24)-\mathrm{C}(22)-\mathrm{C}(23)-\mathrm{C}(20)$ & $58.5(14)$ \\
\hline$C(19)-C(20)-C(23)-C(22)$ & $1.4(15)$ \\
\hline $\mathrm{C}(12)-\mathrm{C}(22)-\mathrm{C}(24)-\mathrm{C}(21)$ & $56.1(14)$ \\
\hline$C(23)-C(22)-C(24)-C(21)$ & $-58.4(14)$ \\
\hline$C(19)-C(21)-C(24)-C(22)$ & $-0.9(15)$ \\
\hline$C(2)-C(16)-C(18)-C(15)$ & $56.9(15)$ \\
\hline $\mathrm{C}(17)-\mathrm{C}(16)-\mathrm{C}(18)-\mathrm{C}(15)$ & $-59.6(14)$ \\
\hline$C(16)-C(18)-C(15)-C(13)$ & $-2.2(16)$ \\
\hline $\mathrm{C}(1)-\mathrm{C}(13)-\mathrm{C}(15)-\mathrm{C}(18)$ & $-53.6(15)$ \\
\hline
\end{tabular}


C(14)-C(13)-C(15)-C(18)

$59.8(14)$

$\mathrm{C}(16)-\mathrm{C}(17)-\mathrm{C}(14)-\mathrm{C}(13)$

$-4.7(16)$

$\mathrm{C}(1)-\mathrm{C}(13)-\mathrm{C}(14)-\mathrm{C}(17)$

$58.6(14)$

C(15)-C(13)-C(14)-C(17)

$-56.5(14)$

Symmetry transformations used to generate equivalent atoms: 


\section{Calculated Results}

\section{Optimized Strucuture}

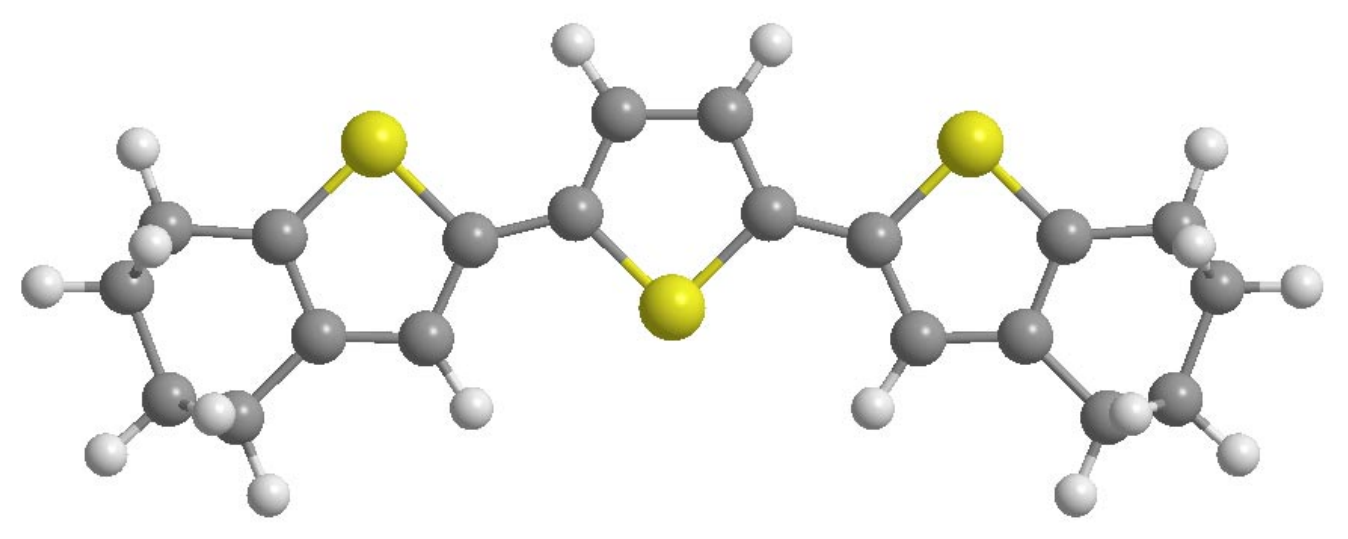

Cartesian coordinates and absolute energies

$\mathbf{2}^{\mathbf{*}}$ (all-anti), $\mathrm{C} 1$, no negative frequencies

B3LYP/6-31G(d) -2123.3519065 hartree

51

$\begin{array}{llll}\mathrm{C} & 6.610503 & 0.604412 & 0.000456\end{array}$

$\begin{array}{llll}\mathrm{C} & 5.121772 & 0.494551 & 0.000123\end{array}$

$\begin{array}{llll}\text { C } & 4.607330 & -0.804161 & -0.000123\end{array}$

$\begin{array}{llll}\mathrm{S} & 3.893547 & 1.698200 & 0.000279\end{array}$

$\begin{array}{llll}\text { C } & 5.680682 & -1.859275 & -0.000365\end{array}$

$\begin{array}{llll}\text { C } & 3.211169 & -0.832531 & -0.000251\end{array}$

$\begin{array}{llll}\text { C } & 2.632499 & 0.448680 & -0.000087\end{array}$

$\mathrm{H} \quad \begin{array}{llll}2.617821 & -1.741554 & -0.000466\end{array}$

$\begin{array}{llll}\text { C } & 1.264795 & 0.796372 & -0.000168\end{array}$

$\begin{array}{llll}\text { C } & 0.692026 & 2.086024 & -0.000181\end{array}$

$\begin{array}{llll}\text { S } & 0.000009 & -0.428566 & -0.000226\end{array}$

$\begin{array}{llll}\text { C } & -0.692024 & 2.086020 & -0.000203\end{array}$

$\begin{array}{llll}\mathrm{H} & 1.291600 & 2.989526 & -0.000135\end{array}$

$\begin{array}{llll}\text { C } & -1.264787 & 0.796365 & -0.000205\end{array}$

$\begin{array}{llll}\mathrm{H} & -1.291603 & 2.989518 & -0.000168\end{array}$

$\begin{array}{llll}\text { C } & -2.632493 & 0.448671 & -0.000124\end{array}$

$\begin{array}{llll}\text { C } & -3.211170 & -0.832538 & 0.000018\end{array}$

$\begin{array}{llll}\mathrm{S} & -3.893540 & 1.698191 & -0.000176\end{array}$ 


\begin{tabular}{lrrc}
$\mathrm{C}$ & -4.607333 & -0.804164 & 0.000069 \\
$\mathrm{H}$ & -2.617824 & -1.741563 & 0.000107 \\
$\mathrm{C}$ & -5.121771 & 0.494550 & -0.000054 \\
$\mathrm{C}$ & -5.680691 & -1.859273 & 0.000072 \\
$\mathrm{C}$ & -6.610503 & 0.604417 & 0.000133 \\
$\mathrm{H}$ & -5.265123 & -2.871172 & 0.000046 \\
$\mathrm{C}$ & -6.565189 & -1.623213 & 1.258216 \\
$\mathrm{C}$ & -6.564973 & -1.623071 & -1.258209 \\
$\mathrm{C}$ & -7.114275 & -0.164773 & 1.260946 \\
$\mathrm{C}$ & -7.114884 & -0.164944 & -1.260313 \\
$\mathrm{H}$ & -6.968138 & 1.637248 & 0.000154 \\
$\mathrm{H}$ & -8.209349 & -0.156934 & 1.243411 \\
$\mathrm{H}$ & -6.805549 & 0.368483 & 2.165237 \\
$\mathrm{H}$ & -6.807366 & 0.368547 & -2.164872 \\
$\mathrm{H}$ & -7.386199 & -2.347483 & -1.249298 \\
$\mathrm{H}$ & -5.980807 & -1.816816 & 2.163200 \\
$\mathrm{H}$ & -7.386832 & -2.347144 & 1.248537 \\
$\mathrm{H}$ & -8.209947 & -0.157747 & -1.241698 \\
$\mathrm{H}$ & -5.980091 & -1.815793 & -2.163063 \\
$\mathrm{H}$ & 5.265108 & -2.871172 & -0.000702 \\
$\mathrm{C}$ & 6.565139 & -1.623588 & 1.257877 \\
$\mathrm{C}$ & 6.565003 & -1.622707 & -1.258548 \\
$\mathrm{H}$ & 6.968141 & 1.637242 & 0.000787 \\
$\mathrm{C}$ & 7.114997 & -0.164612 & -1.260150 \\
$\mathrm{C}$ & 7.114150 & -0.165120 & 1.261110 \\
$\mathrm{H}$ & 6.805264 & 0.367854 & 2.165513 \\
$\mathrm{H}$ & 8.209226 & -0.157216 & 1.243733 \\
$\mathrm{H}$ & 8.210057 & -0.157483 & -1.241383 \\
$\mathrm{H}$ & 5.980754 & -1.817528 & 2.162787 \\
$\mathrm{H}$ & & 0.369162 & -2.164596 \\
$\mathrm{H}$ & -2.386816 & -2.347477 & 1.247963 \\
$\mathrm{H}$ & -1.815087 & -2.163472 \\
$\mathrm{H}$ & -2.347169 & -1.249877 \\
\hline
\end{tabular}




\section{Optimized Strucuture}

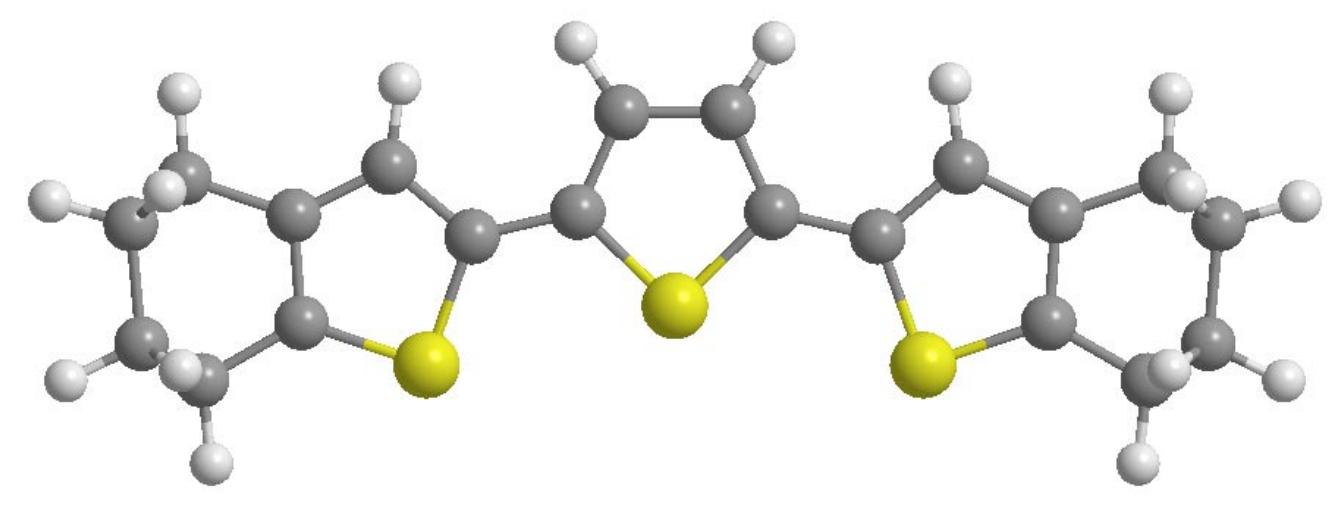

Cartesian coordinates and absolute energies

$\mathbf{2}^{\cdot+}$ (all-syn), C1, no negative frequencies

B3LYP/6-31G(d) -2123.3497717 hartree

51

$\begin{array}{llll}\text { C } & 6.158181 & -1.448264 & -0.000002\end{array}$

$\begin{array}{llll}\text { C } & 4.865244 & -0.702270 & 0.000013\end{array}$

$\begin{array}{llll}\text { C } & 4.964908 & 0.690125 & 0.000034\end{array}$

$\begin{array}{llll}\mathrm{S} & 3.233038 & -1.251112 & -0.000080\end{array}$

$\begin{array}{llll}\mathrm{C} & 6.390236 & 1.175371 & 0.000154\end{array}$

$\begin{array}{llll}\text { C } & 3.720740 & 1.322817 & -0.000012\end{array}$

$\begin{array}{llll}\text { C } & 2.641015 & 0.418185 & -0.000068\end{array}$

$\begin{array}{llll}\mathrm{C} & 1.265233 & 0.737937 & -0.000104\end{array}$

$\begin{array}{llll}\text { C } & 0.691350 & 2.029209 & -0.000123\end{array}$

S $\quad 0.000005 \quad-0.482740 \quad-0.000155$

$\begin{array}{llll}\text { C } & -0.691346 & 2.029207 & -0.000133\end{array}$

$\begin{array}{llll}\text { C } & -1.265227 & 0.737933 & -0.000114\end{array}$

$\begin{array}{llll}\text { C } & -2.641011 & 0.418181 & -0.000093\end{array}$

$\begin{array}{llll}\text { C } & -3.720738 & 1.322814 & -0.000112\end{array}$

$\begin{array}{llll}\mathrm{S} & -3.233039 & -1.251115 & -0.000088\end{array}$

$\begin{array}{llll}\text { C } & -4.964908 & 0.690124 & -0.000019\end{array}$

$\begin{array}{llll}\text { C } & -4.865246 & -0.702271 & 0.000027\end{array}$

$\begin{array}{llll}\text { C } & -6.390236 & 1.175372 & 0.000099\end{array}$

$\begin{array}{llll}\text { C } & -6.158185 & -1.448263 & 0.000070\end{array}$

$\begin{array}{llll}\text { C } & -7.085169 & 0.578909 & -1.257339\end{array}$

$\begin{array}{llll}\text { C } & -7.084860 & 0.578919 & 1.257714\end{array}$ 


$\begin{array}{lrrr}\mathrm{C} & -6.946311 & -0.973356 & -1.260326 \\ \mathrm{C} & -6.946343 & -0.973378 & 1.260453 \\ \mathrm{C} & 7.085149 & 0.578968 & -1.257324 \\ \mathrm{C} & 7.084875 & 0.578856 & 1.257730 \\ \mathrm{C} & 6.946354 & -0.973441 & 1.260395 \\ \mathrm{C} & 6.946290 & -0.973297 & -1.260386 \\ \mathrm{H} & 6.032089 & -2.533923 & -0.000063 \\ \mathrm{H} & 6.454593 & 2.267435 & 0.000212 \\ \mathrm{H} & 3.583900 & 2.398547 & 0.000009 \\ \mathrm{H} & 1.289754 & 2.932198 & -0.000110 \\ \mathrm{H} & -1.289752 & 2.932195 & -0.000125 \\ \mathrm{H} & -3.583897 & 2.398544 & -0.000140 \\ \mathrm{H} & -6.454592 & 2.267436 & 0.000105 \\ \mathrm{H} & -6.032096 & -2.533922 & 0.000058 \\ \mathrm{H} & -6.644062 & 1.007041 & -2.162919 \\ \mathrm{H} & -8.139773 & 0.873950 & -1.246327 \\ \mathrm{H} & -8.139401 & 0.874200 & 1.247158 \\ \mathrm{H} & -6.643287 & 1.006836 & 2.163168 \\ \mathrm{H} & -7.929215 & -1.456334 & -1.242498 \\ \mathrm{H} & -6.436962 & -1.319462 & -2.164899 \\ \mathrm{H} & -6.437215 & -1.319760 & 2.165044 \\ \mathrm{H} & -7.929359 & -1.456123 & 1.242394 \\ \mathrm{H} & 6.644029 & 1.007144 & -2.162875 \\ \mathrm{H} & 8.139754 & 0.874008 & -1.246312 \\ \mathrm{H} & 6.643316 & 1.006729 & 2.163211 \\ \mathrm{H} & 8.139417 & 0.874134 & 1.247173 \\ \mathrm{H} & 7.929368 & -1.456190 & 1.242300 \\ \mathrm{H} & 6.437235 & -1.319866 & 2.164974 \\ & 6.436926 & -1.319358 & -2.164968 \\ \mathrm{H} & 7.929194 & -1.456277 & -1.242598\end{array}$




\section{Explanation for the formation of all-syn conformer of $2^{\circ+} \mathrm{SbF}_{6}{ }^{-}$}

The KS-SOMO of radical cation $\mathbf{2}^{\mathbf{*}}$ is shown in Figure S3. Apparently the MO coefficients on carbon atoms of the $\pi$-systems are much larger than those on sulfur atoms.

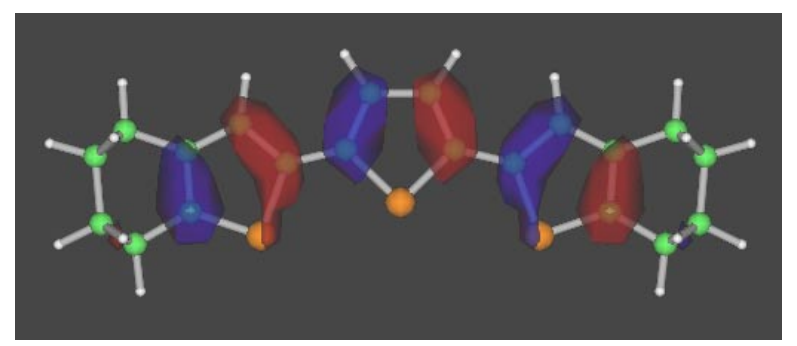

Figure S3. The KS-SOMO of radical cation $\mathbf{2}^{*+}$.

In the case of the all-syn conformer, effective overlaps of SOMOs are possible mainly at the three central areas as shown in Figure S4.

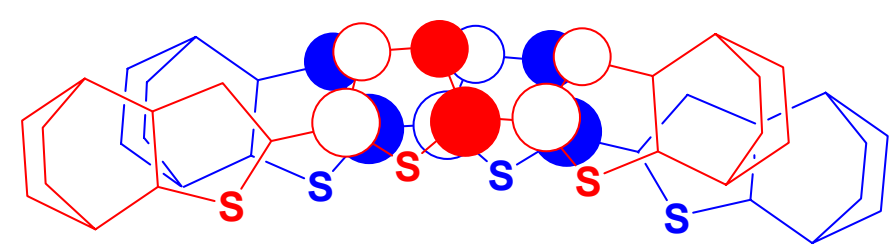

Figure S4. Schematic representation of SOMOs of the central areas of all-syn conformers of the $\pi$-dimer. The phase of the MOs are expressed by filling the circles.

In the case of the all-anti conformer, the area, in which effective overlap of SOMOs is possible, is limited only to the central position as schematically shown in Figure S5.

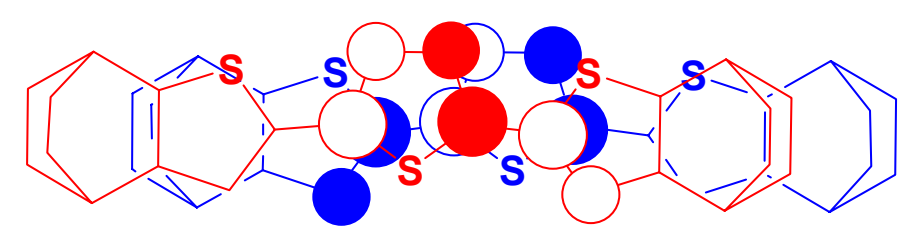

Figure S5. Schematic representation of SOMOs of the central areas of all-anti conformers of the $\pi$-dimer. The phase of the MOs are expressed by filling the circles. 